\title{
ZERO-LOOP OPEN STRINGS IN THE COTANGENT BUNDLE AND MORSE HOMOTOPY*
}

\author{
KENJI FUKAYA ${ }^{\dagger}$ AND YONG-GEUN OH ${ }^{\ddagger}$
}

0. Introduction. Many important works in symplectic geometry and topology are regarded as the symplectization or the quantization of the corresponding results in ordinary geometry and topology. One outstanding example is the celebrated Arnold conjecture which concerns the number of fixed points of a symplectic diffeomorphism or that of intersection points of two Lagrangian submanifolds. The homological version of the conjecture has been proved in various cases (see [Fl1-5], [O2,3,6], [On] and [PSS], and [O7] for a survey and references on the Arnold conjecture and Floer homology). The estimate (in its homological version) predicted by the Arnold conjecture can be regarded as the symplectization or the quantization of the Morse inequality, and conversely the latter can be considered as the semi-classical limit and so a consequence of the former. From now on, we will use the term "quantization" for the similar process that appear below.

To illustrate this statement, we consider the cotangent bundle of a given compact manifold and the graphs of exact one forms. The graph of an exact one form becomes a Lagrangian submanifold of the cotangent bundle with respect to the canonical symplectic structure. Then Floer's result on the Lagrangian intersections $[F 11,3]$ will imply the Morse inequality. The Lagrangian intersection theory is indeed the intersection theoretic version of the Morse theory, while the Lefsechtz intersection theory is that of the degree theory of generic vector fields.

The principle that the symplectic topology and geometry of the cotangent bundle (or more generally that of symplectic manifolds) is the quantization of the ordinary topology and geometry of the base, is a general principle which can be applied to many other situations. The equivalence of the two often holds, when there occurs the absence of the quantum contribution (or the non-existence of the bubbling phenomena). In this paper, we will provide another example of this principle in which we prove that the rational homotopy type of a compact manifold $M$ can be described by the moduli space of pseudo holomorphic disks with appropriate Lagrangian boundary conditions in its cotangent bundle $T^{*} M$. The precise statement of our result is in Section 1.

Our result paves the way to applying the $A^{\infty}$-structure introduced by the first author [Fu2] to the study of the estimate, in terms of the rational homotopy invariant of the base manifold, of the number of intersections of the zero section in the cotangent bundle and its Hamiltonian deformation. This enables us to go one step further, beyond the existing homological estimate in the literature, towards the proof of the original Arnold conjecture which states that the number of the intersections will be greater than or equal to the Morse number of $M$. Viterbo [V] and EliashbergGromov [EG] have also studied this kind of estimate using the generating functions of Lagrangian submanifolds.

* Received January 31, 1997; accepted for publication February 21, 1997.

† Department of Mathematics, Faculty of Sciencess, Kyoto University, Kyoto, Kitashirakawa, JAPAN (fukaya@kusm.kyoto-u.ac.jp). Partially supported by Grant in-Aid for Scientific Research on Priority Areas 231 Infinite Analysis, Grant in-Aid for Scientific Research 07304010 and 07640111 and by Sumitomo foundation.

$\ddagger$ Department of Mathematics, University of Wisconsin, Madison, WI 53706, USA (oh@math. wisc.edu). Partially supported by the NSF Grant DMS-9504455. 
Furthermore analytical details similar to ours in this paper will be required in the various versions of the Floer theory in the symplectic geometry and our proof will also serve there as a cornerstone with obvious but maybe technically tedious modifications. We refer to [PSS] or [RT2] for the announcement of similar analytical results in the context of Hamiltonian diffeomorphisms, and to [08,9] for further applications of the Floer theory to symplectic topology based on such analytic results as one in this paper.

Now we review some of the previous results related to the results in this paper. Floer [Fl1-4] defined and studied Floer homology of the general pair $\left(L_{0}, L_{1}\right)$ of Lagrangian submanifolds on a given symplectic manifold $(P, \omega)$, essentially under the assumption $\pi_{2}(P)=\{e\}$ and $\pi_{1}\left(L_{i}\right)=\{e\}$. Under this assumption, Floer proved that Floer homology is well defined and invariant under the Hamiltonian deformation of $L$ 's. He also proved, under the assumption $\pi_{2}\left(P, L_{0}\right)=\{e\}$, that if $L_{1}$ is a Hamiltonian deformation of $L_{0}$, then a (slightly modified) Floer homology of the intersection of the two Lagrangian submanifolds is the ordinary homology of the Lagrangian submanifold $L_{0}$. Floer's proof (without change) can be applied to the case of the cotangent bundle $P=T^{*} M$ and to the graphs $L$ of exact one-forms, where the assumption $\pi_{2}(P, L)=\{e\}$ is automatically satisfied. Subsequently the second author of the present paper relaxed Floer's assumption and developed the Floer theory for the class of monotone Lagrangian submanifolds, which includes Floer's as a special case (See $[02,3,6])$. One difference of the general monotone case from Floer's is the existence of non-trivial quantum contribution which changes the Floer homology from the ordinary homology. We refer to [06] for some application of the study of the quantum contribution to the symplectic topology of Lagrangian embeddings.

In the mean time, inspired by a talk by Donaldson [D], the first author further studied Lagrangian intersections and pseudo holomorphic curves where there are involved 3 Lagrangian submanifolds or more (this problem is also related to the study of the (gauge theory) Floer homology of 3-manifolds with boundary as was discussed in $[$ Fu1 12,4$])$, and discovered an $A^{\infty}$ structure on the Floer homology. $A^{\infty}$ structure was first discovered by Stasheff in the study of homotopy theoretic structures in the algebraic topology ([St1]).

As is discussed in [Fu2,3], the construction of the $A^{\infty}$ structure on the Floer homology is parallel to that of quantum ring discussed in [R], [RT1] and [KM]: Roughly speaking, the $A^{\infty}$ structure on the Floer homology is the 0-loop correlation function of the (topological) open string while the quantum ring (and the quantum higher Massey product defined in [Fu3]) is the 0-loop correlation function of the (topological) closed string. Similar $A^{\infty}$ structures are discovered independently by various physicists in the context of the string theory and also by M. Kontsevitch [Ko1,2]. The operad structure discussed by various mathematicians (see [Ge], [St2], [HL]) is that corresponding to our $A^{\infty}$ structure in the closed string.

The first author next applied the same construction of this $A^{\infty}$ structure in the context of the Morse theory. The basic idea is to use several Morse functions simultaneously and to study the corresponding ordinary differential equations given by the gradient vector fields on arbitrary trees, which will produce an $A^{\infty}$ structure on the ordinary homology group (more precisely on the Morse homology). We call these constructions the Morse homotopy theory. The idea of using several Morse functions simultaneously to deduce more information of the topology of manifolds was independently discovered also by M. Betz and R. Cohen [BC]. It turns out that this $A^{\infty}$ structure thus constructed on the (co)homology group (in the case of rational coefficient) is the Morse homotopy analogue of the De-Rham homotopy theory of 
D.Sullivan [Su]. Therefore by the result of D. Sullivan [Su] and D.Quillen [Q], it follows that $A^{\infty}$ structure determines the rational homotopy type of the manifold.

The main goal of this paper is to show that the Morse homotopy theory on a manifold $M$ which uses trees as the graphs is equivalent to the open string theory of 0-loop on its cotangent bundle. In mathematical language, the topological open string theory of 0 -loop means the study of pseudo holomorphic disks with Lagrangian boundary condition. Therefore our main result (Theorem 1.7) implies that the rational homotopy type of a manifold can be described also by pseudo holomorphic disks in its cotangent bundle.

We would like to mention here some more results which are relevant to the present paper. In [W2], Witten discussed a relation of the 0-loop open string theory to noncommutative geometry of $\mathrm{A}$. Connes [Co] and hinted for example, that coefficients of the q-th composition map in the $A^{\infty}$ structure on Floer homology has a cyclic symmetry which can be related to the theory of cyclic cohomology in the noncommutative geometry. Compare this also with Kontsevitch's paper [Ko1]. Subsequently, Witten expanded this point of view to include the higher loop case in [W4], namely the case of Riemann surfaces of higher genus, and discovered that the Chern-Simons perturbation theory developed in [AS], [Ba], [GMM] and [Ko1] can be described by the higher genus correlation function of open strings on the cotangent bundle. Our point of view that the open string theory is the quantization of the Morse theory can be also applied to the case of general Riemann surfaces: The higher loop correlation functions in the topological open string theory on the cotangent bundle are the quantization of the Morse homotopy of general graphs of higher loop on the base manifold. We refer to [Fu6], especially Section 8, in regard to this point of view. A more systematic study of open strings of higher loop is the subject of future research.

The organization of this paper is as follows. In Section 1, we give the definitions of the two moduli spaces, one that of graph flows in the Morse theory and the other that of pseudo-holomorphic discs in the symplectic geometry, and state our main result which asserts their equivalence. In Section 2, we give a brief summary of the $A^{\infty}$ structure and explain what our main result means to the $A^{\infty}$ structure. Sections 3 to 17 of the paper are devoted to the proof of the main theorem. Those sections are divided into two parts.

Part $\mathrm{I}$ is devoted to the case in which we concern three Lagrangians and three Morse functions. In this case, our main theorem asserts that studying the zerodimensional part of the moduli-space of pseudo holomorphic disks with the corresponding Lagrangian boundary condition gives rise to the cup product of the base manifold. Part I is mainly of the analytic nature. The similar analytic argument will be required in Part II where the general case is studied. In Part II, we will not repeat those analytic details we provide in Part I, but focus only on the new phenomena we need to handle with. The contents of each sections of Part I and II are in order. In Section 3, we provide the appropriate analytical set up of the Sobolev space we use, and re-state the main theorem in the case of the three Lagrangians. In Section 4, to each given element of the moduli space of the graph flows, we explicitly construct a map from a disk to the cotangent bundle which is approximately (pseudo)-holomorphic. Section 5 is devoted to the error estimate of these approximate solutions. In Section 6 , we prove that the linearized operators of the approximate solutions are surjective, when the moduli space of the graph flow in the Morse theory satisfy appropriate transversality condition. The (Fredholm) inverse of this linearized equation is studied in Section 7 where we establish various estimates we need later. Using the estimates 
in Section 8, we find an exact solution in a neighborhood of the approximate solution defined in Section 3. In Section 9, we prove that every pseudo holomorphic disk in our moduli space is obtained in this way (in the semi-classical limit). This completes the proof of the main theorem in the case of three Lagrangians.

One main new phenomenon we must take care of in the general case is that the domains of the equations have moduli themselves. In the case of pseudo holomorphic disks, the space of conformal structures on the disc with $k$ marked points on its boundary has moduli when the number of marked points are bigger than 3 , while the conformal structure is unique if the number of point is 3 or less. Similarly in the case of the Morse theory, we need to consider the moduli space of metrics on the corresponding trees. Therefore to compare the moduli space of pseudo holomorphic disks with that of graph flows, we also need to incorporate these moduli of the conformal structures on the disk with $k$ marked points on its boundary and of the metric structures of the relevant graphs.

In Section 10, we study the stratification of the moduli space of metric structures of the graph. This stratification also induces the corresponding stratification on the moduli space of the graph flow. In Section 11, we construct approximate solutions of the pseudo holomorphic curve equation in a way similar to Section 4, where we need to work on each of the strata separately. Because of the moduli of the domains, the construction of approximate solutions is more delicate than in Section 3. In Section 12 and 13, we construct exact solutions of pseudo holomorphic curve equation out of these approximate solutions on each stratum. The main point we need to discuss at this stage is to prove that the linearized operators at the approximate solutions are surjective. Again this will follow from an appropriate transversality condition of the moduli space of the graph flows. This transversality is carefully discussed in Section 12 and exact solutions of the pseudo holomorphic curve equation are constructed in Section 13.

By now, we have found diffeomorphisms between the two moduli spaces in each stratum. Section 14, 15, 16 and 17 are devoted to the proof that these diffeomorphisms can be glued to construct a global diffeomorphism between the two moduli spaces of pseudo holomorphic disks and of graph flows. We do this in three steps. First, we provide an identification of the moduli space of conformal structures on the disc with $k$ marked points on its boundary and that of metric structures on the trees (with $k$ exterior edges). Stasheff proved in [St1] that the latter is homeomorphic to the Euclidean space. We re-prove his theorem and also show that the natural cell decompositions in the two moduli spaces are dual to each other under the above identification. This argument involves the theory of quadratic differentials and the triangulation of the moduli space of marked Riemann surface (See [Mu], [Ha], [P], [Str] for some explanations on these subjects. Our case is the real version of those in the literature). This result, in particular, implies that the moduli space of metric structures on the graphs is a manifold. Using this, we define a smooth structure on the moduli space of graph flows in Section 15. Finally in Section 16 and 17, we complete the proof of the main theorem.

Both authors would like to thank Newton Institute for its hospitality, where they both stayed and where the present work was initiated. They would also like to thank K. Ono for some helpful discussions.

1. Statement of the main results. In this section, we define two moduli spaces of our concern, one in the Morse theory and the other in the symplectic geometry. To describe the Morse theory side, we first introduce the moduli space of metric Ribbon 
trees.

Definition 1.1. A ribbon tree is a pair $(T, i)$ such that $T$ is a tree and $i: T \rightarrow$ $D^{2} \subset \mathbb{C}$ is an embedding which satisfy the following :

(1.1.1) No vertex of $T$ has 2-edges.

(1.1.2) If $v \in T$ is a vertex with one edge, then $i(v) \in \partial D^{2}$.

(1.1.3) $i(T) \cap \partial D^{2}$ consists of vertices with one edge.

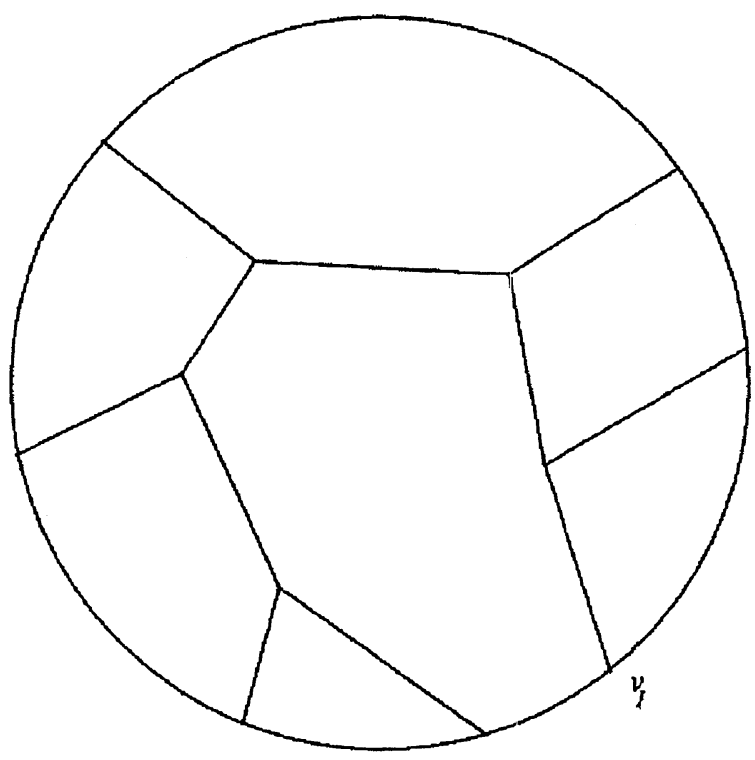

Figure 1.1

We identify two pairs $(T, i)$ and $\left(T^{\prime}, i^{\prime}\right)$, if $T$ and $T^{\prime}$ are isomorphic and $i$ and $i^{\prime}$ are isotopic. Let $G_{k}$ be the set of all triples $\left(T, i, v_{1}\right)$ where $(T, i)$ is as above, $v_{1} \in T \cap \partial D^{2}$ and $T \cap \partial D^{2}$ consists of $k$ points.

We remark that choosing $v_{1} \in T \cap \partial D^{2}$ is equivalent to choosing an order of $T \cap \partial D^{2}$ which is compatible with the cyclic order of $\partial D^{2}=S^{1}$.

DEFINITION 1.2. We call a vertex an interior vertex if it has more than 2 edges attached to it and call it an exterior vertex otherwise. We call an edge an interior edge if both of its vertices are interior and call it exterior otherwise. Let $C_{e x t}^{1}(T)$ be the set of all exterior edges and $C_{i n t}^{1}(T)$ be the set of all interior edges. $C_{e x t}^{0}(T)$ and $C_{\text {int }}^{0}(T)$ stand for the set of exterior and interior vertices respectively.

For each $\mathfrak{t}=\left(T, i, v_{1}\right) \in G_{k}$, let $G r(\mathfrak{t})$ be the set of all maps $\ell: C_{i n t}^{1}(T) \rightarrow \mathbb{R}^{+}$. We put $G r_{k}=\bigcup_{t \in G_{k}} G r(\mathfrak{t})$ and define a topology on it as follows:

Let $\ell_{i} \in G r(\mathfrak{t})$. We assume that $\lim _{i \rightarrow \infty} \ell_{i}(e)$ converges to $\ell_{\infty}(e)$ for all $e \in C_{i n t}^{1}(T)$. Let $\mathfrak{t}^{\prime}=\left(T^{\prime}, i^{\prime}, v_{1}\right) \in G_{k}$ be the ribbon tree obtained by collapsing all the edges $e$ in $T$ such that $\ell_{\infty}(e)=0$. We define $\bar{\ell}_{\infty}: C_{i n t}^{1}\left(T^{\prime}\right) \rightarrow \mathbb{R}^{+}$by the restriction of $\ell_{\infty}$. We then say that the limit of $\ell_{i} \in G r(t)$ is $\ell_{\infty}$. From the definition, it is easy to see that $G r_{k}=\bigcup_{t \in G_{k}} G r(\mathfrak{t})$ provides a cell decomposition of $G r_{k}$. Stasheff [St1] proved that $G r_{k}$ is homeomorphic to $\mathbb{R}^{k-3}$. We give an alternative proof of this statement later 
in Section 14, where we also explicitly provide a smooth structure on $G r_{k}$.

We next introduce the moduli space $\mathfrak{T}_{0, k}$ of disks with $k$ marked points on the boundary as follows: We define

$$
\mathfrak{T}_{0, k}=\frac{\left\{\begin{array}{l|l}
\left(z_{1}, \cdots, z_{k}\right) \in\left(\partial D^{2}\right)^{k} & \begin{array}{l}
z_{i} \neq z_{j}, \quad \text { if } i \neq j \\
z_{1}, \cdots, z_{k} \text { respects the cyclic order of } \partial D^{2}
\end{array}
\end{array}\right.}{\sim}
$$

We use the counter clockwise cyclic ordering for $S^{1}=\partial D^{2}$. Here $\left(z_{1}, \cdots, z_{k}\right) \sim$ $\left(z_{1}^{\prime}, \cdots, z_{k}^{\prime}\right)$ if and only if there exists a biholomorphic map $\varphi: D^{2} \rightarrow D^{2}$ such that $\varphi\left(z_{i}\right)=z_{i}^{\prime}$.

LEMMA 1.3. $\mathfrak{T}_{0, k}$ is homeomorphic to $\mathbb{R}^{k-3}$.

Proof. It is well known that there exists a unique bi-holomorphic map $\varphi: D^{2} \rightarrow$ $D^{2}$ such that $\varphi\left(z_{1}\right)=1, \varphi\left(z_{2}\right)=\sqrt{-1}, \varphi\left(z_{3}\right)=-1$. Hence we have

$$
\mathfrak{T}_{0, k}=\left\{\begin{array}{l|l}
\left(z_{4}, \cdots, z_{k}\right) \in\left(\partial D^{2}\right)^{k-3} & \begin{array}{l}
z_{i} \neq z_{j}, \quad \text { if } i \neq j \\
\operatorname{Im} z_{i}<0, \\
\operatorname{Re} z_{i+1}>\operatorname{Re} z_{i}
\end{array}
\end{array}\right\}
$$

Then the map : $\mathfrak{T}_{0, k} \rightarrow \mathfrak{T}_{0, k-1}\left(z_{4}, \cdots, z_{k}\right) \mapsto\left(z_{4}, \cdots, z_{k-1}\right)$ is a fiber bundle and its fiber is homeomorphic to $\mathbb{R}$. Lemma 1.3 then immediately follows.

Our main result of this paper identifies two moduli spaces, one is related to Morse theory, and the other is related to symplectic geometry more specifically to the Lagrangian intersection theory. We next define those moduli spaces.

Let $f_{1}, \cdots, f_{k}$ be $C^{\infty}$-functions on $M$, and $g$ be a Riemannian metric on $M$. We assume that $f_{i+1}-f_{i}$ is a Morse function for each $i$. (Here we put $f_{k+1}=f_{1}$.) An element of $\mathcal{M}_{g}(M: \vec{f}, \vec{p})$ is a pair $\left(\left(T, i, v_{1}, \ell\right), I\right)$ of elements of $\left(T, i, v_{1}, \ell\right) \in G r_{k}$ and a map $I: T \rightarrow M$ satisfying the Conditions (1.2.1), (1.2.2), (1.2.3) below.

(1.2.1) $I$ is continuous, $I\left(v_{i}\right)=p_{i}$.

Before stating two other conditions, let us fix some notations. The set $D^{2}-i(T)$ has $k$ connected components. We denote them by $D_{i}$ where the closure $\bar{D}_{i}$ contains $v_{i}$ and $v_{i+1}$. We define a metric on $T$ such that the exterior edge is isometric to $(-\infty, 0]$ and the interior edge $e$ is isometric to $[0, \ell(e)]$.

For each $e \in C_{\text {int }}^{1}(T)$ we fix its orientation with respect to which the 2 vertices $i(e)$ and $o(e)$ are determined so that $e$ goes from $i(e)$ to $o(e)$. Note that for each given edge $e$ there are two of the domains $D_{i}$ such that its closure contains $e$. We define the integers $l e f(e)$ and rig(e) so that the closure of $D_{l e f(e)}$ contains $e$ and $D_{l e f(e)}$ is on the left side of $e$ with respect to the orientation of $e$ and $\mathbb{R}^{2}$. The definition of $r i g(e)$ is similar (Figure 1.2). There are $k$ exterior vertices. Let $e_{i}$ 's be the exterior edges containing $v_{i}$. Then we may set $\operatorname{le} f\left(e_{i}\right)=i+1, \operatorname{rig}\left(e_{i}\right)=i$.

Now two other conditions for $\left(\left(T, i, v_{1}, \ell\right), I\right)$ to be an element of $\mathcal{M}_{g}(M: \vec{f}, \vec{p})$ are given as follows:

(1.2.2) Let $e_{i} \in C_{e x t}^{1}$ and identify $e_{i} \simeq(-\infty, 0]$. Then

$$
\frac{\left.d I\right|_{e_{i}}}{d t}=-\operatorname{grad}\left(f_{i+1}-f_{i}\right)
$$

(1.2.3) Let $e \in C_{\text {int }}^{1}$ and identify $e \simeq[0, \ell(e)]$. Then

$$
\frac{\left.d I\right|_{e_{i}}}{d t}=-\operatorname{grad}\left(f_{l e f(e)}-f_{\text {rig(e) }}\right) \text {. }
$$




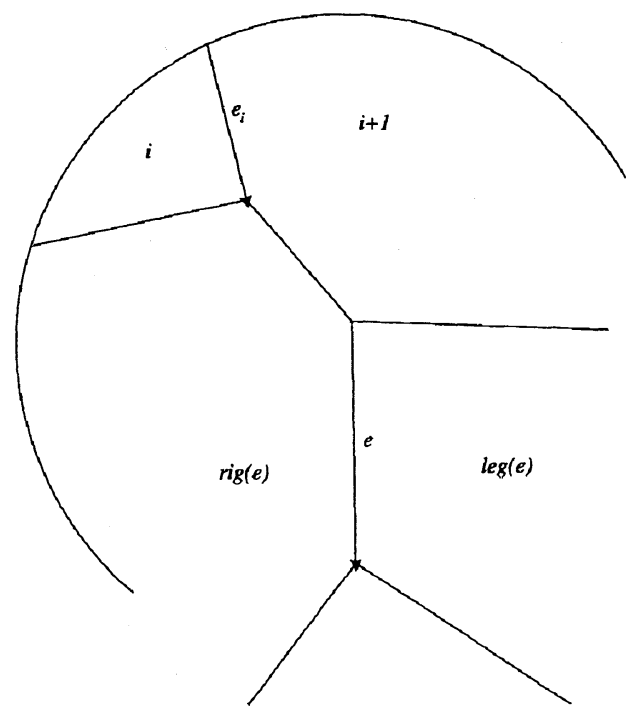

Figure 1.2

We have a natural projection

$$
\pi: \mathcal{M}_{g}(M: \vec{f}, \vec{p}) \rightarrow G r_{k} .
$$

TheOREM 1.4. For generic $f_{1}, \cdots, f_{k}$, the space $\mathcal{M}_{g}(M: \vec{f}, \vec{p})$ is a $C^{\infty}$-manifold of dimension

$$
\sum_{i=1}^{k} \mu\left(p_{i}\right)-(k-1) n+(k-3)
$$

such that $\pi$ becomes a smooth map where $n=\operatorname{dim} M$.

Here $\mu\left(p_{i}\right)=\mu_{\left(f_{i+1}-f_{i}\right)}\left(p_{i}\right)$ is the Morse index of the critical point $p_{i}$ of $f_{i+1}-f_{i}$ for $i=1, \cdots n(\bmod n)$. This theorem was stated without proof in [Fu2, 3,5]. We will prove it in $\S 15$.

We next define another moduli space $\mathcal{M}_{J}\left(T^{*} M: \vec{\Lambda}^{\epsilon}, \vec{p}^{*}\right)$ in the symplectic geometry side. We let $\Lambda_{i}^{\epsilon}$ be the graph of $\epsilon d f_{i} \subset T^{*} M$. This is a Lagrangian submanifold. For each critical point $p$ of $f_{i}-f_{j}$, we can associate a point $x^{\epsilon}$ in the intersection $\Lambda_{i}^{\epsilon} \cap \Lambda_{j}^{\epsilon}$. Namely for a critical point $p_{i}$ of $f_{i+1}-f_{i}$, we put $x_{i}^{\epsilon}=\left(p_{i}, \epsilon d f_{i}\left(p_{i}\right)\right)$ which is a point in the intersection $\Lambda_{i}^{\epsilon} \cap \Lambda_{i+1}^{\epsilon}$.

We now take an almost complex structure $J$ that is compatible to the standard symplectic form $\omega$ on $T^{*} M$ and define

Definition 1.5. The moduli space $\mathcal{M}_{J}\left(T^{*} M: \vec{\Lambda}^{\epsilon}, \vec{x}^{\epsilon}\right)$ consists of the pairs $\left(\left[z_{1}, \cdots, z_{k}\right], \omega\right)$ of elements $\left(\left[z_{1}, \cdots, z_{k}\right] \in \mathfrak{T}_{0, k}\right.$ and a map $\omega: D^{2} \rightarrow T^{*} X$ satisfying the following conditions (1.3.1), (1.3.2) and (1.3.3) (We remark that $\partial D^{2}-\left\{z_{1}, \cdots, z_{k}\right\}$ consists of $k$ connected components.): Let $\partial_{i} D^{2}$ be the component whose closure contains $z_{i}$ and $z_{i+1}$.

(1.3.1) $w\left(z_{i}\right)=p_{i}^{\epsilon}$.

(1.3.2) $w\left(\partial_{i}\left(D^{2}\right)\right) \subset \Lambda_{i}^{\epsilon}$.

(1.3.3) $J \circ T w=T w \circ J$. 
Again there is a natural map

$$
\mathcal{M}_{J}\left(T^{*} M: \vec{\Lambda}^{\epsilon}, \vec{x}^{\epsilon}\right) \rightarrow \mathfrak{T}_{0, k}
$$

Theorem 1.6. For generic $f_{i}$, the space $\mathcal{M}_{J}\left(T^{*} M: \vec{\Lambda}^{\epsilon}, \vec{x}^{\epsilon}\right)$ is a $C^{\infty}$ manifold of dimension

$$
\sum_{i=1}^{k} \mu\left(p_{i}\right)-(k-1) n+(k-3)
$$

where $\mu\left(p_{i}\right)$ are the same integers as in Theorem 1.4.

The proof of this theorem in general involves a transversality argument under the perturbation of boundary conditions rather than under the perturbation of almost complex structures, and some index calculation. See $[04,5]$ for this transversality argument for $k=0$ case and [O9] for an index calculation relevant to the dimension formula in this theorem. We will not give the complete proof of Theorem 1.6 here, because in the case of our main theorem in which $\epsilon$ will be assumed to be sufficiently small, we can prove it in a different way (during the proof of main theorem.)

We now restrict ourselves to the canonical almost complex structure $J=J_{g}$ on $T^{*} M$ that is naturally induced from the Levi-Civita connection of the metric $g$ on $M$. From now on, we will always assume, unless otherwise specified, that $J$ is this canonical almost complex structure. We first note that if a Riemannian metric $g$ is given to $M$, the associated Levi-Civita connection induces a natural almost complex structure on $T^{*} M$, which we denote by $J_{g}$ and which we call the canonical almost complex structure (in terms of the metric $g$ on $M$ ). We are going to fix the Riemannian metric $g$ on $M$ once and for all. This canonical almost complex structure has the following properties:

(1.4.1) $J_{g}$ is compatible with the canonical symplectic structure $w$ on $T^{*} M$.

(1.4.2) For every $(q, p) \in T^{*} M, J_{g}$ maps the vertical tangent vectors to horizontal vectors with respect to the Levi-Civita connection of $g$.

(1.4.3) On the zero section $o_{M} \subset T^{*} M, J_{g}$ assigns to each $v \in T_{q} M \subset T_{(q, 0)}\left(T^{*} M\right)$ the cotangent vector $J_{g}(v)=g(v, \cdot) \in T_{q}^{*} M \subset T_{(q, 0)}\left(T^{*} M\right)$. Here we use the canonical splitting

$$
T_{(q, 0)}\left(T^{*} M\right)=T_{q} M \oplus T_{q}^{*} M .
$$

Now we are ready to state our main theorem.

THEOREM 1.7. Let $J=J_{g}$ be the canonical almost complex structure on $T^{*} M$ associated to the metric $g$ on $M$. For each generic $\vec{f}=\left(f_{i}\right)$ and for sufficiently small $\epsilon$, we have an oriented diffeomorphism $\mathcal{M}_{g}(M: \vec{f}, \vec{p}) \simeq \mathcal{M}_{J}\left(T^{*} M: \vec{\Lambda}^{\epsilon}, \vec{x}^{\epsilon}\right)$.

2. $A^{\infty}$-structures. Here we briefly discuss the definition of $\mathrm{A}^{\infty}$-category and show that our main theorem provides an isomorphism between two $\mathrm{A}^{\infty}$-categories, one in the Morse theory and the other in the Lagrangian intersection theory. We refer to [Fu2, 3, 5] for more details on $\mathrm{A}^{\infty}$-categories.

Definition 2.1. An $\mathrm{A}^{\infty}$-category $\mathfrak{C}$ consists of a set $\mathfrak{O} \mathfrak{B}$ the set of objects and a cochain complex $C^{*}(a, b)$ for each $a, b \in \mathfrak{O} \mathfrak{B}$ (that is the set of morphisms) and a map

$$
q^{k}: C^{*}\left(c_{0}, c_{1}\right) \otimes \cdots \otimes C^{*}\left(c_{k-1}, c_{k}\right) \rightarrow C^{*}\left(c_{0}, c_{k}\right)
$$

of degree $-(k-2)$ such that

$$
\left(d q^{k}-(-1)^{k} q^{k} d\right)\left(x_{1} \otimes \cdots \otimes x_{k}\right)=\sum_{i, j}(-1)^{j} q^{k-j}\left(x_{1} \otimes \cdots q^{j}\left(x_{i} \otimes \cdots \otimes x_{i+j}\right) \cdots \otimes x_{k}\right)
$$


In the case in which there is only one object, the $\mathrm{A}^{\infty}$-category is called an $\mathrm{A}^{\infty}$-algebra (This notion was introduced by Stasheff [St1].)

Our moduli spaces defined in Section 1 can be used to define $\mathrm{A}^{\infty}$-categories. More precisely, we will define topological $\mathrm{A}^{\infty}$-categories as follows.

DefinItion 2.2. A topological $\mathrm{A}^{\infty}$-category consists of a topological space $\mathfrak{O} \mathfrak{B}$ and a chain complex $C(a, b)$ for each pair $a, b$ in a Baire subset of $\mathfrak{O}^{2}$. We assume that they satisfy the properties in Definition 2.1 if $\left(c_{1}, \cdots, c_{k}\right)$ is contained in a Baire subset of $\mathfrak{O B B}^{k}$. We also assume that $q^{k}$ is locally constant with respect to $c_{i}$ where it is defined.

We first consider the case of the Morse theory and define an $A^{\infty}$ category $\mathfrak{M S}(M)$ for each Riemannian manifold $M$. Our object in this case is the set of all smooth functions $C^{\infty}(M)$. For almost all pairs $f, g \in C^{\infty}(M)$, the difference $f-g$ is a Morse function and its gradient flow is a Morse-Smale flow. Hence we can define its Morse-Witten complex $C_{*}(M: f-g)$. Recall that the group $C_{k}(M: h)$ is defined by

$$
C_{k}(M: h)=\text { the free abelian group genererated by } \mathrm{Crit}_{k}(h)
$$

where $\mathrm{Crit}_{k}(h)$ is the set of critical points of the Morse index $k$ (See [Mi], [Fl3], [W1] or [Sc] for more details). Let us then define the dual complex

$$
C^{k}(f, g)=\operatorname{Hom}\left(C_{k}(M: f-g), \mathbb{Z}\right) .
$$

Note that this dual complex can be canonically identified with $C_{\operatorname{dim} M-k}(M:-(f-$ $g))=C_{\operatorname{dim} M-k}(M, g-f)$ and so we will take

$$
C^{k}(f, g)=C_{\operatorname{dim} M-k}(M: g-f)
$$

as the definition in this paper.

Now our $k$-th composition map $q^{k}$ is defined as follows: For each $p_{i} \in \operatorname{Crit}\left(M, f_{i+1}\right.$ $\left.-f_{i}\right)$ for $i=1, \cdots, k+1(\bmod n=\operatorname{dim} M)$, we count the number of zero dimensional components (which can be shown to be compact (and so finite) later). We denote this number by $\sharp \mathcal{M}_{g}(M: \vec{f}, \vec{p})$. In terms of the definition

$$
C^{*}\left(f_{i}, f_{i+1}\right)=C_{n-*}\left(M: f_{i+1}-f_{i}\right),
$$

$p_{i}$ has degree $n-\mu_{\left(f_{i+1}-f_{i}\right)}\left(p_{i}\right)$ for $i=1, \cdots, k$ and $p_{k+1} \in C_{*}\left(f_{1}, f_{k}\right)=C_{*}\left(f_{k+1}, f_{k}\right)$ has degree $\mu_{\left(f_{k+1}-f_{k}\right)}\left(p_{k+1}\right)$. Therefore from the dimension formula in Theorem 1.4 which can be re-written for $\left(f_{1}, \cdots, f_{k+1}\right)$ as

$$
\operatorname{dim} \mathcal{M}_{g}(M: \vec{f}, \vec{p})=\mu\left(p_{k+1}\right)-\sum_{j=1}^{k}\left(n-\mu\left(p_{i}\right)\right)+(k-2),
$$

we derive that $\operatorname{dim} \mathcal{M}_{g}(M: \vec{f}, \vec{p})=0$ when

$$
\operatorname{deg}\left(p_{k+1}\right)=\sum_{j=1}^{k} \operatorname{deg}\left(p_{j}\right)-(k-2) .
$$

Now we define our $k$-th composition map $q^{k}$ by

$$
q^{k}\left(\left[p_{1}\right] \otimes \cdots \otimes\left[p_{k}\right]\right)=\sum \sharp \mathcal{M}_{g}(M: \vec{f}, \vec{p})\left[p_{k+1}\right]
$$

where the sum is taken over all $\left(p_{1}, \cdots, p_{k+1}\right)$ satisfying (2.2). Using Theorem 1.4 and the description of compactification of $\mathcal{M}_{g}(M: \vec{f}, \vec{p})$, one can prove by the stan- 
dard compactness and cobordism arguments that they satisfy the axioms of the $A^{\infty}$ category in Definition 2.1 (See [Fu2] for some details.)

We next discuss the $A^{\infty}$ category in Lagrangian intersection theory. The construction is based on the Floer homology of Lagrangian intersections. There is some difficulty in defining the Floer homology for general Lagrangian submanifolds in general symplectic manifolds as pointed out by the second author [O2] (even if we assume that the symplectic manifold is semi positive), which requires various restrictions on the Lagrangian submanifolds. To avoid such trouble in this paper, we consider only the case in which the Lagrangians are the graphs of exact one forms in the cotangent bundle, which is relevant to our main theorem. As we mentioned in the introduction, the construction of the Floer homology is well-defined in this case. Now the definition of the $A^{\infty}$ category $\mathfrak{S} \mathfrak{Y}\left(T^{*} M\right)_{0}$ is as follows:

Its objects are graphs $\Lambda_{f}$ of exact one forms $d f$. For two objects $\Lambda_{f}, \Lambda_{g}$, we define the morphisms

$$
C^{*}\left(\Lambda_{f}, \Lambda_{g}\right)=C F^{*}\left(\Lambda_{f}, \Lambda_{g}\right)
$$

where $C F^{*}\left(\Lambda_{f}, \Lambda_{g}\right)$ is Floer's cochain complex with an appropriate grading.

Recall that as an abelian group $C F^{*}\left(\Lambda_{f}, \Lambda_{g}\right)$ can be identified with $C F_{n-*}\left(\Lambda_{g}\right.$, $\Lambda_{f}$ ) (by a chain isomorphism) that is generated by the intersections of the two Lagrangians $\Lambda_{f_{1}}$ and $\Lambda_{f_{2}}$. Now we are ready to define the (higher) composition $q^{k}$. Let $\left.x_{i} \in \Lambda_{i} \cap \Lambda_{i+1}\right)$ regarded as an element in $C^{*}\left(\Lambda_{i}, \Lambda_{i+1}\right)$. We define similarly as in the case of Morse theory

$$
q^{k}\left(\left[x_{1}\right] \otimes \cdots \otimes\left[x_{k}\right]\right)=\sum \sharp \mathcal{M}_{J}(M: \vec{f}, \vec{p})\left[x_{k+1}\right] .
$$

Again the sum is taken over those $x_{j}=\left(p_{j}, d f_{j}\left(p_{j}\right)\right)$ 's where the $p_{j}$ 's satisfy (2.2), which will imply that $\mathcal{M}_{J}(M: \vec{f}, \vec{x})$ is 0 -dimensional. Furthermore by the same kind of degree counting as in the case of Morse theory, it follows that $q^{k}$ has degree $-(k-2)$ if we use the grading on $C^{*}\left(\Lambda_{f}, \Lambda_{g}\right)$ transfered from the Morse grading above.

To establish that the map $q^{k}$ is really well-defined and satisfies the axioms in Definition 2.2 with $\mathbb{Z}$-coefficients in general, we need to prove a more general version of the index formula than in Theorem 1.6, which will replace the Morse index $\mu\left(p_{i}\right)$ by the Maslov-type index of the Lagrangian intersections, and to study coherent orientations and compactification of the moduli space. This itself should constitute a nontrivial amount of work and so we will just use our main theorem to transfer here the corresponding results in the Morse theory (which is much easier to prove) for the case in which $f_{i}$ 's are sufficiently small. We will refer elsewhere for the complete proof of the fact that $q^{k}$ 's satisfy all the axioms of the $A^{\infty}$ category.

At least, we can state here the following result which is an immediate translation of our main theorem.

THEOREM 2.3. $\mathfrak{M S}(M)$ is isomorphic to a sub-category of $\mathfrak{S Y}\left(T^{*} M\right)_{0}$.

Remark 2.4. Although we call $\mathfrak{S Y}\left(T^{*} M\right)_{0}$ and $\mathfrak{M S}(M) A^{\infty}$ categories, they are in fact very close to $A^{\infty}$ algebras. This is because there exist canonical isomorphisms between the objects in the above $A^{\infty}$-categories.

\section{PART I: CUP PRODUCT}

3. Preliminaries. In this Part I, we will consider $\mathcal{M}_{g}(M: \vec{f}, \vec{p})$ and $\mathcal{M}_{J}\left(T^{*} M\right.$ : $\vec{\Lambda}, \vec{x})$ for the case $k=3$. We first recall the definitions of $\mathcal{M}_{g}(M: \vec{f}, \vec{p})$ and $\mathcal{M}_{J}\left(T^{*} M\right.$ : 
$\vec{\Lambda}, \vec{x})$ for $k=3$, where

$$
\vec{f}=\left(f_{1}, f_{2}, f_{3}\right), \vec{p}=\left(p_{1}, p_{2}, p\right), \vec{\Lambda}=\left(\Lambda_{1}, \Lambda_{2}, \Lambda_{3}\right) \text { and } \vec{x}=\left(x_{1}, x_{2}, x_{3}\right) .
$$

For a given tree $T$ with 3 edges,

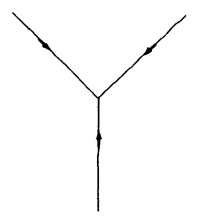

Figure 3.1

we identify (or give coordinates of) each edge with $(-\infty, 0]$. For each given metric $g$ on $M$, we consider the map

$$
I: T \rightarrow M
$$

such that the restriction $\chi_{i}=\left.I\right|_{e_{i}}$ to each edge $e$ satisfies the equation

$$
\left\{\begin{array}{l}
\frac{d \chi_{i}}{d t}=-\operatorname{grad}_{g}\left(f_{i+1}-f_{i}\right) \\
\lim _{\tau \rightarrow-\infty} \chi_{i}(\tau)=p_{i}
\end{array}\right.
$$

where $e_{i}$ is the edge between $i^{\text {th }}$ and $(i+1)^{\text {th }}$ domains with $i$ counted $\bmod 3$. By definition, $\mathcal{M}_{g}(M: \vec{f}, \vec{p})$ is the set of all such maps $I$ as above. Geometrically, one can also identify this set with

$$
\bigcap_{i=1}^{3} W_{p_{i}}^{-}\left(f_{i+1}-f_{i}\right)
$$

where $W_{p}^{-}(h)$ is the unstable manifold of the gradient flow of the function $h$ at the critical point $p \in M$.

Next, we describe $\mathcal{M}_{J}\left(T^{*} M: \vec{\Lambda}, \vec{x}\right)$. We denote by $D^{2}$ the closed unit disc and let $\left\{z_{1}, z_{2}, z_{3}\right\} \subset \partial D^{2}$ be three distinct fixed points in $\partial D^{2}$ in the cyclic order with respect to the orientation of $\partial D^{2}$ induced from the complex orientation of $D^{2} \subset \mathbb{C}$. It is convenient and essential for the later analysis to conformally identify $D^{2} \backslash\left\{z_{1}, z_{2}, z_{3}\right\}$ with a domain, denoted by $\Theta$, with 3 cylindrical ends, see Figure 3.2.

We denote the three boundary components of $\Theta$ by $\ell_{1}, \ell_{2}$ and $\ell_{3}$ denoted as in Figure 3.2. We will also denote by $\infty_{i}$ the point at infinity in $\Theta$ that corresponds to the point $z_{i}$ in $D^{2}$ under the given conformal identification.

Now for a given almost complex structure $J$ that is compatible with the canonical symplectic structure $w$ on $X=T^{*} M$, we define the set

$$
\begin{aligned}
\mathcal{M}_{J} & =\mathcal{M}_{J}(X: \vec{\Lambda}) \\
& =\left\{w: \Theta \rightarrow X \mid \bar{\partial}_{J} w=0, w\left(\ell_{i}\right) \subset \Lambda_{i} \text { and } \int_{\Theta} w^{*} \omega<\infty\right\}
\end{aligned}
$$

and for given $\vec{x}=\left(x_{1}, x_{2}, x_{3}\right)$ with $x_{i} \in \Lambda_{i} \cap \Lambda_{i+1}$,

$$
\mathcal{M}_{J}(\vec{x})=\mathcal{M}_{J}(X: \vec{\Lambda}, \vec{x})=\left\{w \in \mathcal{M}_{J}(X: \vec{\Lambda}) \mid w\left(\infty_{i}\right)=x_{i}, i=1,2,3\right\} .
$$

We will be particularly interested in the Lagrangians

$$
\Lambda_{i}^{\epsilon}:=\operatorname{Graph}\left(\epsilon d f_{i}\right), \quad i=1,2,3
$$




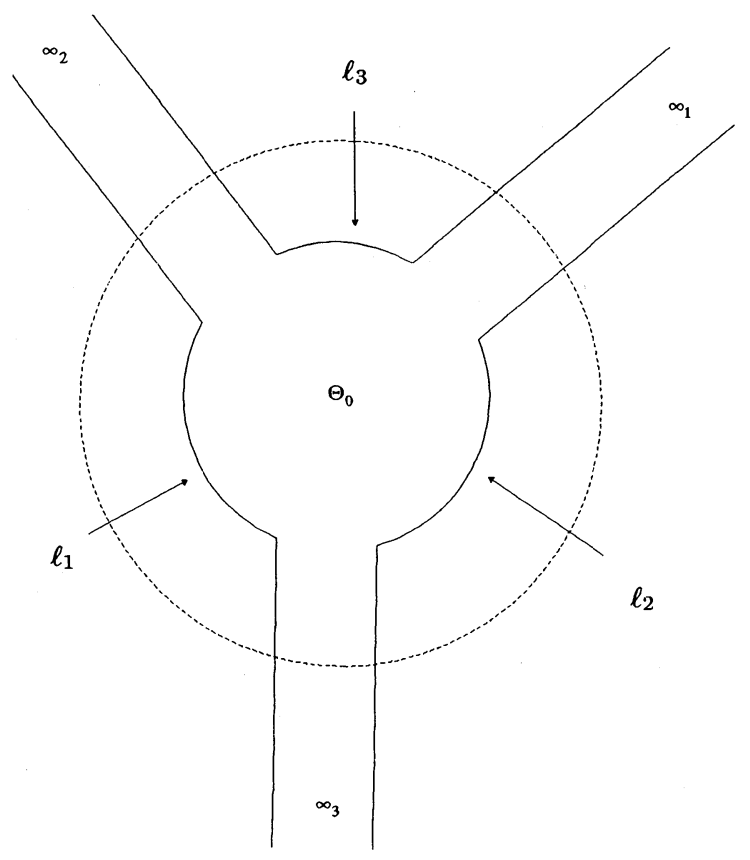

FIgURE 3.2

for a small positive parameter $\epsilon>0$. We also fix the canonical almost complex structure $J=J_{g}$ associated to the metric $g$ on $M$.

The main goal of Part I is to prove the following theorem.

TheOREM 3.1. Let $g$ be a fixed Riemannian metric on $g$ and $J=J_{g}$ be the associated canonical almost complex structure on $T^{*} M$ defined as in (1.4). Suppose that $f_{i+1}-f_{i}$ are Morse functions and that the unstable manifolds $W_{p}^{-}\left(f_{i+1}-f_{i}\right)$ of the gradient flow of $f_{i}$ 's for $i=1,2,3(\bmod 3)$ intersect transversely, i.e., we have

$$
\prod_{i=1}^{3}\left(W_{p_{i}}^{-}\left(f_{i+1}-f_{i}\right)\right) \pitchfork \Delta \text { in } M \times M \times M
$$

where $\Delta \subset M \times M \times M$ is the diagonal $\Delta=\{(q, q, q) \mid q \in M\}$. Then there exists some $\epsilon_{0}>0$ such that for any $0<\epsilon<\epsilon_{0}$ we have a diffeomorphism

$$
\Phi^{\epsilon}: \mathcal{M}_{g}(M: \vec{f}, \vec{p}) \rightarrow \mathcal{M}_{J}\left(X: \vec{\Lambda}^{\epsilon}, \vec{x}^{\epsilon}\right):=\mathcal{M}_{J}^{\epsilon}
$$

where

$$
\vec{\Lambda}^{\epsilon}=\left(\Lambda_{1}^{\epsilon}, \Lambda_{2}^{\epsilon}, \Lambda_{3}^{\epsilon}\right) \quad \vec{x}^{\epsilon}=\left(x_{1}^{\epsilon}, x_{2}^{\epsilon}, x_{3}^{\epsilon}\right) .
$$

Here we note that if $\epsilon$ is sufficiently small, there is a natural one-to-one correspondence between the sets $\operatorname{Crit}\left(f_{i+1}-f_{i}\right)$ and $\Lambda_{i+1}^{\epsilon} \cap \Lambda_{i}^{\epsilon}$. The $x_{i}^{\epsilon}$ 's above are those corresponding to $p_{i}$ 's respectively. In fact, we have

$$
x_{i}^{\epsilon}=\left(p_{i}, \epsilon d f_{i}\left(p_{i}\right)\right) .
$$

We will prove this theorem by a version of the gluing construction to produce elements in $\mathcal{M}_{J}\left(X: \vec{\Lambda}^{\epsilon}, \vec{x}^{\epsilon}\right)$ whose images are close to those in $\mathcal{M}_{g}(M: \vec{f}, \vec{p})$. There 
are two subtleties in this proof: The first one is to deal with a degeneration into one dimensional objects, which requires delicate estimates involving weighted norms in the proof. The second is more serious, in that it is not obvious at all at first sight what we should glue near the intersection point of the gradient lines to produce approximate solutions. In most of other gluing problems, it has been quite clear to guess what the appropriate approximate solutions should be.

Now we explain the analytic set-up we are going to use in the gluing construction mentioned above. We denote by $\Theta_{i}$ the $i^{\text {th }}$ cylindrical region of $\Theta$ and give the coordinates $(\tau, t)$ on $\Theta_{i} \cong(-\infty, 0) \times[0,1]$. We also denote

$$
\begin{aligned}
& \Theta_{i}(\delta)=\left\{z \in \Theta_{i} \mid-\infty<\tau<-\delta\right\}, i=1,2,3 \\
& \Theta_{0}(\delta)=\Theta \backslash \bigcup_{i=1}^{3} \overline{\Theta_{i}(\delta)}
\end{aligned}
$$

and

$$
\Theta_{i}\left(\delta_{1}, \delta_{2}\right)=\left\{z \in \Theta_{i} \mid-\delta_{2}<\tau<-\delta_{1}\right\}
$$

for $\delta$ 's positive. We choose a metric on $X=T^{*} M$, which is compatible with the symplectic structure $\omega$ so that the Lagrangians $\Lambda_{i}$ are totally geodesic near intersection points. Note that when we consider a family of Lagrangians $\Lambda_{i}^{\epsilon}$, we have to vary the metric to make the latter condition satisfied. If $w\left(\infty_{i}\right)=x_{i}, i=1,2,3$, then we can express

$$
w(\tau, t)=\exp _{x_{i}} \xi(\tau, t)
$$

for some $\xi$ that satisfies Lagrangian boundary conditions

$$
\xi(\tau, 0) \in T_{x_{i}} \Lambda_{i}, \quad \xi(\tau, 1) \in T_{x_{i}} \Lambda_{i+1} .
$$

This is because we require that $\Lambda_{i}$ 's are totally geodesic near the intersection point with respect to the metric $g$. We now define

$$
\begin{gathered}
\mathcal{F}_{\epsilon}^{1, p}=\mathcal{F}_{\epsilon}^{1, p}(X: \vec{\Lambda}, \vec{x})=\left\{w: \Theta \rightarrow X \mid w\left(\ell_{i}\right) \subset \Lambda_{i}, w=\exp _{x_{i}} \xi\right. \text { with } \\
\left.\left\|\left.\xi\right|_{\Theta_{i}(R)}\right\|_{1, p, \epsilon}<\infty \text { for some } R>0\right\}
\end{gathered}
$$

where we define the norm $\|\cdot\|_{1, p, \epsilon}$ as follows:

$$
\|\xi\|_{0, p, \epsilon}=\left(\int_{\Theta} \epsilon^{2}|\xi|^{p}\right)^{1 / p}
$$

and

$$
\|\xi\|_{1, p, \epsilon}=\left(\int_{\Theta} \epsilon^{2}|\xi|^{p}+\epsilon^{2-p}|\nabla \xi|^{p}\right)^{1 / p}
$$

Similarly for one forms $\eta \in \Omega^{1}\left(w^{*} T X\right)$, we define

$$
\begin{aligned}
\|\eta\|_{0, p, \epsilon} & =\left(\int_{\Theta} \epsilon^{2-p}|\eta|^{p}\right)^{1 / p} \\
\|\eta\|_{1, p, \epsilon} & =\left(\int_{\Theta} \epsilon^{2-p}|\eta|^{p}+\epsilon^{2-2 p}|\nabla \eta|^{p}\right)^{1 / p} .
\end{aligned}
$$

One crucial point of taking these norms is that the ordinary Sobolev norm of the rescaled $\xi, \widetilde{\xi}(u):=\xi\left(\frac{u}{\epsilon}\right)$ is the same as the weighted norm of $\xi$, i.e

$$
\|\widetilde{\xi}\|_{k, p ;[0, \epsilon]}=\|\xi\|_{k, p, \epsilon ;[0,1]} .
$$


The same applies to $\eta$. Choosing right weighted norms is the most convenient way of dealing with general singular limit problems like ours. As in [F11], one can prove that $\mathcal{F}_{\epsilon}^{1, p}$ becomes a Banach manifold modeled by $W_{\epsilon}^{1, p}\left(w^{*} T X\right)$,

$$
W_{\epsilon}^{1, p}\left(w^{*} T X\right):=\left\{\xi \in \Lambda\left(w^{*} T X\right) \mid \xi\left(\ell_{i}\right) \subset T \Lambda_{i},\|\xi\|_{1, p, \epsilon}<\infty\right\}
$$

and the map

$$
\bar{\partial}_{J}: \mathcal{F}_{\epsilon}^{1, p} \rightarrow \widetilde{\mathcal{H}}_{\epsilon}^{p}\left(w^{*} T X\right):=L_{\epsilon}^{p}\left(\Lambda_{J}^{(0,1)} T^{*} \Theta \otimes w^{*} T X\right)
$$

becomes a smooth section of the vector bundle

$$
\pi: \widetilde{\mathcal{H}}_{\epsilon}^{p} \rightarrow \mathcal{F}_{\epsilon}^{1, p}
$$

where

$$
\tilde{\mathcal{H}}_{\epsilon}^{p}=\bigcup_{w \in \mathcal{F}_{\epsilon}^{1, p}} \tilde{\mathcal{H}}_{\epsilon}^{p}\left(w^{*} T X\right)
$$

The following propositions will be the main tools to prove Theorem 3.1, which are well-known tools in the literature. Here, we adapt Theorem 3.34 and Proposition 3.35 in [MS] to our purpose.

Proposition 3.2 [Theorem 3.34, [MS]] Let $p>2$. Then for every constant $c_{0}>0$, there exist constants $\delta>0$ and $C>0$ such that the following holds. Let $w: \Theta \rightarrow X$ be a map in $\mathcal{F}_{\epsilon}^{1, p}$ and

$$
Q_{w}: \tilde{\mathcal{H}}_{\epsilon}^{p} \rightarrow T_{w} \mathcal{F}_{\epsilon}^{1, p}=W_{\epsilon}^{1, p}\left(w^{*} T X\right)
$$

be a right inverse of

$$
D_{w}:=D \bar{\partial}_{J}(w): T_{w} \mathcal{F}_{\epsilon}^{1, p} \rightarrow \widetilde{\mathcal{H}}_{\epsilon}^{p}
$$

such that $D_{w} \circ Q_{w}=$ id and

$$
\left\|Q_{w}\right\| \leq c_{0},\|D w\|_{L_{\epsilon}^{p}} \leq c_{0},\left\|\bar{\partial}_{J}(w)\right\|_{L_{\epsilon}^{p}} \leq \delta .
$$

Then for every $\xi \in \operatorname{Ker} D_{w}$ with $\|\xi\|_{1, p, \epsilon} \leq \delta$, there exists a section $\hat{\xi}=Q_{w} \eta \in$ $W_{\epsilon}^{1, p}\left(w^{*} T X\right)$ such that

$$
\bar{\partial}_{J}\left(\exp _{w}\left(\xi+Q_{w} \eta\right)\right)=0,\left\|Q_{w} \eta\right\|_{1, p, \epsilon} \leq C\left\|\bar{\partial}_{J}\left(\exp _{w} \xi\right)\right\|_{0, p, \epsilon} .
$$

Proposition 3.3 [Proposition 3.35 [MS]] Let $p>2$. Then for every constant $c_{0}>0$ there exists a constant $\delta>0$ such that the following holds. Let $w: \Theta \rightarrow X$ and $Q_{w}: \widetilde{\mathcal{H}}_{\epsilon}^{p} \rightarrow T_{w} \mathcal{F}_{\epsilon}^{1, p}$ such that $w \in \mathcal{F}_{\epsilon}^{1, p}, D_{w} \circ Q_{w}=i d$ and

$$
\left\|Q_{w}\right\| \leq c_{0},\|D w\|_{0, p, \epsilon} \leq c_{0} .
$$

If $w_{0}=\exp _{w}\left(\xi_{0}\right)$ and $w_{1}=\exp _{w}\left(\xi_{1}\right)$ are J-holomorphic maps such that $\xi_{0}, \xi_{1} \in$ $W_{\epsilon}^{1, p}\left(w^{*} T X\right)$ satisfy

$$
\left\|\xi_{0}\right\|_{1, p, \epsilon} \leq \delta,\left\|\xi_{1}\right\|_{1, p, \epsilon} \leq c_{0}
$$

and

$$
\left\|\xi_{1}-\xi_{0}\right\|_{\infty} \leq \delta, \quad \xi_{1}-\xi_{0} \in \operatorname{Im} Q_{w}
$$

then

$$
w_{0}=w_{1} .
$$

The following lemma is also useful in later computations, which is a standard fact in symplectic geometry. We omit the proof. 
Lemma 3.4 Let $g$ and $h$ be a two smooth functions on $M$ and $G$ and $H$ be their lifts to $T^{*} M$ i.e

$$
G(x)=g(\pi x) \quad(\text { resp. } H(x)=h(\pi x)) .
$$

Then the Poisson bracket $\{G, H\}$ satisfies

$$
\{G, H\}=0
$$

and so their Hamiltonian flows $\phi_{t}^{g}$ and $\phi_{t}^{h}$ commute. In particular, we have

$$
\left(\phi_{t}^{h}\right)^{*} X_{G}=X_{G}
$$

4. Construction of approximate solutions. We divide $\Theta$ into 4 main regions and 3 intermediate regions which vary depending on $\epsilon>0$. We will choose a positive constant $\alpha$ such that

$$
0<\alpha<1
$$

in the rest of Part I. In Part II, we have to vary $\alpha$ depending on how fast conformal structures of the domain $\Theta$ with $k>3$ degenrate. With this constant $\alpha$, we consider for $i=1,2,3$,

$$
\Theta_{i}\left(\frac{2}{\epsilon^{\alpha}}\right)=\left\{z \in \Theta_{i} \mid-\infty<\tau<-\frac{2}{\epsilon^{\alpha}}\right\}
$$

and

$$
\Theta_{0}\left(\frac{1}{\epsilon^{\alpha}}\right)=\Theta \backslash \cup_{i=1}^{3} \overline{\Theta_{i}\left(\frac{1}{\epsilon^{\alpha}}\right)} .
$$

We will describe the possible approximate solutions $w_{\epsilon}$ on each of these regions separately and then interpolate them on the remaining regions of $\Theta$.

We start with the regions $\Theta_{i}\left(\frac{2}{\epsilon^{\alpha}}\right), i=1,2,3$. For each given Morse function $h$ on $M \subset T^{*} M$, we define the Hamiltonian $H: X \rightarrow \mathbb{R}$ by

$$
H(x):=h(\pi x)
$$

where $\pi: T^{*} M \rightarrow M$ is the canonical projection. We denote by $\phi_{t}^{h}$ the Hamiltonian flow of $H$. In the regions $\Theta_{i}\left(\frac{2}{\epsilon^{\alpha}}\right)$, we just define

$$
w_{i}^{\epsilon}(z)=w_{i}^{\epsilon}(\tau+i t)=\phi_{\epsilon t}^{f_{i+1}} \phi_{\epsilon(1-t)}^{f_{i}}\left(\chi_{i}(\epsilon \tau)\right)
$$

for each given $I \in \mathcal{M}(M: \vec{f}, \vec{p})$, where we recall

$$
\chi_{i}:=\left.I\right|_{e_{i}} .
$$

One can easily check that $w_{\epsilon}$ satisfies the required boundary condition

$$
w_{i}^{\epsilon}(\tau, 0) \in \Lambda_{i}^{\epsilon}, w_{i}^{\epsilon}(\tau, 1) \in \Lambda_{i+1}^{\epsilon}, i=1,2,3 .
$$

To describe the part of $w^{\epsilon}$ on $\Theta_{0}\left(\frac{1}{\epsilon^{\alpha}}\right)$, we first re-scale a neighborhood of each given intersection point $x \in \cap_{i=1}^{3} W_{p_{i}}^{-}\left(f_{i+1}-f_{i}\right) \subset M \subset T^{*} M$ in $X=T^{*} M$. We consider the exponential map

$$
\exp _{x}: T_{x} X \rightarrow X
$$

and denote

$$
\widetilde{\Lambda}_{i}^{\epsilon}:=\frac{1}{\epsilon} \exp _{x}^{-1}\left(\Lambda_{i}^{\epsilon}\right) \cap B_{\epsilon^{1-\alpha}}(0) \subset T_{x} X .
$$

One can easily see that as $\epsilon \rightarrow 0$,

$$
\widetilde{\Lambda}_{i}^{\epsilon} \rightarrow \widetilde{\Lambda}_{i}=\left\{(q, p) \in T_{x} X \cong \mathbb{R}^{2 n} \mid p=\nabla f_{i}(x)\right\}
$$


uniformly on compact sets. We will first construct a holomorphic map

$$
\widetilde{w}_{0}: \Theta \rightarrow \mathbb{C}^{n} \cong \mathbb{R}^{2 n} \cong T_{x} X
$$

with boundary conditions

$$
\widetilde{w}_{0}\left(\ell_{i}\right) \subset \widetilde{\Lambda}_{i}, \quad i=1,2,3,
$$

and with appropriate asymptotic conditions at each end, which we now describe.

Since we are going to glue the part $w_{0}^{\epsilon}$ on $\Theta_{0}\left(\frac{1}{\epsilon^{\alpha}}\right)$ to $w_{i}^{\epsilon}$ 's defined in (4.1), the asymptotic conditions of $w_{0}^{\epsilon}$ should match those (rescaled by $\epsilon$ ) obtained from

$$
w_{i}^{\epsilon}\left(\frac{1}{\epsilon^{\alpha}}+i t\right)=\phi_{\epsilon t}^{f_{i+1}} \phi_{\epsilon(1-t)}^{f_{i}}\left(\chi_{i}\left(\epsilon^{1-\alpha}\right)\right) .
$$

We identify $T_{x} X$ with $\mathbb{C}^{n}$ so that $T_{x} M \subset T_{x} X$ becomes the real plane $\mathbb{R}^{n}$ and $J$. $T_{x} M \subset T_{x} X$ becomes the imaginary plane $i \cdot \mathbb{R}^{n} \subset \mathbb{C}^{n}$. We denote the real and imaginary parts of $v \in \mathbb{C}^{n}$ by $\operatorname{Re} v$ and $\operatorname{Im} v$ respectively. With this notation, it is now easy to check that we have

$$
\lim _{\epsilon \rightarrow 0} \frac{1}{\epsilon} \operatorname{Im}\left\{\exp _{x}^{-1}\left(w_{i}^{\epsilon}\left(\frac{1}{\epsilon^{\alpha}}+i t\right)\right)\right\}=t\left(\nabla f_{i+1}(x)-\nabla f_{i}(x)\right)+\nabla f_{i}(x) .
$$

Therefore, a natural candidate for the needed asymptotic condition will be

$$
\left.\lim _{\tau \rightarrow-\infty} \operatorname{Im} \widetilde{w}_{0}\right|_{\Theta_{i}}(\tau, t)=t\left(\nabla f_{i+1}(x)-\nabla f_{i}(x)\right)+\nabla f_{i}(x)
$$

uniformly over $t \in[0,1]$. We now prove that this is precisely the natural asymptotic condition we should impose on $\widetilde{w}_{0}$.

Proposition 4.1. The solution set of $\tilde{w}_{i}: \Theta \rightarrow \mathbb{C}^{n}$ satisfying

$$
\left\{\begin{array}{r}
\bar{\partial} \widetilde{w}_{0}=0, \quad \widetilde{w}_{0}\left(\ell_{i}\right) \subset \widetilde{\Lambda}_{i} \\
\left.\lim _{\tau \rightarrow-\infty} \operatorname{Im} \bar{w}_{0}\right|_{\Theta_{i}}(\tau, t)=t\left(\nabla f_{i+1}(x)-\nabla f_{i}(x)\right)+\nabla f_{i}(x), \\
\text { for } 1=1,2,3
\end{array}\right.
$$

is unique (if it exists) up to addition by real constant vectors.

Proof. Suppose that $\widetilde{w}_{0}$ and $\widetilde{w}_{0}^{\prime}$ be two such solutions. We consider the difference map

$$
\xi=\widetilde{w}_{0}-\widetilde{w}_{0}^{\prime}: \Theta \rightarrow \mathbb{C}^{n}
$$

Since $\widetilde{\Lambda}_{i}$ are affine spaces given by

$$
\widetilde{\Lambda}_{i}=\left\{(q, p) \in \mathbb{R}^{2 n} \cong \mathbb{C}^{n} \mid p=\nabla f_{i}(x)\right\}
$$

$\xi$ satisfies

$$
\xi\left(\ell_{i}\right) \subset \mathbb{R}^{n}, \quad i=1,2,3 .
$$

Furthermore, it also satisfies the asymptotic condition

$$
\left.\lim _{\tau \rightarrow \infty} \operatorname{Im} \xi\right|_{\Theta_{i}}=0 \quad \text { uniformly. }
$$

Therefore $\xi: \Theta \rightarrow \mathbb{C}^{n}$ is a holomorphic map such that

$$
\left.\operatorname{Im} \xi\right|_{\partial \Theta} \equiv 0
$$

and $|\operatorname{Im} \xi|$ is bounded on $\Theta$. Applying the maximum principle to the harmonic map $\operatorname{Im} \xi$ on $\Theta$ into $\mathbb{C}^{n}$, we conclude

$$
\operatorname{Im} \xi \equiv 0 \quad \text { on } \Theta
$$


which will in turn imply that

$\xi \equiv$ a real constant vector.

This finishes the proof.

Now, we will remove non-uniqueness in this proposition by imposing the following balancing condition (4.7). This will be important in finding a good approximate solution which enables us to obtain necessary error estimates. Since $\widetilde{w}_{0}$ is holomorphic and satisfies

$$
\left.\lim _{\tau \rightarrow-\infty} \operatorname{Im} \tilde{w}_{0}\right|_{\Theta_{i}}(\tau, t)=t\left(\nabla f_{i+1}(x)-\nabla f_{i}(x)\right)+\nabla f_{i}(x)
$$

which is a "linear" function on $t, \widetilde{w}_{0}$ must satisfy

$$
\left|\widetilde{w}_{0}(\tau, t)-v_{j}+i \nabla f_{j}(x)+(\tau+i t)\left(\nabla f_{i+1}(x)-\nabla f_{i}(x)\right)\right| \rightarrow 0
$$

uniformly as $\tau \rightarrow \infty$ for some vectors $v_{j} \in \mathbb{R}^{n}, j=1,2,3$. We note that the direction vectors $\left(\nabla f_{i+1}(x)-\nabla f_{i}(x)\right)$ satisfy

$$
\left(\nabla f_{2}(x)-\nabla f_{1}(x)\right)+\left(\nabla f_{3}(x)-\nabla f_{2}(x)\right)+\left(\nabla f_{1}(x)-\nabla f_{3}(x)\right)=0 .
$$

We remove the ambiguity in Proposition 4.1 by imposing the condition

$$
\left.\lim _{\tau \rightarrow-\infty} \sum_{j=1}^{3} \operatorname{Re} \widetilde{w}_{0}\right|_{\Theta_{i}}(\tau, t)=0 .
$$

This can be always achieved, due to (4.6), by choosing appropriate real vectors $v_{j}$ 's in (4.5).

It remains to prove existence of a solution to (4.4). For notational convenience, we denote

$$
u_{j}=\nabla f_{j+1}(x)-\nabla f_{j}(x), \quad j=1,2,3
$$

and then the span of $u_{j}$ 's satisfy

$$
\operatorname{dim}_{\mathbb{R}} \operatorname{span}_{\mathbb{R}}\left\{u_{1}, u_{2}, u_{3}\right\}=2
$$

because $u_{1}+u_{2}+u_{3}=0$ from (4.6). By the uniqueness theorem, it will be enough (if possible) to construct a solution of (4.4) such that

$$
\text { Image } \widetilde{w}_{0} \subset \operatorname{Span}_{\mathbb{C}}\left\{u_{1}, u_{2}, u_{3}\right\}+i \nabla f_{1}(x) .
$$

We denote the affine space of complex dimension 2

$$
W:=\operatorname{Span}_{\mathbb{C}}\left\{u_{1}, u_{2}, u_{3}\right\}+i \nabla f_{1}(x) \subset \mathbb{C}^{n} .
$$

We will assume without loss of any generality that $\nabla f_{1}(x)=0$ and so $W$ becomes a subspace. In this way, we have reduced the existence problem to one in $W \cong \mathbb{C}^{2}$. If we denote

$$
V=\operatorname{Span}_{\mathbb{R}}\left\{u_{1}, u_{2}, u_{3}\right\} \subset \mathbb{R}^{n}
$$

we have

$$
W=V_{\mathbb{C}}+i \nabla f_{1}(x)
$$

where $V_{\mathbb{C}}$ is the complexification of $V$. We now consider two complex projection

$$
\pi_{1}, \pi_{2}: W \rightarrow W
$$


such that Image $\pi_{i}$ are one dimensional and

$$
\begin{array}{ll}
\pi_{1}=\text { projection along } & u_{1}=\nabla f_{2}(x)-\nabla f_{1}(x) \\
\pi_{2}=\text { projection along } & u_{2}=\nabla f_{3}(x)-\nabla f_{2}(x) .
\end{array}
$$

By identifying the images of $\pi_{i}$ 's with $\mathbb{C}$, we have coordinates which we denote by

$$
\left(\pi_{1}, \pi_{2}\right) \in \mathbb{C}^{2} \text {. }
$$

To determine $\widetilde{w}_{0}: \Theta \rightarrow W \subset \mathbb{C}^{n}$, it will be enough to determine its coordinate functions $\pi_{i} \circ \widetilde{w}_{0}: \Theta \rightarrow \mathbb{C}$. Denote

$$
\tilde{V}_{j}=V+i \cdot \nabla f_{j}(x) \quad j=1,2,3 .
$$

Then it follows

$$
\begin{aligned}
& \pi_{1}\left(\widetilde{V}_{1}\right)=\pi_{1}\left(\widetilde{V}_{2}\right) \subset \pi_{1}(W) \\
& \pi_{2}\left(\widetilde{V}_{2}\right)=\pi_{2}\left(\widetilde{V}_{2}\right) \subset \pi_{2}(W) .
\end{aligned}
$$

We will now seek holomorphic functions

$$
a_{k}: \Theta \rightarrow \mathbb{C}, \quad k=1,2,
$$

such that

$$
\begin{cases}a_{1}\left(\ell_{1}\right), a_{1}\left(\ell_{2}\right) & \subset \pi_{1}\left(\tilde{V}_{1}\right)=\pi_{1}\left(\tilde{V}_{2}\right) \\ a_{1}\left(\ell_{3}\right) & \subset \pi_{1}\left(\tilde{V}_{2}\right)\end{cases}
$$

and

$$
\begin{cases}a_{2}\left(\ell_{1}\right) & \subset \pi_{2}\left(\tilde{V}_{1}\right) \\ a_{2}\left(\ell_{2}\right), a_{2}\left(\ell_{3}\right) & \subset \pi_{2}\left(\tilde{V}_{2}\right) .\end{cases}
$$

Then we will choose $\widetilde{w}_{0}: \Theta \rightarrow W \subset \mathbb{C}^{n}$ such that

$$
\widetilde{w}_{0}(z)=\left(a_{1}(z), a_{2}(z)\right)
$$

in coordinates $\left(\pi_{1}, \pi_{2}\right)$ of $W$. By conformal identification of $\Theta$ with $D^{2} \backslash\left\{z_{1}, z_{2}, z_{3}\right\}$, the above description of finding $a_{1}$ is equivalent to finding holomorphic map

$$
a_{1}: D^{2} \backslash\left\{z_{2}, z_{3}\right\} \rightarrow \mathbb{C}
$$

with

$$
\begin{cases}a_{1}\left(\ell_{1} \cup\left\{z_{1}\right\} \cup \ell_{2}\right) & \subset \pi_{1}\left(\tilde{V}_{1}\right) \\ a_{1}\left(\ell_{3}\right) & \subset \pi_{1}\left(\tilde{V}_{3}\right)\end{cases}
$$

and

$$
a_{1}\left(z_{2}\right)=-\infty, \quad a_{1}\left(z_{3}\right)=\infty .
$$

Existence of such functions immediately follows from the Riemann mapping theorem. In fact, there exists one dimensional family of such functions. Similarly, we find a holomorphic function

$$
a_{2}: \Theta \rightarrow \mathbb{C}
$$

such that

$$
\left\{\begin{array}{l}
a_{2}\left(\ell_{1}\right) \subset \pi_{2}\left(\tilde{V}_{1}\right) \\
a_{2}\left(\ell_{2} \cup \ell_{3} \cup\left\{z_{2}\right\}\right) \subset \pi_{2}\left(\tilde{V}_{2}\right)
\end{array}\right.
$$


and

$$
a_{2}\left(z_{3}\right)=-\infty, \quad a_{2}\left(z_{1}\right)=\infty .
$$

Finally, we need to check that the map $\widetilde{w}_{0}: \Theta \rightarrow \mathbb{C}$ defined by

$$
\widetilde{w}_{0}(z)=\left(a_{1}(z), a_{2}(z)\right)
$$

in coordinates $\left(\pi_{1}, \pi_{2}\right)$ of $W$ indeed satisfy all the requirements in Proposition 4.1, especially the asymptotic conditions. To check the asymptotic conditions, we recall that since $\Theta$ has cylindrical ends with the same width, it follows from the properties of the Riemann map that both $a_{1}$ and $a_{2}$ are asymptotically linear at each end. More precisely, the functions $a_{1}$ must satisfy

$$
\begin{aligned}
& \left.a_{1}\right|_{\Theta_{2}}(\tau, t) \rightarrow b(\tau+i t) \\
& \left.a_{1}\right|_{\Theta_{2}}(\tau, t) \rightarrow-b(\tau+i t) \quad \text { as }|\tau| \rightarrow \infty
\end{aligned}
$$

for a constant $b \in \mathbb{C}$. Similar conditions must hold for $a_{2}$.

Now, we consider the asymptotic conditions of $\widetilde{w}_{0}$ at each point of $z_{1}, z_{2}$ and $z_{3}$. First at $z_{1} \in \partial D^{2}$, we have, from (4.8.1), (4.9.2) and the asymptotic linearity of $a_{2}$,

$$
\begin{aligned}
& a_{1}\left(z_{1}\right) \in \pi_{1}\left(\widetilde{V}_{1}\right) \\
& a_{2}(\tau+i t) \sim b(\tau+i t) \quad \text { as }|\tau| \rightarrow \infty .
\end{aligned}
$$

We interpret these conditions for $\widetilde{w}_{0}$ in the standard coordinates on $\Theta_{1}$. It is easy to check that (4.11.1) implies that the image of $\widetilde{w}_{0}$ is asymptotically tangent to $\operatorname{span}\left\{i u_{1}\right\}$ and (4.11.2) implies that $w_{0}$ is asymptotically linear which are precisely the conditions for $\widetilde{w}_{0}$ to satisfy on $\Theta_{1}$. Similar consideration applies to $z_{2}$ and so on $\Theta_{2}$. It remains to prove the asymptotic condition on $\Theta_{3}$. At $z_{3}$, we have

$$
a_{1}\left(\ell_{3}\right) \subset \pi_{1}\left(\widetilde{V}_{3}\right), \quad a_{1}\left(\ell_{1}\right) \subset \pi_{1}\left(\tilde{V}_{1}\right)
$$

and

$$
a_{2}\left(\ell_{3}\right) \subset \pi_{2}\left(\widetilde{V}_{2}\right), \quad a_{2}\left(\ell_{1}\right) \subset \pi_{2}\left(\widetilde{V}_{1}\right) .
$$

Moreover both $a_{1}$ and $a_{2}$ are asymptotically linear at $z_{3}$. It now follows from these that $\widetilde{w}_{0}$ also satisfies the required asymptotic condition on $\Theta_{3}$. This finishes the proof of the existence of solutions satisfying the equation in Proposition 4.1.

Remark 4.2. Originally, we found the solution $\widetilde{w}_{0}$ by a different method, which first solves the minimization problem of the harmonic energy

$$
\int_{\Theta_{i}(R)}|D w|^{2}
$$

for large fixed $R>0$ with appropriate boundary conditions and then proves the minimizer must be holomorphic. Then $\widetilde{w}_{0}$ can be obtained as the limit as $R \rightarrow \infty$. This method is possible because we require that $\widetilde{w}_{0}$ satisfy the totally geodesic Lagrangian boundary condition given by $\widetilde{\Lambda}_{i}$ in $\mathbb{C}^{n}$ (See some remnants of this method in the proof of Lemma 16.3). Only after we proved the uniqueness result Proposition 4.1, we have been able to find the above elementary method.

Now, we use $\widetilde{w}_{0}: \Theta \rightarrow \mathbb{C}^{n}$ to construct the portion on $\Theta_{0}\left(\frac{1}{\epsilon^{\alpha}}\right)$ of our approximate solution

$$
w^{\epsilon}: \Theta \rightarrow X
$$


It would be very natural to define

$$
w_{0}^{\epsilon}: \Theta_{0}\left(\frac{1}{\epsilon^{\alpha}}\right) \rightarrow X
$$

by

$$
w_{0}^{\epsilon}(z)=\exp _{x} \epsilon \widetilde{w}_{0}(z) .
$$

Unfortunately, this does not quite satisfy the boundary conditions

$$
\left.w_{0}^{\epsilon}\right|_{\ell_{i}} \subset \Lambda_{i}^{\epsilon}
$$

For the moment ignore this fact and proceed defining $w^{\epsilon}$. Finally, we interpolate $w_{i}^{\epsilon}$ with $w_{0}^{\epsilon}$ for each $i=1,2,3$ on the region $\Theta_{i}\left(\frac{1}{\epsilon^{\alpha}}, \frac{2}{\epsilon^{\alpha}}\right)$. We choose a cut-off function $\beta:(-\infty, 0] \rightarrow \mathbb{R}$ such that

$$
\begin{cases}\beta=0 & \text { for }-1 \leq \tau \leq 0 \\ \beta=1 & \text { for } \tau \leq-2 \\ -2 \leq & \beta^{\prime}(\tau) \leq 0\end{cases}
$$

We denote

$$
\widetilde{w}_{i}^{\epsilon}:=\frac{1}{\epsilon} \exp _{x}^{-1} w_{i}^{\epsilon}, \quad i=1,2,3,
$$

and complete the definition of $w^{\epsilon}=w^{\epsilon, I}$ by

$$
w^{\epsilon}(z)=w^{\epsilon, I}(z)= \begin{cases}\phi_{\epsilon t}^{f_{i+1}} \circ \phi_{\epsilon(1-t)}^{f_{i}}\left(\chi_{i}(\epsilon \tau)\right) & \text { for } z \in \Theta_{i}\left(\frac{2}{\epsilon^{\alpha}}\right) \\ \exp _{x} \epsilon \widetilde{w}_{0}(z) & \text { for } z \in \Theta_{0}\left(\frac{1}{\epsilon^{\alpha}}\right) \\ \exp _{x} \epsilon\left(\widetilde{w}_{0}(z)+\beta\left(\epsilon^{\alpha} \tau\right)\left(\widetilde{w}_{i}^{\epsilon}(z)-\widetilde{w}_{0}(z)\right)\right. & \\ & \text { for } z \in \Theta_{i}\left(\frac{1}{\epsilon^{\alpha}}, \frac{2}{\epsilon^{\alpha}}\right) .\end{cases}
$$

It remains to justify the fact that this is a good approximate solution although it does not quite satisfy the boundary conditions on the regions

$$
\Theta_{i}\left(\frac{1}{\epsilon^{\alpha}}\right)
$$

First we note that since $\widetilde{w}_{0}$ is asymptotically linear and so $\left|\epsilon \widetilde{w}_{0}(z)\right| \sim \epsilon^{1-\alpha}$ on $\theta_{0}\left(\frac{1}{\epsilon^{\alpha}}\right)$, $\exp _{x} \epsilon \widetilde{w}_{0}(z) \rightarrow x$ as $\epsilon \rightarrow 0$ uniformly over $\Theta_{0}\left(\frac{1}{\epsilon^{\alpha}}\right)$ for all $x \in \cap_{j=1}^{3} W_{p_{j}}^{-}\left(f_{j+1}-f_{j}\right)$. Hence, one can correct $w^{\epsilon}$ on the image by a $C^{1}$-small perturbations so that it satisfies the correct boundary condition. Because of this, we will pretend that $w^{\epsilon}$ defined in (4.12) satisfies the correct boundary conditions.

Remark 4.3. It is important to note that because of (4.6), the images of $\epsilon \widetilde{w}_{0}(z)$ converges to the three lines intersecting at the origin in the Hausdorff sense as $\epsilon \rightarrow 0$, which are in the directions of $\operatorname{grad}\left(f_{i+1}-f_{i}\right)(x)$ for $i=1,2,3$. This point will be important in Section 6 and 7.

5. Error estimates. We start with the regions $\Theta_{i}\left(\frac{2}{\epsilon^{\alpha}}\right)$ for $i=1,2,3$. In terms of the coordinates $(\tau, t)$ on $\Theta_{i}$, we have

$$
\left|\bar{\partial}_{J} w^{\epsilon}\right|=\frac{1}{2}\left|\frac{\partial w^{\epsilon}}{\partial \tau}+J \frac{\partial w^{\epsilon}}{\partial t}\right| \quad \text { on } \Theta_{i}
$$

where the left hand side is the norm taken in $\Lambda^{(0,1)} T^{*} \Theta \otimes\left(w^{\epsilon}\right)^{*} T X$ and the right hand side is the one taken in $\left(w^{\epsilon}\right)^{*} T X$. Therefore, we will compute the right hand 
side instead of $\left|\bar{\partial}_{J} w^{\epsilon}\right|$. For notational convenience, we denote

$$
w_{i}(\tau, t):=\left.w^{\epsilon}\right|_{\Theta_{i}}(\tau, t)=\phi_{\epsilon t}^{f_{i+1}} \circ \phi_{\epsilon(1-t)}^{f_{i}}\left(\chi_{i}(\epsilon \tau)\right) \text { on } \Theta_{i} .
$$

We compute

$$
\begin{aligned}
\frac{\partial w_{i}}{\partial \tau} & =\epsilon T \phi_{\epsilon t}^{f_{i+1}} \circ T \phi_{\epsilon(1-t)}^{f_{i}}\left(\chi_{i}^{\prime}(\epsilon \tau)\right) \\
& =\epsilon T \phi_{\epsilon t}^{f_{i+1}} \circ T \phi_{\epsilon(1-t)}^{f_{i}}\left(-\operatorname{grad}\left(f_{i+1}-f_{i}\right)\left(\chi_{i}(\epsilon \tau)\right)\right.
\end{aligned}
$$

where the second equality comes from (3.1). And

$$
\begin{aligned}
\frac{\partial w_{i}}{\partial t} & =\epsilon X_{F_{i+1}}\left(w_{i}\right)-\epsilon T \phi_{\epsilon t}^{f_{i+1}} X_{F_{i}}\left(\left(\phi_{\epsilon t}^{f_{i+1}}\right)^{-1}\left(w_{i}\right)\right) \\
& =\epsilon X_{F_{i+1}}\left(w_{i}\right)-\epsilon X_{F_{i}}\left(w_{i}\right)=\epsilon X_{\left(F_{i+1}-F_{i}\right)}\left(w_{i}\right) \\
& =\epsilon T \phi_{\epsilon t}^{f_{i+1}} \circ T \phi_{\epsilon(1-t)}^{f_{i}}\left(X_{\left(F_{i+1}-F_{i}\right)}\left(\chi_{i}(\epsilon \tau)\right)\right)
\end{aligned}
$$

where we used the identities

$$
\begin{gathered}
\left(\phi_{\epsilon t}^{f_{i+1}}\right)^{*} X_{F_{i+1}}=X_{F_{i+1}} \\
X_{H}-X_{G}=X_{(H-G)} .
\end{gathered}
$$

Therefore we have

$$
\begin{aligned}
& \frac{\partial w_{i}}{\partial \tau}+J \frac{\partial w_{i}}{\partial t}=-\epsilon T \phi_{\epsilon t}^{f_{i+1}} \circ T \phi_{\epsilon(1-t)}^{f_{i}}\left(\operatorname{grad}\left(f_{i+1}-f_{i}\right)\left(\chi_{i}(\epsilon \tau)\right)\right) \\
& +\epsilon J T \phi_{\epsilon t}^{f_{i+1}} \circ T \phi_{\epsilon(1-t)}^{f_{i}} X_{\left(F_{i+1}-F_{i}\right)}\left(\chi_{i}(\epsilon \tau)\right) \\
& =-\epsilon T \phi_{\epsilon t}^{f_{i+1}} \circ T \phi_{\epsilon(1-t)}^{f_{i}} J X_{\left(F_{i+1}-f_{i}\right)}\left(\chi_{i}(\epsilon \tau)\right) \\
& +\epsilon J T \phi_{\epsilon t}^{f_{i+1}} \circ T \phi_{\epsilon(1-t)}^{f_{i}} X_{\left(F_{i+1}-F_{i}\right)}\left(\chi_{i}(\epsilon \tau)\right) \\
& =-\epsilon T \phi_{\epsilon t}^{f_{i+1}} \circ T \phi_{\epsilon(1-t)}^{f_{i}}\left\{J X_{\left(F_{i+1}-F_{i}\right)}-\right. \\
& \left.-\left(\phi_{\epsilon t}^{f_{i+1}} \circ \phi_{\epsilon(1-t)}^{f_{i}}\right)^{*} J \cdot X_{\left(F_{i+1}-F_{i}\right)}\right\}\left(\chi_{i}(\epsilon \tau)\right) \text {. }
\end{aligned}
$$

Here we used Lemma 3.4 and the identity

$$
J X_{\left(F_{i+1}-F_{i}\right)}=\operatorname{grad}\left(f_{i+1}-f_{i}\right)
$$

on $M \subset T^{*} M$. Since we have

$$
\left|\phi_{\epsilon t}^{f_{i+1}}-\mathrm{id}\right|_{C^{1}},\left|\phi_{\epsilon(1-t)}^{f_{i}}-\mathrm{id}\right|_{C^{1}} \leq C \epsilon
$$

where $C$ is the constant depending only on $\vec{f}=\left(f_{1}, f_{2}, f_{3}\right)$, we have

$$
\begin{aligned}
& \left|\frac{\partial w_{i}}{\partial \tau}+J \frac{\partial w_{i}}{\partial t}\right|(\tau, t) \\
& \quad \leq C \epsilon\left|J X_{\left(F_{i+1}-F_{i}\right)}-\left(\phi_{\epsilon t}^{f_{i+1}} \circ \phi_{\epsilon(1-t)}^{f_{i}}\right)^{*} J \cdot X_{\left(F_{i+1}-F_{i}\right)}\right|\left(\chi_{i}(\epsilon \tau)\right) .
\end{aligned}
$$

For the simplicity of exposition, we denote

$$
\begin{aligned}
Y_{i, \epsilon} & :=J X_{\left(F_{i+1}-F_{i}\right)}-\left(\phi_{\epsilon t}^{f_{i+1}} \circ \phi_{\epsilon(1-t)}^{f_{i}}\right)^{*} J \cdot X_{\left(F_{i+1}-F_{i}\right)} \\
& =\left(J-\left(\phi_{\epsilon t}^{f_{i+1}} \circ \phi_{\epsilon(1-t)}^{f_{i}}\right)^{*} J\right) \cdot X_{\left(F_{i+1}-F_{i}\right)}
\end{aligned}
$$


and then, we have

$$
\begin{aligned}
& \left\|\bar{\partial}_{J} w^{\epsilon}\right\|_{0, p, \epsilon, \Theta_{i}\left(\frac{2}{\epsilon^{\alpha}}\right)}^{p}=\int_{\Theta_{i}\left(\frac{2}{\epsilon^{\alpha}}\right)} \epsilon^{2-p}\left|\bar{\partial}_{J} w_{\epsilon}\right|^{p} \\
& \leq C^{p} \epsilon^{2} \int_{\Theta_{i}\left(\frac{2}{\epsilon^{\alpha}}\right)}\left|Y_{i, \epsilon}\right|^{p}\left(\chi_{i}(\epsilon \tau)\right) \\
& \quad=C^{p} \epsilon^{2} \int_{0}^{1} \int_{-\infty}^{-\frac{2}{\epsilon^{\alpha}}}\left|Y_{i, \epsilon}\right|^{p}\left(\chi_{i}(\epsilon \tau)\right) d \tau d t \\
& \quad=C^{p} \epsilon \int_{0}^{1} \int_{-\infty}^{-2 \epsilon^{1-\alpha}}\left|Y_{i, \epsilon}\right|^{p}\left(\chi_{i}(\sigma)\right) d \sigma d t, \sigma=\epsilon \tau \\
& \leq C^{p} \epsilon \int_{0}^{1} \int_{-\infty}^{0}\left|Y_{i, \epsilon}\right|^{p}\left(\chi_{i}(\sigma)\right) d \sigma d t \\
& \leq C^{p} \epsilon \sup _{x \in M}\left|J-\left(\phi_{\epsilon t}^{f_{i+1}} \circ \phi_{\epsilon(1-t)}^{f_{i}}\right)^{*} J\right|^{p}(x) \int_{-\infty}^{0}\left|X_{\left(F_{i+1}-F_{i}\right)}\right|^{p}\left(\chi_{i}(\sigma)\right) d \sigma .
\end{aligned}
$$

Here it is easy to see that

$$
\sup _{x \in M}\left|J-\left(\phi_{\epsilon t}^{f_{i+1}} \circ \phi_{\epsilon(1-t)}^{f_{i}}\right)^{*} J\right| \leq C \epsilon
$$

and so we have

$$
\left\|\bar{\partial}_{J} w^{\epsilon}\right\|_{0, p, \epsilon, \Theta_{i}\left(\frac{2}{\epsilon^{\alpha}}\right)}^{p} \leq \widetilde{C}^{p} \epsilon^{1+p} \int_{-\infty}^{0}\left|X_{\left(F_{i+1}-F_{i}\right)}\right|^{p}\left(\chi_{i}(\sigma)\right) d \sigma .
$$

Since $J X_{\left(F_{i+1}-F_{i}\right)}=\operatorname{grad}\left(f_{i+1}-f_{i}\right)$ on $M \subset T^{*} M$ and the gradient trajectory $\chi_{i}=$ $\left.I\right|_{e_{i}}$ converges exponentially to $p_{i}$ as $\sigma \rightarrow-\infty$, we have

$$
\left|X_{\left(F_{i+1}-F_{i}\right)}\right|\left(\chi_{i}(\sigma)\right)=O\left(e^{-C \sigma}\right)
$$

as $|s| \rightarrow \infty$. However the region of $\sigma$ where (5.2) is valid will depend on $I \in \mathcal{M}(M$ : $\vec{f}, \vec{p})$, because $\mathcal{M}(M: \vec{f}, \vec{p})$ may not be compact in general due to the splitting of trajectories, see Figure 5.1. However, as described in [Fu3], the compactification of $\mathcal{M}(M: \vec{f}, \vec{p})$ has only finitely many strata and the minimal stratum is compact. To effectively describe the non-compactness of $\mathcal{M}(M: \vec{f}, \vec{p})$, we introduce the variance of the energy, an analogue of which was previously used by Floer in the context of Floer homology (see [Lemma 2.1, F3]).

LEMMA 5.1. The function $V: \mathcal{M}(M: \vec{f}, \vec{p}) \rightarrow \mathbb{R}$ defined by

$$
V(I)=\left.\sum_{j=1}^{3} \frac{1}{2} \int_{-\infty}^{0} \tau^{2}|I|_{e_{i}}^{\prime}(\tau)\right|^{2} d \tau
$$

is everywhere defined and proper.

Proof. The integral converges for each $I \in \mathcal{M}$ because of the exponential decay of the gradient trajectories at nondegenerate critical points. Now, we prove the properness. It will be enough to prove that the set $V^{-1}([0, K])$ is compact for any $K>0$. Suppose the contrary. Since the non-compactness arises by the splitting of trajectories, there must exist a sequence $I^{k} \in V^{-1}([0, K])$ and $\tau_{k} \rightarrow \infty$ such that for some $j=1,2,3$, say $j=1$, the sequence of maps

$$
\left.\tau \mapsto I^{k}\right|_{e_{1}}\left(\tau-\tau_{k}\right)
$$




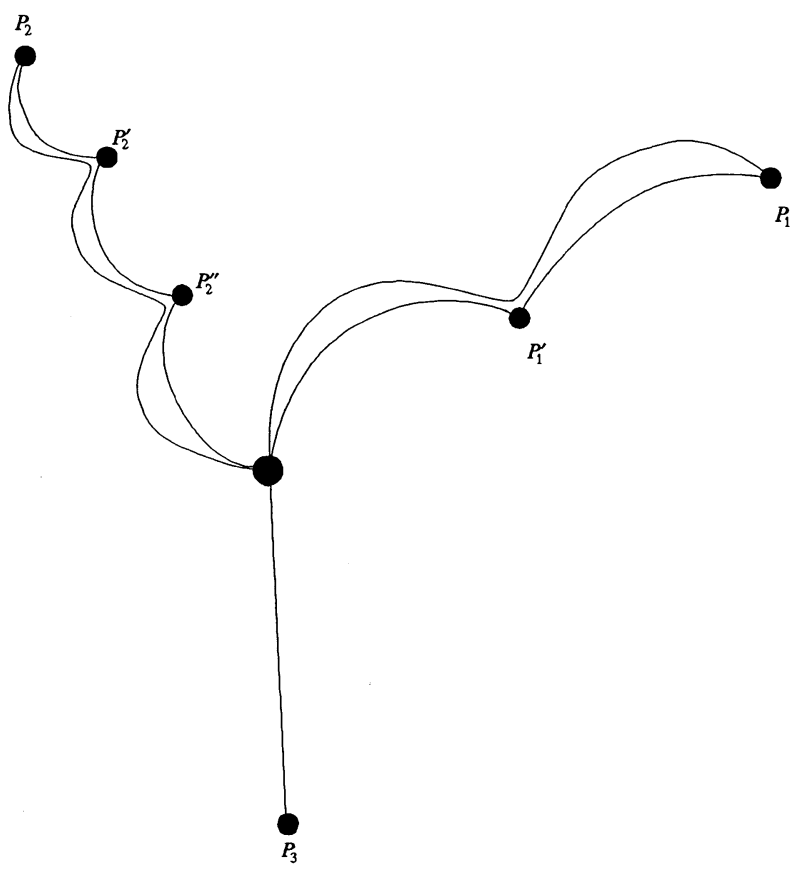

Figure 5.1

(locally) converges to a gradient trajectory $\chi: \mathbb{R} \rightarrow M$ of $f_{2}-f_{1}$. From this, it follows that the integral

$$
\left.\frac{1}{2} \int_{-\infty}^{0} \tau^{2}\left|\left(I^{k}\right)^{\prime}\right|_{e_{1}}(\tau)\right|^{2} d \tau=\left.\frac{1}{2} \int_{-\infty}^{\tau_{k}}\left(\tau-\tau_{k}\right)^{2}\left|\left(I^{k}\right)^{\prime}\right|_{e_{1}}\left(\tau-\tau_{k}\right)\right|^{2} d \tau
$$

goes to $+\infty$ as $k \rightarrow \infty$, which gives a contradiction to

$$
V\left(I_{k}\right) \leq K
$$

for all $k$.

It is obvious that

$$
\mathcal{M}(M: \vec{f}, \vec{p})=\bigcup_{K>0} V^{-1}([0, K])
$$

and we denote

$$
\mathcal{M}_{K}(M: \vec{f}, \vec{p})=V^{-1}([0, K])=\{I \in \mathcal{M}(M: \vec{f}, \vec{p}) \mid V(I) \leq K\} .
$$

By restricting to $\mathcal{M}_{K}$ for each $K>0$, we will have the uniform exponential decay at each triple $\vec{p}=\left(p_{1}, p_{2}, p_{3}\right)$ of critical points. More precisely, there exists

$$
R=R(K)>0
$$

such that we have

$$
\left|I^{\prime}\right|_{e_{j}}(\sigma) \mid \leq C e^{-C \sigma},
$$

for all $\sigma<-R, I \in \mathcal{M}_{K}$ and $j=1,2,3$. Therefore, we conclude

$$
\left\|\bar{\partial}_{J} w^{\epsilon}\right\|_{0, p, \epsilon, \Theta_{i}\left(\frac{2}{\epsilon^{2}}\right)}^{p} \leq C_{1}^{p}(K) \epsilon^{1+p}
$$


from (5.1) for all $I \in \mathcal{M}_{K}$, where

$$
C_{1}^{p}(K)=\widetilde{C}^{p} \sup _{I \in \mathcal{M}_{K}} \int_{0}^{\infty}\left|X_{\left(F_{i+1}-F_{i}\right)}\right|^{p}\left(\chi_{i}(\sigma)\right) d \sigma d t .
$$

Again for $I \in \mathcal{M}_{K}(M: \vec{f}, \vec{p})$, we now estimate $\left\|\bar{\partial}_{J} w^{\epsilon}\right\|_{0, p, \epsilon, \Theta_{0}\left(\frac{1}{\epsilon^{\alpha}}\right)}$. From the definition of $\bar{\partial}_{J}$, we have

$$
\begin{aligned}
\bar{\partial}_{J} w_{0}^{\epsilon} & =\bar{\partial}_{J} \exp _{x}\left(\epsilon \widetilde{w}_{0}\right) \\
& =\frac{D \exp _{x} \epsilon \widetilde{w}_{0}+J D \exp _{x} \in \widetilde{w}_{0} \circ i}{2} \\
& =\frac{D \exp _{x}\left(\epsilon \widetilde{w}_{0}\right) \circ \epsilon D \widetilde{w}_{0}+J D \exp _{x}\left(\epsilon \widetilde{w}_{0}\right) \circ \epsilon D \widetilde{w}_{0} \circ i}{2} \\
& =\epsilon D \exp _{x}\left(\epsilon \widetilde{w}_{0}\right)\left(\frac{D \widetilde{w}_{0}+\left(\exp _{x}\left(\epsilon \widetilde{w}_{0}\right)\right)^{*} J \circ D \widetilde{w}_{0} \circ i}{2}\right) \\
& =\epsilon D \exp _{x}\left(\epsilon \widetilde{w}_{0}\right)\left(\frac{\left(\exp _{x}\left(\epsilon \widetilde{w}_{0}\right)\right)^{*} J-J(x)}{2}\right) \circ D \widetilde{w}_{0} \circ i
\end{aligned}
$$

where we used the fact

$$
\bar{\partial}_{J_{0}} \widetilde{w}_{0}=0, \quad J_{0}=J(x)
$$

for the fourth identity. By the standard facts on the exponential map and the fact that $\left|\widetilde{w}_{0}(\tau, t)\right|$ grows linearly with respect to $|\tau| \rightarrow \infty$, we have

$$
\left|\left(\exp _{x}\left(\epsilon \widetilde{w}_{0}\right)\right)^{*} J-J_{0}\right| \leq C\left|\epsilon \widetilde{w}_{0}\right| \leq C \epsilon^{1-\alpha}
$$

on $\Theta_{i}\left(\frac{1}{\epsilon^{\alpha}}\right)$. Hence it follows

$$
\left|\bar{\partial}_{J} w_{0}^{\epsilon}\right| \leq C \epsilon^{2-\alpha}
$$

and so

$$
\begin{aligned}
& \left\|\bar{\partial}_{J} w_{0}^{\epsilon}\right\|_{0, p, \epsilon, \Theta_{0}\left(\frac{1}{\epsilon^{\alpha}}\right)}^{p}=\int_{\Theta_{0}\left(\frac{1}{\epsilon^{\alpha}}\right)} \epsilon^{2-p}\left|\bar{\partial}_{J} w_{0}\right|^{p} \\
& \quad \leq C^{p} \int_{\Theta_{0}\left(\frac{1}{\epsilon^{\alpha}}\right)} \epsilon^{2+p-\alpha p}=C^{p} \epsilon^{2+p-\alpha p} \text { Area }\left(\Theta_{0}\left(\frac{1}{\epsilon^{\alpha}}\right)\right) \\
& \quad \leq C_{2}^{p} \epsilon^{2+p-(p+1) \alpha}
\end{aligned}
$$

where the last inequality follows from that

$$
\operatorname{Area}\left(\Theta_{0}\left(\frac{1}{\epsilon^{\alpha}}\right)\right) \sim \frac{1}{\epsilon^{\alpha}}
$$

We note that the estimate (5.7) holds uniformly over all $I \in \mathcal{M}$ not just for $I \in \mathcal{M}_{K}$. Now, we need the estimates on the intermediate regions

$$
\Theta_{i}\left(\frac{1}{\epsilon^{\alpha}}, \frac{2}{\epsilon^{\alpha}}\right), \quad i=1,2,3
$$

Using the canonical coordinates on $\Theta_{i}$, we again estimate $\left|\frac{\partial w^{\epsilon}}{\partial \tau}+J \frac{\partial w^{\epsilon}}{\partial t}\right|$ instead of 
$\left|\bar{\partial}_{J} w^{\epsilon}\right|$. On $\Theta_{i}\left(\frac{1}{\epsilon^{\alpha}}, \frac{2}{\epsilon^{\alpha}}\right)$, we have

$$
\begin{aligned}
& \frac{\partial w^{\epsilon}}{\partial \tau}=\epsilon D \exp _{x}\left(\frac{\partial \widetilde{w}_{0}}{\partial \tau}+\epsilon^{\alpha} \beta^{\prime}\left(\epsilon^{\alpha} \tau\right)\left(\widetilde{w}_{i}^{\epsilon}-\widetilde{w}_{0}\right)+\beta\left(\epsilon^{\alpha} \tau\right)\left(\frac{\partial \widetilde{w}_{i}^{\epsilon}}{\partial \tau}-\frac{\partial \widetilde{w}_{0}}{\partial \tau}\right)\right) \\
& \frac{\partial w^{\epsilon}}{\partial t}=\epsilon D \exp _{x}\left(\frac{\partial \widetilde{w}_{i}}{\partial t}+\beta\left(\epsilon^{\alpha} \tau\right)\left(\frac{\partial \widetilde{w}_{i}^{\epsilon}}{\partial t}-\frac{\partial \widetilde{w}_{0}}{\partial t}\right)\right)
\end{aligned}
$$

Hence, from the equation $\frac{\partial \widetilde{w}_{0}}{\partial \tau}+J_{0} \frac{\partial \widetilde{w}_{0}}{\partial t}=0$,

$$
\begin{aligned}
\frac{\partial w^{\epsilon}}{\partial \tau}+J \frac{\partial w^{\epsilon}}{\partial t}= & \epsilon D \exp _{x}\left(\left(\exp _{x}^{*} J-J_{0}\right) \frac{\partial \widetilde{w}_{0}}{\partial t}\right)+\epsilon^{1+\alpha} D \exp _{x}\left(\beta^{\prime}\left(\epsilon^{\alpha} \tau\right)\left(\widetilde{w}_{i}^{\epsilon}-\widetilde{w}_{0}\right)\right) \\
+ & \epsilon \beta\left(\epsilon^{\alpha} \tau\right)\left\{\left(D \exp _{x}\left(\frac{\partial \widetilde{w}_{i}^{\epsilon}}{\partial \tau}\right)+J D \exp _{x}\left(\frac{\partial \widetilde{w}_{i}^{\epsilon}}{\partial t}\right)\right)\right. \\
& \left.-\left(D \exp _{x}\left(\frac{\partial \widetilde{w}_{0}}{\partial \tau}\right)+J D \exp _{x}\left(\frac{\partial \widetilde{w}_{0}}{\partial t}\right)\right)\right\}
\end{aligned}
$$

For the first term, we note as in (5.6)

$$
\left|\exp _{x}^{*} J-J_{0}\right| \leq C \mid \epsilon\left(\widetilde{w}_{0}(z)+\beta\left(\epsilon^{\alpha} \tau\right)\left(\widetilde{w}_{i}^{\epsilon}(z)-\widetilde{w}_{0}(z)\right) \mid .\right.
$$

From (4.2) and (4.3), we conclude

$$
\left|\widetilde{w}_{i}^{\epsilon}(z)-\widetilde{w}_{0}(z)\right|_{\Theta_{i}\left(\frac{1}{\epsilon^{\alpha}}, \frac{2}{\epsilon^{\alpha}}\right)}<C
$$

uniformly as $\epsilon \rightarrow 0$. Therefore, we have on $\Theta_{i}\left(\frac{1}{\epsilon^{\alpha}}, \frac{2}{\epsilon^{\alpha}}\right)$

$$
\left|\exp _{x}^{*} J-J_{0}\right| \leq C\left|\epsilon \widetilde{w}_{0}\right| \leq C \epsilon^{1-\alpha}
$$

and so

$$
\left|\epsilon D \exp _{x}\left(\left(\exp _{x}^{*} J-J_{0}\right) \frac{\partial \widetilde{w}_{0}}{\partial t}\right)\right| \leq C \epsilon^{2-\alpha} .
$$

For the second term in (5.8), we immediately have from (5.9)

$$
\left|\epsilon^{1+\alpha} D \exp _{x}\left(\beta^{\prime}\left(\epsilon^{\alpha} \tau\right)\left(\widetilde{w}_{i}^{\epsilon}-\widetilde{w}_{0}\right)\right)\right| \leq C \epsilon^{1+\alpha} .
$$

Therefore, we have from $(5.10)$

$$
\begin{gathered}
\int_{\Theta_{i}\left(\frac{1}{\epsilon^{\alpha}}, \frac{2}{\epsilon^{\alpha}}\right)} \epsilon^{2-p}\left|\epsilon D \exp _{x}\left(\left(\exp _{x}^{*} J-J_{0}\right) \frac{\partial \widetilde{w}_{0}}{\partial t}\right)\right|^{p} \\
\leq C^{p} \epsilon^{2+p-(p+1) \alpha}
\end{gathered}
$$

and from (5.11)

$$
\begin{aligned}
& \int_{\Theta_{i}\left(\frac{1}{\epsilon^{\alpha}}, \frac{2}{\epsilon^{\alpha}}\right)} \epsilon^{2-p}\left|\epsilon^{1+\alpha} D \exp _{x}\left(\beta^{\prime}\left(\epsilon^{\alpha} \tau\right)\left(\widetilde{w}_{i}^{\epsilon}-\widetilde{w}_{0}\right)\right)\right|^{p} \\
& \leq C^{p} \epsilon^{2+p \alpha-\alpha}=C^{p} \epsilon^{2+(p-1) \alpha}
\end{aligned}
$$

For the third term in (5.8), we consider two terms in the parenthesis separately. We first recall the definition of $\widetilde{w}_{i}^{\epsilon}$

$$
\widetilde{w}_{i}^{\epsilon}=\frac{1}{\epsilon}\left(\exp _{x}\right)^{-1} w_{i}^{\epsilon}
$$

and so

$$
w_{i}^{\epsilon}=\exp _{x}\left(\epsilon \widetilde{w}_{i}^{\epsilon}\right)
$$


We also have

$$
\begin{aligned}
D \exp _{x}\left(\frac{\partial \widetilde{w}_{i}^{\epsilon}}{\partial \tau}\right)+ & J D \exp _{x}\left(\frac{\partial \widetilde{w}_{i}^{\epsilon}}{\partial t}\right) \\
= & D \exp _{x}\left(\epsilon\left(\widetilde{w}_{0}(z)+\beta\left(\epsilon^{\alpha} \tau\right)\right)\left(\widetilde{w}_{i}^{\epsilon}(z)-\widetilde{w}_{0}(z)\right)\right)\left(\frac{\partial \widetilde{w}_{i}^{\epsilon}}{\partial \tau}\right) \\
& +J D \exp _{x}\left(\epsilon\left(\widetilde{w}_{0}(z)+\beta\left(\epsilon^{\alpha} \tau\right)\right)\left(\widetilde{w}_{i}^{\epsilon}(z)-\widetilde{w}_{0}(z)\right)\right)\left(\frac{\partial \widetilde{w}_{i}^{\epsilon}}{\partial t}\right) \\
= & \frac{1}{\epsilon}\left(\frac{\partial w_{i}^{\epsilon}}{\partial \tau}+J \frac{\partial w_{i}^{\epsilon}}{\partial t}\right) \\
& +\left(D \exp _{x} \epsilon\left(\widetilde{w}_{0}(z)+\beta\left(\epsilon^{\alpha} \tau\right)\right)\left(\widetilde{w}_{i}^{\epsilon}(z)-\widetilde{w}_{0}(z)\right)\right) \\
& -D \exp _{x}\left(\epsilon \widetilde{w}_{i}^{\epsilon}(z)\right)\left(\frac{\partial \widetilde{w}_{i}^{\epsilon}}{\partial \tau}\right) \\
& +\left(J D \operatorname { e x p } _ { x } \left(\epsilon \left(\widetilde{w}_{0}(z)+\beta\left(\epsilon^{\alpha} \tau\right)\left(\widetilde{w}_{i}^{\epsilon}(z)-\widetilde{w}_{0}(z)\right)\right.\right.\right. \\
& \left.-J D \exp _{x}\left(\epsilon \widetilde{w}_{i}^{\epsilon}(z)\right)\right)\left(\frac{\partial \widetilde{w}_{i}^{\epsilon}}{\partial t}\right)
\end{aligned}
$$

where we used the identity

$$
\begin{aligned}
& \frac{1}{\epsilon} \frac{\partial w_{i}^{\epsilon}}{\partial \tau}=D \exp _{x}\left(\epsilon \widetilde{w}_{i}^{\epsilon}(z)\right) \frac{\partial \widetilde{w}_{i}^{\epsilon}}{\partial \tau} \text { and } \\
& \frac{1}{\epsilon} \frac{\partial w_{i}^{\epsilon}}{\partial t}=D \exp _{x}\left(\epsilon \widetilde{w}_{i}^{\epsilon}(z)\right) \frac{\partial \widetilde{w}_{i}^{\epsilon}}{\partial t} .
\end{aligned}
$$

Therefore,

$$
\begin{aligned}
\epsilon \mid D \exp _{x}\left(\frac{\partial \widetilde{w}_{i}^{\epsilon}}{\partial \tau}\right) & +J D \exp _{x}\left(\frac{\partial \widetilde{w}_{i}^{\epsilon}}{\partial t}\right)|\leq| \frac{\partial w_{i}^{\epsilon}}{\partial \tau}+J \frac{\partial w_{i}^{\epsilon}}{\partial t} \mid \\
& +\epsilon \mid D \exp _{x}\left(\epsilon \left(\widetilde{w}_{0}(z)+\beta\left(\epsilon^{\alpha} \tau\right)\left(\widetilde{w}_{i}^{\epsilon}(z)-\widetilde{w}_{0}(z)\right)\right.\right. \\
& -D \exp _{x}\left(\epsilon \widetilde{w}_{i}^{\epsilon}(z)\right) \mid\left(\left|\frac{\partial \widetilde{w}_{i}^{\epsilon}}{\partial \tau}\right|+\left|\frac{\partial \widetilde{w}_{i}^{\epsilon}}{\partial t}\right|\right)
\end{aligned}
$$

However as in (5.4) one can estimate

$$
\int_{\Theta_{i}\left(\frac{1}{\epsilon^{\alpha}}, \frac{2}{\epsilon^{\alpha}}\right)}\left|\frac{\partial w_{i}^{\epsilon}}{\partial \tau}+J \frac{\partial w_{i}^{\epsilon}}{\partial t}\right|^{p} \leq C_{3}^{p}(K) \epsilon^{1+p}
$$

where $C_{3}(K)$ depends only on $K$. On the other hand, we have

$$
\begin{aligned}
& \epsilon \mid D \exp _{x}\left(\epsilon\left(\widetilde{w}_{0}(z)+\beta\left(\epsilon^{\alpha} \tau\right)\left(\widetilde{w}_{i}^{\epsilon}(z)-\widetilde{w}_{0}(z)\right)\right)-D \exp _{x}\left(\epsilon \widetilde{w}_{i}^{\epsilon}(z)\right) \mid\right. \\
& \quad \leq C \epsilon\left|D^{2} \exp _{x}\right| \cdot \mid \epsilon\left(1-\beta\left(\epsilon^{\alpha} \tau\right)\right)\left(\widetilde{w}_{i}^{\epsilon}(z)-\widetilde{w}_{0}(z) \mid\right. \\
& \quad \leq \widetilde{C} \epsilon^{2}\left|\widetilde{w}_{i}^{\epsilon}(z)-\widetilde{w}_{0}(z)\right| .
\end{aligned}
$$

Hence

$$
\begin{aligned}
& \int_{\Theta_{i}\left(\frac{1}{\epsilon^{\alpha}}, \frac{2}{\epsilon^{\alpha}}\right)} \epsilon^{2-p}\left(\epsilon \mid D \exp _{x}\left(\epsilon \left(\widetilde{w}_{0}(z)+\beta\left(\epsilon^{\alpha} \tau\right)\left(\widetilde{w}_{i}^{\epsilon}(z)-\widetilde{w}_{0}(z)\right)\right.\right.\right. \\
& \left.\quad-D \exp _{x}\left(\epsilon \widetilde{w}_{i}^{\epsilon}(z)\right) \mid\right)^{p}\left(\left|\frac{\partial \widetilde{w}_{i}^{\epsilon}}{\partial \tau}\right|+\left|\frac{\partial w_{i}^{\epsilon}}{\partial t}\right|\right)^{p} \\
& \leq \widetilde{C}^{p} \epsilon^{2+p} \int_{\Theta_{i}\left(\frac{1}{\epsilon^{\alpha}}, \frac{2}{\epsilon^{\alpha}}\right)}\left|\widetilde{w}_{i}^{\epsilon}(z)-\widetilde{w}_{0}(z)\right|^{p}\left(\left|\frac{\partial \widetilde{w}_{i}^{\epsilon}}{\partial \tau}\right|+\left|\frac{\partial \widetilde{w}_{i}^{\epsilon}}{\partial t}\right|\right)^{p} \\
& \leq C^{p} \epsilon^{2+p-\alpha}
\end{aligned}
$$


Combining (5.12), (5.13), (5.15) and (5.16), we have

$$
\begin{aligned}
\int_{\Theta_{i}\left(\frac{1}{\left.\epsilon^{\alpha}, \frac{2}{\epsilon^{\alpha}}\right)} \epsilon^{2-p}\right.}\left|\frac{\partial w^{\epsilon}}{\partial \tau}+J \frac{\partial w^{\epsilon}}{\partial t}\right|^{p} \\
\leq C^{p}(K)\left(\epsilon^{2+p-(p+1) \alpha}+\epsilon^{2+(p-1) \alpha}+\epsilon^{1+p}+\epsilon^{2+p-\alpha}\right)
\end{aligned}
$$

Using the fact

$$
0<\alpha<1
$$

we have obtained

$$
\int_{\Theta_{i}\left(\frac{1}{\epsilon^{\alpha}}, \frac{2}{\epsilon^{\alpha}}\right)} \epsilon^{p-2}\left|\frac{\partial w^{\epsilon}}{\partial \tau}+J \frac{\partial w^{\epsilon}}{\partial t}\right|^{p} \leq C_{4}^{p}(K) \epsilon^{2+(p-1) \alpha} .
$$

Now, summing up (5.4), (5.7) and (5.17), we have obtained

$$
\left\|\bar{\partial}_{J} w^{\epsilon}\right\|_{0, p, \epsilon}^{p} \leq\left(C_{1}^{p}(K) \epsilon^{1+p}+C_{2}^{p} \epsilon^{2+p-(p+1) \alpha}+C_{4}^{p}(K) \epsilon^{2+(p-1) \alpha}\right) .
$$

Hence, we have finally proved the following estimates.

Proposition 5.2. For each given $K>0$, the approximate solutions defined as in (4.9) satisfy the estimate

$$
\left\|\bar{\partial}_{J} w^{\epsilon}\right\|_{0, p, \epsilon} \leq C_{5} \epsilon^{\frac{2+(p-1) \alpha}{p}}
$$

for all $I \in \mathcal{M}_{K}(M: \vec{f}, \vec{p})$.

Now, we would like to extend the estimate (5.18) for all $I \in \mathcal{M}(M: \vec{f}, \vec{p})$ to prove the following main estimate of this section.

Proposition 5.3. There exists $\epsilon_{2}>0$ such that for $0<\epsilon<\epsilon_{2}$, the approximate solutions defined as in (4.9) satisfy the estimate

$$
\left\|\bar{\partial}_{J} w^{\epsilon}\right\|_{0, p, \epsilon} \leq C_{6} \epsilon^{\frac{2+(p-1) \alpha}{p}}
$$

for all $I \in \mathcal{M}$. In particular, we have

$$
\left\|\bar{\partial}_{J} w^{\epsilon}\right\|_{0, p, \epsilon} \rightarrow 0 \quad \text { as } \epsilon \rightarrow 0
$$

uniformly over $I \in \mathcal{M}=\mathcal{M}(M: \vec{f}, \vec{p})$.

Proof. It will be enough to have the estimate of the kind (5.19) for I's in $\mathcal{M} \backslash \mathcal{M}_{K}$ for sufficiently large $K>0$. We go back to the integral in (5.1)

$$
\int_{-\infty}^{0}\left|X_{\left(F_{i+1}-F_{i}\right)}\right|^{p}\left(\chi_{i}(\sigma)\right) d \sigma=\int_{-\infty}^{0}\left|X_{\left(F_{i+1}-F_{i}\right)}\right|^{p}\left(\left.I\right|_{e_{i}}(\sigma)\right) d \sigma .
$$

The following is a consequence of the standard concentration compactness principle whose proof we leave to readers.

LEMMA 5.4. Suppose that $\left\{I_{k}\right\} \subset \mathcal{M}(M: \vec{f}, \vec{p})$ be a sequence such that

$$
I_{k} \rightarrow I_{\infty}^{0}+\sum_{\ell=1}^{N} I_{\infty}^{\ell}
$$

in the weak topology, where $I_{\infty}^{0}$ is an element in $\mathcal{M}(M: \vec{f})$ and each $I_{\infty}^{\ell}$ is a gradient trajectory of $\left(f_{i+1}-f_{i}\right)$ for some $i=1,2,3$ connecting two critical points of $f_{i+1}-f_{i}$. 
Denote by

$$
W(I):=\sum_{i=1}^{3} \int_{-\infty}^{0}\left|X_{\left(F_{i+1}-F_{i}\right)}\right|^{p}\left(\left.I\right|_{e_{i}}(\sigma)\right) d \sigma
$$

for $I \in \mathcal{M}(M: \vec{f})$, and

$$
W(I):=\int_{-\infty}^{\infty}\left|X_{\left(F_{i+1}-F_{i}\right)}\right|^{p}(I(\sigma)) d \sigma
$$

for $I \in \mathcal{M}\left(f_{i+1}-f_{i}\right), i=1,2,3$. Then we have

$$
\lim _{k \rightarrow \infty} W\left(I_{k}\right)=W\left(I_{\infty}^{0}\right)+\sum_{\ell=1}^{N} W\left(I_{\infty}^{\ell}\right) .
$$

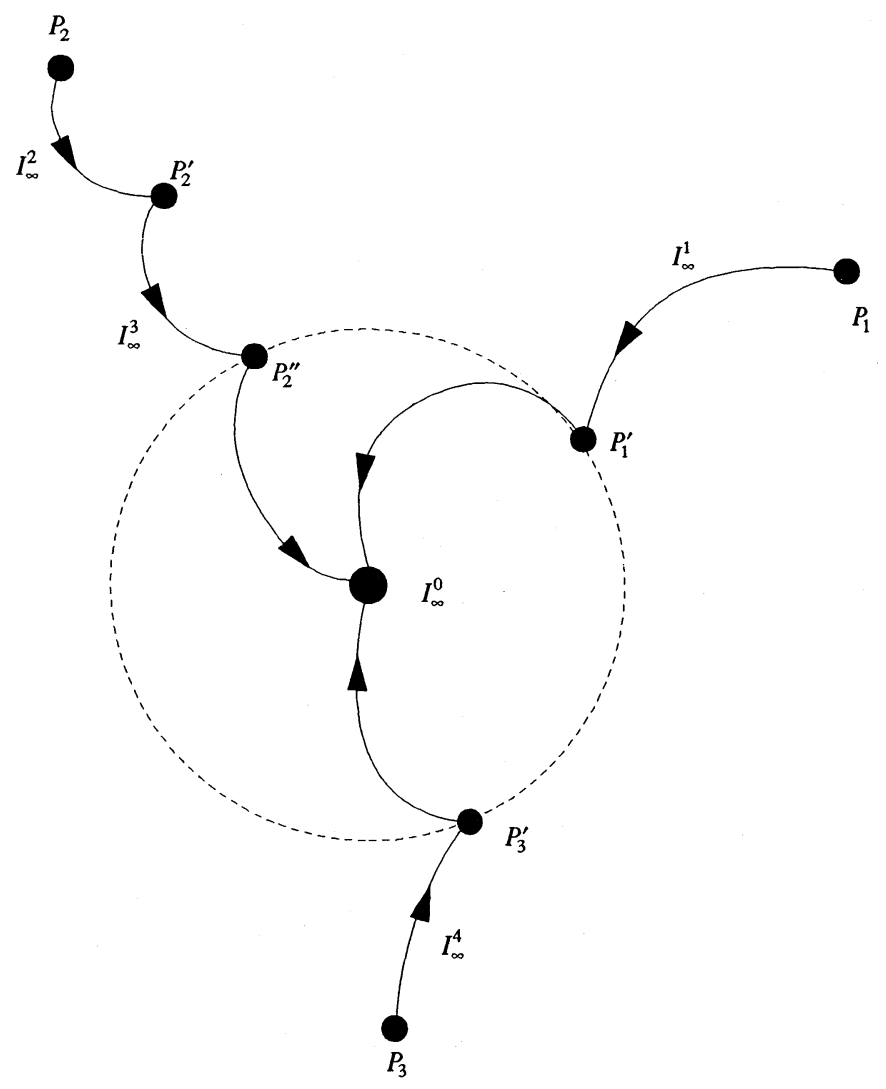

Figure 5.2

With this lemma, we proceed to the proof of Proposition 5.3. We recall that the compactification $\overline{\mathcal{M}}$ of $\mathcal{M}=\mathcal{M}(M: \vec{f}, \vec{p})$ has only finitely many strata $\overline{\mathcal{M}} \backslash \mathcal{M}$ and the minimal stratum, denoted by $\mathcal{M}^{0}$, is compact. We extend the definitions of $W$ to 
the whole compactification $\overline{\mathcal{M}}$ of $\mathcal{M}$ by defining

$$
W(I)=W\left(I^{0}\right)+\sum_{\ell=1}^{N} W\left(I^{\ell}\right)
$$

for $I=I^{0} \cup\left(\cup_{\ell=1}^{N} I^{\ell}\right)$ where we define

$$
W\left(I^{\ell}\right)=\int_{-\infty}^{\infty}\left|X_{\left(F_{i+1}-F_{i}\right)}\right|^{p}\left(I^{\ell}(\sigma)\right) d \sigma \quad \text { for } \ell=1, \cdots, N .
$$

By this definition, it follows from uniform exponential decay that $W$ is uniformly bounded on the minimal strata because they are compact. We denote by $R_{0}$ an upper bound of $W \mid \mathcal{M}^{0}$. Consider the stratum, denoted by $\mathcal{M}^{1}$, of the next higher order. Then we have

$$
\mathcal{M}^{0}=\overline{\mathcal{M}^{1}} \backslash \mathcal{M}^{1} .
$$

By the estimates similar to (5.4), (in fact easier than that), that for any fixed $K>0$, there exists a constant $R_{1}=R_{1}(K)>0, \epsilon_{1}>0$ such that

$$
W(I) \leq R_{1}
$$

for all $I \in \mathcal{M}_{K}^{1}$ and for all $0<\epsilon<\epsilon_{1}$.

On the other hand, Lemma 5.4 proves that for sufficiently large $K>0$, we have

$$
W(I) \leq R_{0}+\delta
$$

for some $\delta>0$ for all $I \in \mathcal{M}^{1} \backslash \mathcal{M}_{K}^{1}$. Combining (5.21) and (5.22), we have proven that there exists some $R_{2}>0$ such that

$$
W(I) \leq R_{2}
$$

for all $I \in \mathcal{M}^{1}$. By considering the stratum of next order and by repeating the above arguments, we finish the proof of Proposition 5.3.

6. Construction of the right inverse. We begin by rephrasing the transversality condition (3.2) of

$$
W_{p_{i}}^{-}\left(f_{i+1}-f_{i}\right) \quad i=1,2,3 .
$$

To simplify notations, we again denote

$$
\chi_{i}=\left.I\right|_{e_{i}}:(-\infty, 0] \rightarrow M
$$

for each

$$
I: T \rightarrow M, \quad I \in \mathcal{M}(M: \vec{f}, \vec{p}),
$$

and denote

$$
W_{\chi}^{k, p}:=W^{k, p}\left(\chi^{*} T M\right)
$$

which is the Sobolev space of the $W^{k, p}$-sections of $\chi^{*} T M$. We define

$$
\begin{aligned}
W_{I}^{k, p}:=\left\{\left(c_{\chi_{1}}, c_{\chi_{2}}, c_{\chi_{3}}\right) \in W_{\chi_{1}}^{k, p} \times W_{\chi_{2}}^{k, p} \times W_{\chi_{3}}^{k, p} \mid\right. \\
\left.c_{\chi_{1}}(0)=c_{\chi_{2}}(0)=c_{\chi_{3}}(0)\right\} .
\end{aligned}
$$

The space $W_{I}^{k, p}$ should be interpreted as a singular limit as $\epsilon \rightarrow 0$ of the spaces

$$
T_{\omega^{\epsilon}} \mathcal{F}_{\epsilon}^{k, p}=W_{\epsilon}^{k, p}\left(\left(w^{\epsilon}\right)^{*} T X\right) .
$$


We will restrict to $k=1$ from now on. Our transversality assumption (3.2) on $W_{p_{i}}^{-}\left(f_{i+1}-f_{i}\right)$ is equivalent to saying that the operator

$$
\begin{gathered}
L_{I}: W_{I}^{1, p} \rightarrow L_{\chi_{1}}^{p} \times L_{\chi_{2}}^{p} \times L_{\chi_{3}}^{p} \\
L_{I}\left(c_{\chi_{1}}, c_{\chi_{2}}, c_{\chi_{3}}\right):=\left(L_{\chi_{1}}\left(c_{\chi_{1}}\right), L_{\chi_{2}}\left(c_{\chi_{2}}\right), L_{\chi_{3}}\left(c_{\chi_{3}}\right)\right)
\end{gathered}
$$

is surjective, where the operator

$$
L_{\chi_{i}}: W_{\chi_{i}}^{1, p} \rightarrow L_{\chi_{i}}^{p}
$$

is defined to be the linearization operator

$$
L_{\chi_{i}}:=\nabla_{\tau}+\nabla \operatorname{grad}\left(f_{i+1}-f_{i}\right)
$$

of the equation

$$
\dot{\chi}+\operatorname{grad}\left(f_{i+1}-f_{i}\right)(\chi)=0 .
$$

By considering the $L^{2}$-adjoint of the operator $L_{I}$, it is also equivalent to the fact that the equation

$$
\left\{\begin{array}{l}
-\nabla_{\tau} c_{\chi_{i}}+\operatorname{grad}\left(f_{i+1}-f_{i}\right) c_{\chi_{i}}=0 \\
c_{\chi_{1}}(0)+c_{\chi_{2}}(0)+c_{\chi_{3}}(0)=0,
\end{array}\right.
$$

has only the trivial solution.

Now, we follow the strategy used in [F1] (or also see [MS]), i.e., first find an approximate right inverse

$$
Q_{\epsilon}: \widetilde{\mathcal{H}}_{w^{\epsilon}}^{p} \rightarrow W_{\epsilon}^{1, p}\left(\left(w^{\epsilon}\right)^{*} T X\right)
$$

of the operator $D_{w^{\epsilon}}:=D \bar{\partial}_{J}\left(w_{\epsilon}\right)$ for $\epsilon$ sufficiently small such that

$$
\left\|Q_{\epsilon}\right\| \leq C_{7},\left\|D_{w^{\epsilon}} \circ Q_{\epsilon}-\mathrm{id}\right\|<\frac{1}{2}
$$

where we recall that $\widetilde{\mathcal{H}}_{w^{\epsilon}}^{p}$ is defined as

$$
\widetilde{\mathcal{H}}_{w^{\epsilon}}^{p}=L_{\epsilon}^{p}\left(\Lambda^{(0,1)} T^{*} \Theta \otimes_{J}\left(w^{\epsilon}\right)^{*} T X\right) .
$$

Under these conditions, the composition

$$
D_{w^{\epsilon}} \circ Q_{\epsilon}: \tilde{\mathcal{H}}_{w^{\epsilon}}^{p} \rightarrow \tilde{\mathcal{H}}_{w^{\epsilon}}^{p}
$$

is invertible and a right inverse of $D_{w^{\epsilon}}$ will be given by

$$
Q_{w^{\epsilon}}:=Q_{\epsilon} \circ\left(D_{w^{\epsilon}} \circ Q_{\epsilon}\right)^{-1} \text {. }
$$

Now, we construct the approximate right inverse $Q_{\epsilon}$. We decompose $\Theta$ as before and describe the portion of $\xi=Q_{\epsilon}(\eta)$ on $\Theta_{i}\left(\frac{2}{\epsilon^{\alpha}}\right)$ first for each given $\eta \in \widetilde{\mathcal{H}}_{w^{\epsilon}}^{p}$. On $\Theta_{i}\left(\frac{2}{\epsilon^{\alpha}}\right)$, we use the coordinates $(\tau, t)$ and identify $\widetilde{\mathcal{H}}_{w^{\epsilon}}^{p}=L_{w^{\epsilon}}^{p}\left(\Lambda^{(0,1)} T^{*} \Theta \otimes_{J}\left(w^{\epsilon}\right)^{*} T X\right)$ with $L_{w^{\epsilon}}^{p}\left(w_{\epsilon}^{*} T X\right)$ in the standard way similar to the identification used in Section 4 . We recall that on $\Theta_{i}\left(\frac{2}{\epsilon^{\alpha}}\right), w^{\epsilon}$ was defined by

$$
w^{\epsilon}(\tau, t)=\phi_{\epsilon t}^{f_{i+1}} \circ \phi_{\epsilon t}^{f_{i}}\left(\chi_{i}(\epsilon \tau)\right)
$$

which can be rewritten as

$$
w^{\epsilon}(\tau, t)=\phi_{t}^{\epsilon f_{i+1}} \circ \phi_{t}^{\epsilon f_{i}}\left(\chi_{i}^{\epsilon}(\tau)\right), \quad \chi_{i}^{\epsilon}(\tau):=\chi_{i}(\epsilon \tau) .
$$

We note that $\chi_{i}^{\epsilon}$ is a trajectory of the gradient flow of $-\epsilon\left(f_{i+1}-f_{i}\right)$. 
Given $\eta \in \widetilde{\mathcal{H}}_{w^{\epsilon}}^{p} \cong L_{\epsilon}^{p}\left(\left(w^{\epsilon}\right)^{*} T X\right)$, we define the triple

$$
\vec{b}_{\chi}^{\epsilon}=\left(b_{1}^{\epsilon}, b_{2}^{\epsilon}, b_{3}^{\epsilon}\right)
$$

by

$$
b_{i}^{\epsilon}(\tau, t)= \begin{cases}T\left(\phi^{f_{i+1}^{\epsilon t}} \circ \phi_{\epsilon(1-t)}^{f_{i}}\right)^{-1} \eta(\tau, t) & \text { for } \tau \leq-\frac{3}{2 \epsilon^{\alpha}} \\ 0 & \text { otherwise }\end{cases}
$$

Since $w^{\epsilon}(\tau, t)=\phi_{\epsilon t}^{f_{i+1}} \circ \phi_{\epsilon(1-t)}^{f_{i}}\left(\chi_{i}(\epsilon \tau)\right)$, we have

$$
b_{i}^{\epsilon} \in L_{\epsilon}^{p}\left(\left(\chi_{i}^{\epsilon}\right)^{*} T X \times[0,1]\right):=\tilde{L}_{\chi_{i}}^{p}
$$

where $\chi_{i}^{\epsilon}(\tau)=\chi_{i}(\epsilon \tau)$. We will now study the following equation in detail in the proof of Proposition 6.1 below:

$$
\left\{\begin{array}{l}
\nabla_{\tau} a_{i}^{\epsilon}+J\left(\nabla_{t}+\epsilon \nabla X_{\left(F_{i+1}-F_{i}\right)}\right) a_{i}^{\epsilon}=b_{i}^{\epsilon} \\
a_{i}^{\epsilon}(\tau, 0), a_{i}^{\epsilon}(\tau, 1) \subset T M \subset T X \quad \text { and } \quad a_{i}^{\epsilon}(0, t) \in T M \\
\int_{0}^{1} a_{1}^{\epsilon}(0, t) d t=\int_{0}^{1} a_{2}^{\epsilon}(0, t) d t=\int_{0}^{1} a_{3}^{\epsilon}(0, t) d t .
\end{array}\right.
$$

We define, for each $i=1,2,3$,

$$
\widetilde{W}_{\chi_{i}^{\epsilon}}^{1, p}:=\left\{\vec{a}^{\epsilon} \in W^{1, p}\left(\left(\chi_{i}^{\epsilon}\right)^{*} T X \times[0,1]\right) \mid a_{i}^{\epsilon}(\tau, 0), a_{i}^{\epsilon}(\tau, 1) \in T M \subset T X\right\}
$$

and

$$
\begin{array}{r}
\widetilde{W}_{I^{\epsilon}}^{1, p}:=\left\{\left(a_{1}^{\epsilon}, a_{2}^{\epsilon}, a_{3}^{\epsilon}\right) \in \widetilde{W}_{\chi_{i}^{\epsilon}}^{1, p} \times \widetilde{W}_{\chi_{i}^{\epsilon}}^{1, p} \times \widetilde{W}_{\chi_{i}^{\epsilon}}^{1, p} \mid a_{i}^{\epsilon}(0, t) \in T_{x} M \subset T_{x} X\right. \\
\text { and } \left.\int_{0}^{1} a_{1}^{\epsilon}(0, t) d t=\int_{0}^{1} a_{2}^{\epsilon}(0, t) d t=\int_{0}^{1} a_{3}^{\epsilon}(0, t) d t\right\}
\end{array}
$$

We equip $\widetilde{W}_{I^{\epsilon}}^{1, p}$ with the norm $\|\cdot\|_{1, p, \epsilon}$. Similarly we define

$$
\widetilde{L}_{I^{\epsilon}}^{p}:=\widetilde{L}_{\chi_{1}}^{p} \times \widetilde{L}_{\chi_{2}}^{p} \times \widetilde{L}_{\chi_{3}}^{p}
$$

and equip it with the norm $\|\cdot\|_{0, p, \epsilon}$. Now consider the operator

$$
\widetilde{D}_{I^{\epsilon}}: \widetilde{W}_{I^{\epsilon}}^{1, p} \rightarrow \widetilde{L}_{I^{\epsilon}}^{p}
$$

by

$$
\tilde{D}_{I^{\epsilon}}\left(a_{1}^{\epsilon}, a_{2}^{\epsilon}, a_{3}^{\epsilon}\right)=\left(\widetilde{D}_{\chi_{1}^{\epsilon}} a_{1}^{\epsilon}, \widetilde{D}_{\chi_{2}^{\epsilon}} a_{2}^{\epsilon}, \widetilde{D}_{\chi_{2}^{\epsilon}} a_{2}^{\epsilon}\right)
$$

where

$$
\widetilde{D}_{\chi_{i}^{\epsilon}} a_{i}^{\epsilon}:=\nabla_{\tau} a_{i}^{\epsilon}+J\left(\nabla_{t}+\epsilon \nabla X_{\left(F_{i+1}-F_{i}\right)}\right) a_{i}^{\epsilon} .
$$

Proposition 6.1. Suppose that $W_{p_{i}}^{-}\left(f_{i+1}-f_{i}\right)$ for $i=1,2,3$ intersect transversely and so the equation (6.1) has no non-trivial solution. Then there exists $\epsilon_{2}>0$ such that if $0<\epsilon<\epsilon_{2}$, the following hold:

(i).

Ker $\widetilde{D}_{I^{\epsilon}}=\left\{\left(a_{1}^{\epsilon}, a_{2}^{\epsilon}, a_{3}^{\epsilon}\right) \in \widetilde{W}_{I^{\epsilon}}^{1, p} \mid a_{i}^{\epsilon}\right.$ 's are independent of $t$ and satisfy the equation $\left.\nabla_{\tau} a_{i}^{\epsilon}+\epsilon \nabla \operatorname{grad}\left(f_{i+1}-f_{i}\right) a_{i}^{\epsilon}=0\right\}$

(ii). $\widetilde{D}_{I^{\epsilon}}$ is surjective and 
(iii). there exists $C_{8}>0$ independent of $\epsilon$ such that for any $\vec{a}^{\epsilon}=\left(a_{1}^{\epsilon}, a_{2}^{\epsilon}, a_{3}^{\epsilon}\right) \epsilon$ $\left(\operatorname{Ker} \widetilde{D}_{I^{\epsilon}}\right)^{\perp} \subset \widetilde{W}_{I^{\epsilon}}^{1, p}$, we have

$$
\left\|\vec{a}^{\epsilon}\right\|_{1, p, \epsilon} \leq C_{8}\left\|\tilde{D}_{I^{\epsilon}}\left(\vec{a}^{\epsilon}\right)\right\|_{0, p, \epsilon}
$$

and so that there exists a right inverse $\widetilde{Q}_{I^{\epsilon}}$ of $\widetilde{D}_{I^{\epsilon}}$ such that

$$
\widetilde{D}_{I^{\epsilon}} \circ \widetilde{Q}_{I^{\epsilon}}=i d \text { and }\left\|\widetilde{Q}_{I^{\epsilon}}\right\| \leq C_{8} .
$$

Proof. We separate the proof into 3 parts.

Proof of (i). Suppose that

$$
\vec{a}^{\epsilon}=\left(a_{1}^{\epsilon}, a_{2}^{\epsilon}, a_{3}^{\epsilon}\right) \in \operatorname{Ker} \widetilde{D}_{I^{\epsilon}} \subset \widetilde{W}_{I^{\epsilon}}^{1, p}
$$

i.e, satisfies the equation

$$
\left\{\begin{array}{l}
\nabla_{\tau} a_{i}^{\epsilon}+J\left(\nabla_{t}+\epsilon \nabla X_{\left(F_{i+1}-F_{i}\right)}\right) a_{i}^{\epsilon}=0 \\
a_{i}^{\epsilon}(\tau, 0), a_{i}^{\epsilon}(\tau, 1) \in T M \subset T X \text { and } a_{i}^{\epsilon}(0, t) \in T M \\
\int_{0}^{1} a_{1}^{\epsilon}(0, t) d t=\int_{0}^{1} a_{2}^{\epsilon}(0, t) d t=\int_{0}^{1} a_{3}^{\epsilon}(0, t) d t \in T M .
\end{array}\right.
$$

Following the idea in [F2] and [Appendix, O6], we decompose

$$
a_{i}^{\epsilon}=c_{i}^{\epsilon}+d_{i}^{\epsilon}
$$

where $c_{i}^{\epsilon}\left(\right.$ resp. $\left.d_{i}^{\epsilon}\right)$ is the horizontal (resp. vertical) component of $\left(I^{\epsilon}\right)^{*} T\left(T^{*} M\right)$ in terms of the splitting

$$
\left.T\left(T^{*} M\right)\right|_{M}=T M \oplus T^{*} M .
$$

Then $\left(c_{i}^{\epsilon}, d_{i}^{\epsilon}\right)$ must satisfy the equation

$$
\left\{\begin{array}{l}
\nabla_{\tau} c_{i}^{\epsilon}+\nabla_{t} d_{i}^{\epsilon}+\epsilon \nabla \operatorname{grad}\left(f_{i+1}-f_{i}\right) c_{i}^{\epsilon} \\
\nabla_{\tau} d_{i}^{\epsilon}-\nabla_{t} c_{i}^{\epsilon}=0 \\
d_{i}^{\epsilon}(\tau, 0)=0, d_{i}^{\epsilon}(\tau, 1)=0 \\
d_{1}^{\epsilon}(0, t)=d_{2}^{\epsilon}(0, t)=d_{3}^{\epsilon}(0, t)=0 \\
\int_{0}^{1} c_{1}^{\epsilon}(0, t) d t=\int_{0}^{1} c_{2}^{\epsilon}(0, t) d t=\int_{0}^{1} c_{3}^{\epsilon}(0, t) d t .
\end{array}\right.
$$

Here we identify $\nabla_{(*)} d_{i}^{\epsilon} \in V T_{I^{\epsilon}}\left(T^{*} M\right) \cong T_{I^{\epsilon}}^{*} M$ with $J\left(\nabla_{(*)} d_{i}^{\epsilon}\right) \in T_{I^{\epsilon}} M$ using the canonical decomposition of $\left.T\left(T^{*} M\right)\right|_{M}$. We now consider the function

$$
\beta_{i}^{\epsilon}=\frac{1}{2}\left\langle d_{i}^{\epsilon}(\tau), d_{i}^{\epsilon}(\tau)\right\rangle
$$

and then a straightforward computation using the boundary condition (6.9) yields

$$
\frac{d^{2} \beta_{i}^{\epsilon}}{d \tau^{2}}=\left\|\nabla_{\tau} d_{i}^{\epsilon}\right\|^{2}+\left\|\nabla_{t} d_{i}^{\epsilon}\right\|^{2}-\epsilon\left\langle d_{i}^{\epsilon}(\tau), \nabla \operatorname{grad}\left(f_{i+1}-f_{i}\right) \nabla_{\tau} d_{i}^{\epsilon}\right\rangle .
$$

(See [Appendix, O6] for this computation.) Again using (6.9) and the Poincaré inequality, we have

$$
\left\|d_{i}^{\epsilon}\right\|_{2} \leq C\left\|\nabla_{t} d_{i}^{\epsilon}\right\|_{2}
$$

Therefore we have

$$
\frac{d^{2} \beta_{i}^{\epsilon}}{d \tau^{2}} \geq\left\|\nabla_{\tau} d_{i}^{\epsilon}\right\|^{2}+\left\|\nabla_{t} d_{i}^{\epsilon}\right\|^{2}-C \epsilon\left\|\nabla \operatorname{grad}\left(f_{i+1}-f_{i}\right)\right\|_{\infty}\left\|\nabla_{t} d_{i}^{\epsilon}\right\|_{2}\left\|\nabla_{\tau} d_{i}^{\epsilon}\right\|_{2}
$$


and so if we choose $\epsilon$ so that

$$
C \epsilon\left\|\nabla \operatorname{grad}\left(f_{i+1}-f_{i}\right)\right\|_{\infty} \leq \frac{1}{4}
$$

we get

$$
\frac{d^{2} \beta_{i}^{\epsilon}}{d \tau^{2}} \geq \frac{1}{2}\left\|\nabla_{t} d_{i}^{\epsilon}\right\|_{2}^{2} \geq \frac{1}{2 C^{2}}\left\|d_{i}^{\epsilon}\right\|^{2}=\frac{1}{2 C^{2}} \beta_{i}^{\epsilon}>0
$$

which shows that $\beta_{i}^{\epsilon}$ is a convex function. Since $\vec{a}^{\epsilon} \in \widetilde{W}_{I^{\epsilon}}^{1, p}$, we have

$$
\lim _{\tau \rightarrow-\infty} \beta_{i}^{\epsilon}(\tau)=0
$$

and (6.10) implies

$$
\beta_{i}^{\epsilon}(0)=0 .
$$

We fix any $\epsilon$ satisfying (6.12) so that $\beta_{i}^{\epsilon}$ becomes a convex function. Then (6.13), (6.14) and the convexity of $\beta_{i}^{\epsilon}$ together imply that $\beta_{i}^{\epsilon} \equiv 0$ which in turn proves $d_{i}^{\epsilon} \equiv 0$. Then this and (6.7) imply that $c_{i}^{\epsilon}$ is $t$-independent and it satisfies the equation

$$
\nabla_{\tau} c_{i}^{\epsilon}+\epsilon \nabla \operatorname{grad}\left(f_{i+1}-f_{i}\right) c_{i}^{\epsilon}=0 .
$$

This together with (6.11) proves (i).

Proof of (ii). To prove the surjectivity, it is enough to prove

$$
\text { Coker } \widetilde{D}_{I^{\epsilon}}=\{0\} \text {. }
$$

Using the $L^{2}$-inner product, we first derive the $L^{2}$-adjoint equation of (6.6). The $L^{2}$-cokernel element is characterized by the condition

$$
\begin{aligned}
0 & =\left\langle\widetilde{D}_{I^{\epsilon}} \vec{a}^{\epsilon}, \vec{b}^{\epsilon}\right\rangle \\
& =\sum_{j=1}^{3} \int_{-\infty}^{0} \int_{0}^{1}\left\langle\nabla_{\tau} a_{i}^{\epsilon}+J\left(\nabla_{t}+\epsilon \nabla X_{\left(F_{i+1}-F_{i}\right)}\right) a_{i}^{\epsilon}, b_{i}^{\epsilon}\right\rangle
\end{aligned}
$$

for any $\vec{a}^{\epsilon}=\left(a_{1}^{\epsilon}, a_{2}^{\epsilon}, a_{3}^{\epsilon}\right) \in \widetilde{W}_{I^{\epsilon}}^{1, p}$. Since $b_{i}^{\epsilon}$ will be smooth (by elliptic regularity!), a simple computation by integration by parts, using the fact that $J$ is parallel along $M \subset T^{*} M$, shows that $\vec{b}^{\epsilon}$ satisfies the equation

$$
\left\{\begin{array}{l}
\left.-\nabla_{\tau} b_{i}^{\epsilon}+J\left(\nabla_{t}+\epsilon \nabla X_{\left(F_{i+1}-F_{i}\right)}\right)\right) b_{i}^{\epsilon}=0 \\
b_{i}^{\epsilon}(\tau, 0), b_{i}^{\epsilon}(\tau, 1) \in T M \subset T X \\
\left(b_{i}^{\epsilon}\right)^{\|}(0, \cdot) \text { are independent of } t \text { and } \sum_{i=1}^{3}\left(b_{i}^{\epsilon}\right)^{\|}(0, t) \equiv 0 .
\end{array}\right.
$$

where $b_{i}^{\epsilon} \|$ is the horizontal component of $b_{i}^{\epsilon}$. As before, we decompose $b_{i}^{\epsilon}=\left(e_{i}^{\epsilon}, f_{i}^{\epsilon}\right) \epsilon$ $T X \cong T M \oplus T^{*} M$ and then $\left(e_{i}^{\epsilon}, f_{i}^{\epsilon}\right)$ satisfies

$$
\left\{\begin{array}{l}
-\nabla_{\tau} e_{i}^{\epsilon}+\nabla_{t} f_{i}^{\epsilon}+\epsilon \nabla \operatorname{grad}\left(f_{i+1}-f_{i}\right) f_{i}^{\epsilon}=0 \\
-\nabla_{\tau} f_{i}^{\epsilon}-\nabla_{t} e_{i}^{\epsilon}=0 \\
f_{i}^{\epsilon}(\tau, 0)=0, f_{i}^{\epsilon}(\tau, 1)=0 \\
e_{i}^{\epsilon}(0, \cdot) \text { are independent of } t \text { and } e_{1}^{\epsilon}+e_{2}^{\epsilon}+e_{3}^{\epsilon}=0
\end{array}\right.
$$

Again we consider the function

$$
\gamma_{i}^{\epsilon}(\tau)=\frac{1}{2}\left\langle f_{i}^{\epsilon}(\tau), f_{i}^{\epsilon}(\tau)\right\rangle
$$


and then $\gamma_{i}^{\epsilon}$ can be shown to satisfy as before

$$
\begin{aligned}
& \frac{d^{2} \gamma_{i}^{\epsilon}}{d \tau^{2}} \geq \frac{1}{2 C^{2}} \gamma_{i}^{\epsilon} \\
& \lim _{\tau \rightarrow-\infty} \gamma_{i}^{\epsilon}=0 .
\end{aligned}
$$

Since $e_{i}^{\epsilon}(0, \cdot)$ are independent of $t$ from $(6.18), \nabla_{t} e_{i}^{\epsilon}(0, t) \equiv 0$ which in turn implies by (6.16)

$$
\nabla_{\tau} f_{i}^{\epsilon}(\tau, 0) \equiv 0
$$

Therefore we have

$$
\frac{d \gamma_{i}^{\epsilon}}{d \tau}(\tau, 0)=\left\langle f_{i}^{\epsilon}(\tau, 0), \nabla_{\tau} f_{i}^{\epsilon}(\tau, 0)\right\rangle \equiv 0
$$

Combining (6.19), (6.20) and (6.21), we conclude (by strong maximum principle!) $\gamma_{i}^{\epsilon} \equiv 0$ and hence

$$
f_{i}^{\epsilon} \equiv 0 \text {. }
$$

Substitution of this into (6.14) proves that $e_{i}^{\epsilon}$ satisfies

$$
\left\{\begin{array}{l}
-\nabla_{\tau} e_{i}^{\epsilon}+\epsilon \nabla \operatorname{grad}\left(f_{i+1}-f_{i}\right) e_{i}^{\epsilon}=0 \\
e_{1}^{\epsilon}(0)+e_{2}^{\epsilon}(0)+e_{3}^{\epsilon}(0)=0
\end{array}\right.
$$

Therefore if we re-scale $e_{i}^{\epsilon}$ and define

$$
\widetilde{e}_{i}^{\epsilon}(\sigma)=e_{i}^{\epsilon}\left(\frac{\sigma}{\epsilon}\right)
$$

$\tilde{e}_{i}^{\epsilon}$ will satisfy

$$
\left\{\begin{array}{l}
-\nabla_{\sigma} \widetilde{e}_{i}^{\epsilon}+\nabla \operatorname{grad}\left(f_{i+1}-f_{i}\right) \widetilde{e}_{i}^{\epsilon}=0 \\
\widetilde{e}_{1}^{\epsilon}(0)+\widetilde{e}_{2}^{\epsilon}(0)+\widetilde{e}_{3}^{\epsilon}(0)=0 .
\end{array}\right.
$$

Now the transversality hypothesis that (6.1) has only the trivial solution implies that

$$
\widetilde{e}_{i}^{\epsilon} \equiv 0 \quad \text { and hence } \quad e_{i}^{\epsilon} \equiv 0 .
$$

Now (6.22) and (6.23) show that Coker $\widetilde{D}_{I^{\epsilon}}=0$ and so prove the surjectivity.

Proof of (iii). We may assume without loss of any generality, by replacing $f_{i}$ by $\epsilon_{2} f_{i}$ in (i) and (ii), that $\epsilon_{2}=1$. Then (i) and (ii) implies the estimate

$$
\left\|\vec{a}^{1}\right\|_{1, p} \leq C_{8}\left\|\widetilde{D}_{I} \vec{a}^{1}\right\|_{0, p}
$$

for all $\vec{a}^{1} \in\left(\operatorname{Ker} \widetilde{D}_{I}\right)^{\perp} \subset \widetilde{W}_{I}^{1, p}$. It would be enough to prove (6.4) for

$$
\epsilon=\frac{1}{2^{k}}, \text { for each nonnegative integer } k \text {. }
$$

To prove this, we define

$$
\tilde{a}^{\epsilon}(\sigma, s)=a^{\epsilon}\left(\frac{\sigma}{\epsilon}, \frac{s}{\epsilon}\right) \quad \text { for } 0 \leq s \leq \frac{1}{2^{k}},-\infty \leq \sigma \leq 0 .
$$

Now we extend $\tilde{a}^{\epsilon}$ by reflection to $0 \leq s \leq \frac{1}{2^{k-1}}$ : Under the decomposition $a^{\epsilon}=b^{\epsilon}+c^{\epsilon}$, the conjugation with respect to the canonical almost complex structure is nothing but the linear map $a^{\epsilon}=b^{\epsilon}+c^{\epsilon} \mapsto b^{\epsilon}-c^{\epsilon}$. Then we define

$$
\tilde{a}^{\epsilon}(\sigma, t)=\tilde{b}^{\epsilon}(\sigma, t)-\widetilde{c}^{\epsilon}\left(\sigma, \frac{1}{2^{k-1}}-t\right) \quad \text { for } \quad \frac{1}{2^{k}} \leq t \leq \frac{1}{2^{(k-1)}} .
$$


After then we extend this to the whole $t \in[0,1]$ in an obvious way, which we again denote by $\tilde{a}=\tilde{a}(\sigma, t)$. From the construction, it is easy to check that $\tilde{a} \in \widetilde{W}_{I}^{1, p}$. It is now crucial to observe that since we have proven in (i) that the elements in $\operatorname{Ker} \widetilde{D}_{I^{\epsilon}}$ are independent of $t$, the extension $\widetilde{a}^{\epsilon}$ can be easily proven to be still in $\left(\operatorname{Ker} \widetilde{D}_{I}\right)^{\perp}$. Therefore we have the estimate

$$
\left\|\widetilde{a}^{\epsilon}\right\|_{1, p} \leq C_{8}\left\|\widetilde{D}_{I} \widetilde{a}^{\epsilon}\right\|_{0, p}
$$

from (6.24). However by the periodicity of $\tilde{a}^{\epsilon}$, this implies

$$
\left\|\widetilde{a}^{\epsilon}\right\|_{1, p, 0 \leq t \leq \epsilon} \leq C_{8}\left\|\widetilde{D}_{I} \widetilde{a}^{\epsilon}\right\|_{0, p, 0 \leq t \leq \epsilon} .
$$

Scaling back to $(\tau, t) \in(-\infty, 0] \times[0,1]$, this is equivalent to the required estimate (6.4). This finally finishes the proof of Proposition 6.1.

We now proceed the construction of $Q_{\epsilon}$. For each given $\eta \in \widetilde{\mathcal{H}}_{w^{\epsilon}}^{p}$, we define $\vec{b}^{\epsilon}=\left(b_{1}^{\epsilon}, b_{2}^{\epsilon}, b_{3}^{\epsilon}\right)$ on $\Theta \backslash \Theta_{0}\left(\frac{2}{\epsilon^{\alpha}}\right)$ as in (6.3) and apply the operator $\widetilde{Q}_{I^{\epsilon}}$ to $\vec{b}^{\epsilon}$ to define

$$
\vec{a}^{\epsilon}=\left(a_{1}^{\epsilon}, a_{2}^{\epsilon}, a_{3}^{\epsilon}\right) \in \widetilde{W}_{I^{\epsilon}}^{1, p}
$$

by

$$
\vec{a}^{\epsilon}=\widetilde{Q}_{I^{\epsilon}}\left(\vec{b}^{\epsilon}\right)
$$

In particular, we have

$$
b_{i}^{\epsilon}=\tilde{D}_{\chi_{i}^{\epsilon}}\left(a_{i}^{\epsilon}\right)=\nabla_{\tau} a_{i}^{\epsilon}+J\left(\nabla_{t}+\epsilon \nabla X_{\left(F_{i+1}-F_{i}\right)}\right) a_{i}^{\epsilon}
$$

and so by definition of $b_{i}^{\epsilon}$ in (6.3),

$$
\nabla_{\tau} a_{i}^{\epsilon}+J\left(\nabla_{t}+\epsilon \nabla X_{\left(F_{i+1}-F_{i}\right)}\right) a_{i}^{\epsilon}=0
$$

for $-\frac{3}{2 \epsilon^{\alpha}}<\tau \leq 0$ and

$$
\begin{aligned}
a_{i}^{\epsilon}(0, t) & \in T_{x} M \subset T_{x} X \quad \text { and } \\
\int_{0}^{1}\left(a_{1}^{\epsilon}\right)(0, t) d t & =\int_{0}^{1}\left(a_{2}^{\epsilon}\right)(0, t) d t=\int_{0}^{1}\left(a_{3}^{\epsilon}\right)(0, t) d t \in T_{x} M
\end{aligned}
$$

where we recall

$$
\chi_{1}(0)=\chi_{2}(0)=\chi_{3}(0)=x \quad \text { and } \quad a_{i}^{\epsilon}(0, t) \in T_{x} X .
$$

Using, $\vec{a}^{\epsilon}$, we now define

$$
\xi(\tau, t)=T\left(\phi_{\epsilon t}^{f_{i+1}} \circ \phi_{\epsilon(1-t)}^{f_{i}}\right)\left(a_{i}^{\epsilon}(\tau, t)\right)
$$

on $\Theta_{i}\left(\frac{2}{\epsilon^{\alpha}}\right)$ for $i=1,2,3$.

We next describe the portion of $\xi$ restricted to $\left.\xi\right|_{\Theta_{0}\left(\frac{1}{\epsilon^{\alpha}}\right)}$. As before, we identify $\left(T_{x} X, J(x)\right)$ with $\left(\mathbb{C}^{n}, J_{0}\right)$ and consider the linearization of $\bar{\partial}$ :

$$
D_{\widetilde{w}} \bar{\partial}: W^{1, p}\left(\widetilde{w}_{0}^{*} T \mathbb{C}^{n}\right) \rightarrow L^{p}\left(\Omega^{(0,1)} \widetilde{w}_{0}^{*} T \mathbb{C}^{n}\right)
$$


LEMMA 6.1. The linearization operator $D_{\widetilde{w}_{\epsilon}} \bar{\partial}$ in (6.29) is invertible.

Proof. First note that the kernel element of $D_{\widetilde{w}_{0}} \bar{\partial}$ is described by the equation

$$
\left\{\begin{array}{l}
\bar{\partial} \xi=0 \\
\xi\left(\ell_{i}\right) \subset T \tilde{\Lambda}_{i} \cong \mathbb{R}^{n} \\
\|\xi\|_{1, p}<\infty
\end{array}\right.
$$

from which it follows by the maximum principle that

$$
\operatorname{Ker} D_{\widetilde{w}_{0}} \bar{\partial}=\{0\} \text {. }
$$

Next, we prove

$$
\text { Coker } D_{\widetilde{w}_{0}} \bar{\partial}=\{0\}
$$

which will finish the proof. Using the $L^{2}$-inner product, one can identify the dual of $L^{p}\left(\Omega^{(0,1)}\left(\widetilde{w}_{0}^{*} T \mathbb{C}^{n}\right)\right)$ with

$$
L^{q}\left(\Omega^{(1,0)}\left(\widetilde{w}_{0}^{*} T \mathbb{C}^{n}\right)\right), \quad \frac{1}{p}+\frac{1}{q}=1 .
$$

Then the element $\eta \in L^{q}\left(\Omega^{(1,0)}\left(\widetilde{w}_{0}^{*} T \mathbb{C}^{n}\right)\right)$ which is in Coker $D_{\widetilde{w}_{0}} \bar{\partial}$ is characterized by the equation

$$
\left\{\begin{array}{l}
\operatorname{Re} \int_{\Theta}\langle\bar{\partial} \xi, \eta\rangle=0 \text { for all } \xi \in W^{1, p} \\
\|\eta\|_{0, q}<\infty
\end{array}\right.
$$

where $\langle$,$\rangle is the standard Hermitian inner product on \mathbb{C}^{n}$. Since $\eta$ is smooth by the elliptic regularity, we integrate by parts to get

$$
\operatorname{Re} \int_{\Theta}\langle\bar{\partial} \xi, \eta\rangle=-\operatorname{Re} \int_{\Theta}\langle\xi, \partial \eta\rangle+\operatorname{Re} \int_{\partial \Theta}\langle\xi, \eta\rangle i d z
$$

where $z=x+i y$ is the standard coordinates of $\Theta$ considered as a subset in $\mathbb{C}$. Using the fact that

$$
\xi\left(\ell_{i}\right) \subset T \tilde{\Lambda}=\mathbb{R}^{n},
$$

we derive the equation from (6.30) and (6.31),

$$
\left\{\begin{array}{l}
\partial \eta=0 \\
\left.\eta\right|_{\ell_{i}} \subset T \tilde{\Lambda}_{i}=\mathbb{R}^{n} \\
\|\eta\|_{0, q}<\infty
\end{array}\right.
$$

By the same way as in the case of Ker $D \bar{\partial}_{\widetilde{w}}$, we conclude Coker $D_{\widetilde{w}_{0}} \bar{\partial}=\{0\}$. This finishes the proof.

Lemma 6.2 implies that there exists the inverse

$$
Q_{0}: L^{p}\left(\Omega^{(0,1)}\left(\widetilde{w}_{0}^{*} T \mathbb{C}^{n}\right)\right) \rightarrow W^{1, p}\left(\widetilde{w}_{0}^{*} T \mathbb{C}^{n}\right)
$$

of $D_{\widetilde{w}_{0}} \bar{\partial}$ such that

$$
D_{\widetilde{w}_{0}} \bar{\partial} \circ Q_{0}=\mathrm{id}, Q_{0} \circ D_{\widetilde{w}_{0}} \bar{\partial}=\mathrm{id} \text { and }\left\|Q_{0}\right\| \leq C_{9} .
$$

Using this, we are ready to describe the portion of $\xi$ on $\Theta_{0}\left(\frac{1}{\epsilon_{\alpha}}\right)$. Given $\eta \in \widetilde{\mathcal{H}}_{w^{\epsilon}}^{p}$, we first define

$$
\widetilde{\eta}(z):= \begin{cases}D \exp _{x}^{-1}\left(w^{\epsilon}(z)\right) \eta(z) & \text { on } \Theta_{0}\left(\frac{3}{2 \epsilon^{\alpha}}\right) \\ 0 & \text { otherwise. }\end{cases}
$$


Then $\widetilde{\eta}$ is a section of the bundle

$$
\Omega^{(0,1)}\left(\left(\epsilon \widetilde{w}^{\epsilon}(z)\right)^{*} T \mathbb{C}^{n}\right) \text { not } \Omega^{(0,1)}\left(\left(\epsilon \widetilde{w}_{0}\right)^{*} T \mathbb{C}^{n}\right)
$$

where

$$
\widetilde{w}^{\epsilon}(z)=\frac{1}{\epsilon} \exp _{x}^{-1}\left(w^{\epsilon}(z)\right)
$$

However, we have shown in Remark 4.3 that

$$
\widetilde{w}^{\epsilon}(z) \rightarrow \widetilde{w}_{0} \quad \text { as } \quad \epsilon \rightarrow 0 \text { in the } C^{1}-\text { topology. }
$$

Therefore, we will just pretend $\tilde{\eta}$ is an element in $\Omega^{(0,1)}\left(\left(\epsilon \widetilde{w}_{0}\right)^{*} T \mathbb{C}^{n}\right)$.

Now, we define the portion of $\xi$ on $\Theta_{0}\left(\frac{1}{\epsilon^{\alpha}}\right)$ by

$$
\xi(z)=D \exp _{x}\left(\epsilon \widetilde{w}_{0}\right)\left(Q_{0}^{\epsilon}(\widetilde{\eta})\left(\epsilon \widetilde{w}_{0}(z)\right)\right)
$$

where

$$
Q_{0}^{\epsilon}: L_{\epsilon}^{p}\left(\Omega^{(0,1)}\left(\left(\epsilon \widetilde{w}_{0}\right)^{*} T \mathbb{C}^{n}\right)\right) \rightarrow W_{\epsilon}^{1, p}\left(\left(\epsilon \widetilde{w}_{0}\right)^{*} T \mathbb{C}^{n}\right)
$$

is the operator obtained from

$$
Q_{0}: L^{p}\left(\Omega^{(0,1)}\left(\widetilde{w}_{0}\right)^{*} T \mathbb{C}^{n}\right) \rightarrow W^{1, p}\left(\left(\widetilde{w}_{0}\right)^{*} T \mathbb{C}^{n}\right)
$$

by

$$
Q_{0}^{\epsilon}(\zeta)(z)=Q_{0}^{\epsilon}(\zeta)\left(\epsilon \widetilde{w}_{0}(z)\right):=Q_{0}(\zeta \circ \epsilon)\left(\widetilde{w}_{0}(z)\right)
$$

where $\zeta \circ \epsilon$ is the element in $\Omega^{(0,1)}\left(\widetilde{w}_{0}^{*} T \mathbb{C}^{n}\right)$ defined by

$$
\zeta \circ \epsilon(z)=\zeta \circ \epsilon\left(\widetilde{w}_{0}(z)\right):=\zeta\left(\epsilon \widetilde{w}_{0}(z)\right) .
$$

One can easily check that the norms of $Q_{0}^{\epsilon}$ and $Q_{0}$ are the same.

Again we note that $\xi$ does not quite satisfy the right boundary conditions

$$
\xi\left(\ell_{i}\right) \subset T \Lambda_{i}^{\epsilon} \quad i=1,2,3
$$

but we ignore this in the same reason as before.

Finally, we recall from Remark 4.3 that $\exp \epsilon \widetilde{w}_{0}(z)$ and $\phi_{\epsilon t}^{f_{i+1}} \phi_{\epsilon(1-t)}^{f_{i}}\left(\chi_{i}(\epsilon \tau)\right)$ are $C^{1}$-close to each other as $\epsilon \rightarrow 0$ on $\Theta\left(\frac{1}{\epsilon^{\alpha}}, \frac{2}{\epsilon^{\alpha}}\right)$. Therefore if we denote by $\Pi_{\infty}(z)$ and $\Pi_{\infty}(z)$ the parallel translations along the shortest geodesics from

$$
\phi_{\epsilon t}^{f_{i+1}} \circ \phi_{\epsilon(1-t)}^{f_{i}}\left(\chi_{i}(\epsilon \tau)\right)\left(=\exp _{x} \epsilon \widetilde{w}_{i}^{\epsilon}(z)\right)
$$

and

$$
\exp _{x} \epsilon\left(\widetilde{w}_{0}(z)\right)
$$

to

$$
\exp _{x} \epsilon\left(\widetilde{w}_{0}(z)+\beta\left(\epsilon^{\alpha} \tau\right)\left(\widetilde{w}_{i} \epsilon(z)-\widetilde{w}_{0}(z)\right)\right.
$$

respectively, it follows that

$$
\left\|\Pi_{0}-\mathrm{id}\right\|_{C^{1}} \quad \text { or } \quad\left\|\Pi_{\infty}-\mathrm{id}\right\|_{C^{1}} \leq C \epsilon^{1-\alpha}
$$

Now, we define the operator

$$
Q_{\epsilon}: \widetilde{\mathcal{H}}_{\epsilon}^{p} \rightarrow W_{\epsilon}^{1, p}\left(\left(w^{\epsilon}\right)^{*} T X\right)
$$


by

$$
\xi:=Q_{\epsilon}(\eta)= \begin{cases}T\left(\phi_{t}^{\epsilon f_{i+1}} \circ \phi_{(1-t)}^{\epsilon f_{i}}\right) a_{i}^{\epsilon}(\tau, t)=: \xi_{\chi_{i}^{\epsilon}} & \text { for } \tau \leq \frac{2}{\epsilon^{\alpha}} \\ D \exp _{x}\left(\epsilon \widetilde{w}_{0}\right)\left(\widetilde{Q}_{0}(\widetilde{\eta})\left(\widetilde{w}_{0}(z)\right)\right. & \text { for } z \in \Theta_{0}\left(\frac{1}{\epsilon^{\alpha}}\right) \\ \beta\left(\epsilon^{\alpha} \tau\right) \Pi_{\infty}(z)\left(\xi_{\chi_{i}^{\epsilon}}\right)+\left(1-\beta\left(\epsilon^{\alpha} \tau\right)\right) \Pi_{0}(z)( & D \exp _{x}\left(\epsilon \widetilde{w}_{0}\right)\left(Q_{0}(\widetilde{\eta})\right) \\ & \text { for } z \in \Theta_{0}\left(\frac{1}{\epsilon^{\alpha}}, \frac{2}{\epsilon^{\alpha}}\right) .\end{cases}
$$

For the simplicity of exposition, we have denoted

$$
\xi_{\chi_{i}^{\epsilon}}=T\left(\phi_{t}^{\epsilon f_{i+1}} \circ \phi_{(1-t)}^{\epsilon f_{i}}\right) a_{i}^{\epsilon}(\tau, t) .
$$

7. Estimates of the inverse. The main result in this section will be the following.

Proposition 7.1. There exists $\epsilon_{3}>0$ such that if $0<\epsilon<\epsilon_{3}$, the operator $Q_{\epsilon}: \widetilde{\mathcal{H}}_{\epsilon}^{p} \rightarrow W_{\epsilon}^{1, p}\left(\left(w^{\epsilon}\right)^{*} T X\right)$ defined as in (6.34) satisfies the estimates (6.2).

Proof. The boundedness of $Q_{\epsilon}$ is easy to see from definition and so its proof will be omitted. Let $\left\|Q_{\epsilon}\right\| \leq C_{10}$. Therefore to prove the proposition we should prove that for any $\eta \in L_{\epsilon}^{p}\left(\Lambda^{(0,1)} T^{*} \Theta \otimes_{J}\left(w^{\epsilon}\right)^{*} T X\right)$, we have

$$
\left\|D_{w^{\epsilon}} \xi-\eta\right\|_{0, p, \epsilon} \leq \frac{1}{2}\|\eta\|_{0, p, \epsilon}
$$

where $\xi=Q_{\epsilon}(\eta)$ is defined as in (6.31).

We will estimate norms $\|\cdot\|_{0, p, \epsilon}$ separately in each region considered before. have

We start with the region $\Theta_{i}\left(\frac{2}{\epsilon^{\alpha}}\right)$. In terms of the coordinates $(\tau, t)$ on $\Theta_{i}\left(\frac{2}{\epsilon^{\alpha}}\right)$, we

$$
\begin{aligned}
D_{w^{\epsilon}} \xi & =D \bar{\partial}_{J}\left(w^{\epsilon}\right) \cdot \xi \\
& =\left(\nabla_{\tau}+J \nabla_{t}\right) \xi+\nabla_{t} J \cdot \xi .
\end{aligned}
$$

By the definition of $w^{\epsilon}$ in (4.9), of $a_{i}^{\epsilon}$ in (6.3) and (6.25) and of $\xi$ in (6.34), it is easy to see that

$$
\xi(\tau, t)=\left.\frac{d}{d \delta}\right|_{\delta=0} \phi_{t}^{\epsilon f_{i+1}} \circ \phi_{(1-t)}^{\epsilon f_{i}}\left(\lambda_{i, \delta}^{\epsilon}\right)
$$

where $\lambda_{i, \delta}^{\epsilon}:(-\infty, 0] \times[0,1] \rightarrow X$ for $\delta \in(-\epsilon, \epsilon)$ is a family of maps such that

$$
\lambda_{i, 0}^{\epsilon}(\tau, t)=\chi_{i}(\epsilon \tau)=\chi_{i}^{\epsilon}(\tau) \quad \text { and }\left.\quad \frac{d}{d \delta}\right|_{\delta=0} \lambda_{i, \delta}^{\epsilon} \equiv a_{i}^{\epsilon}
$$

on $\Theta_{i}\left(\frac{2}{\epsilon^{\alpha}}\right)$. Therefore, a straightforward computation, using the properties of the Levi-Civita connection, (7.1) and Lemma 3.4, gives rise to the identities

$$
\nabla_{\tau} \xi=T\left(\phi_{t}^{\epsilon f_{i+1}} \circ \phi_{(1-t)}^{\epsilon f_{i}}\right) \nabla_{\tau} a_{i}^{\epsilon}
$$

and

$$
\left.\nabla_{t} \xi=T\left(\phi_{t}^{\epsilon f_{i+1}} \circ \phi_{(1-t)}^{\epsilon f_{i}}\right)\left\{\epsilon \nabla X_{\left(F_{i+1}-F_{i}\right)}\right)\left(a_{i}^{\epsilon}\right)+\nabla_{t} a_{i}^{\epsilon}\right\}
$$

Hence

$$
\left(\nabla_{\tau}+J \nabla_{t}\right) \xi=T\left(\phi_{t}^{\epsilon f_{i+1}} \circ \phi_{(1-t)}^{\epsilon f_{i}}\right)\left\{\nabla_{\tau} a_{i}^{\epsilon}+\left(\phi_{t}^{\epsilon f_{i+1}} \circ \phi_{(1-t)}^{\epsilon f_{i}}\right)^{*} J\left(\nabla_{t} a_{i}^{\epsilon}+\epsilon \nabla X_{\left(F_{i+1}-F_{i}\right)} a_{i}^{\epsilon}\right)\right\} .
$$


Substituting (6.25) into this, we get

$$
\left.\left(\nabla_{\tau}+J \nabla_{t}\right) \xi=T\left(\phi_{t}^{\epsilon f_{i+1}} \circ \phi_{(1-t)}^{\epsilon f_{i}}\right)\left\{\left(\phi_{t}^{\epsilon f_{i+1}} \circ \phi_{(1-t)}^{\epsilon f_{i}}\right)^{*} J-J\right)\left(\nabla_{t} a_{i}^{\epsilon}+\epsilon \nabla X_{\left(F_{i+1}-F_{i}\right)} a_{i}^{\epsilon}\right)+b_{i}^{\epsilon}\right\}
$$

on $\Theta_{i}\left(\frac{2}{\epsilon^{\alpha}}\right)$. Recalling the definition (6.3) of $b_{i}^{\epsilon}$, we have on $\Theta_{i}\left(\frac{2}{\epsilon^{\alpha}}\right)$

$$
\eta=T\left(\phi_{t}^{\epsilon f_{i+1}} \circ \phi_{(1-t)}^{\epsilon f_{i}}\right) b_{i}^{\epsilon}
$$

and so

$$
\begin{aligned}
D_{w^{\epsilon}} \xi(\tau, t)-\eta(\tau, t)= & T\left(\phi_{t}^{\epsilon f_{i+1}} \circ \phi_{(1-t)}^{\epsilon f_{i}}\right)\left\{\left(\phi_{t}^{\epsilon f_{i+1}} \circ \phi_{(1-t)}^{\epsilon f_{i}}\right)^{*} J-J\right) \times \\
& \left.\left(\nabla_{t} a_{i}^{\epsilon}+\epsilon \nabla X_{\left(F_{i+1}-F_{i}\right.} a_{i}^{\epsilon}\right)\right\}+\nabla_{t} J \xi(\tau, t)
\end{aligned}
$$

The first term in (7.5) has norm bounded by

$$
\left.C_{11} \epsilon\left(\left|\nabla_{t} a_{i}^{\epsilon}\right|+\epsilon \mid \nabla X_{\left(F_{i+1}-F_{i}\right)}\right) a_{i}^{\epsilon} \mid\right) \text {. }
$$

By summing the $L_{\epsilon}^{p}$-norm over $i=1,2,3$, we obtain from (6.4) and (6.25)

$$
\begin{aligned}
\sum_{i=1}^{3} C_{11} \epsilon & \left\|\left|\nabla_{t} a_{i}^{\epsilon}\right|+\epsilon\left|\nabla X_{\left(F_{i+1}-F_{i}\right)} a_{i}^{\epsilon}\right|\right\|_{0, p, \epsilon, \Theta_{i}\left(\frac{2}{\epsilon^{\alpha}}\right)} \\
& \leq C_{12} \epsilon\left\|\vec{a}^{\epsilon}\right\|_{1, p, \epsilon} \\
& \leq C_{8} C_{12} \epsilon\left\|\vec{b}^{\epsilon}\right\|_{1, p, \epsilon} .
\end{aligned}
$$

For the second term $\left|\nabla_{t} J \cdot \xi(\tau, t)\right|$ in (7.5), we note that

$$
\left|\nabla_{t} J\right|=\left|\nabla_{t}\left(J\left(w^{\epsilon}(\tau, t)\right)\right)\right| \leq C \epsilon
$$

and so $\nabla_{t} J \cdot \xi(\tau, t)$ has the norm as a one form

$$
\begin{aligned}
\sum_{i=1}^{3} \| \nabla_{t} J & \cdot \xi \|_{0, p, \epsilon, \Theta_{i}\left(\frac{2}{\epsilon^{\alpha}}\right)}^{p}=\sum_{i=1}^{3} \int_{\Theta_{i}\left(\frac{2}{\epsilon^{\alpha}}\right)} \epsilon^{2-p}\left|\nabla_{t} J \cdot \xi(\tau, t)\right|^{p} \\
& \leq C^{p} \epsilon^{p} \sum_{i=1}^{3} \int_{\Theta_{i}\left(\frac{2}{\epsilon^{\alpha}}\right)} \epsilon^{2-p}\left|a_{i}^{\epsilon}(\tau, t)\right|^{p} \leq C^{p} \epsilon^{p}\|\vec{a}\|_{1, p, \epsilon}^{p} \\
& \leq C^{p} \epsilon^{p} C_{8}^{p}\left\|\vec{b}^{\epsilon}\right\|_{0, p, \epsilon}^{p} \\
& \leq C_{13}^{p} \epsilon^{p}\|\eta\|_{0, p, \epsilon}^{p}
\end{aligned}
$$

and hence,

$$
\sum_{i=1}^{3}\left\|\nabla_{t} J \cdot \xi\right\|_{0, p, \epsilon, \Theta_{i}\left(\frac{2}{\epsilon^{\alpha}}\right)}^{p} \leq C_{13}^{p} \epsilon^{p}\|\eta\|_{0, p, \epsilon}^{p} .
$$

Combining (7.5), (7.6) and (7.7), we have obtained

$$
\left\|D_{w^{\epsilon}} \xi-\eta\right\|_{0, p, \epsilon, \Theta \backslash \Theta_{0}\left(\frac{2}{\epsilon^{\alpha}}\right)}^{p} \leq\left(C_{8}^{p} C_{12}^{p} \epsilon^{p}+C_{13}^{p} \epsilon^{p}\right)\|\eta\|_{0, p, \epsilon}^{p} \leq C_{14}^{p} \epsilon^{p}\|\eta\|_{0, p, \epsilon}^{p} .
$$

Next, we estimate

$$
\left\|D_{w^{\epsilon}} \xi-\eta\right\|_{0, p, \epsilon, \Theta_{0}\left(\frac{1}{\epsilon^{\alpha}}\right)}
$$

Since $\xi=D \exp _{x}\left(\epsilon \widetilde{w}_{0}\right) Q_{0}(\widetilde{\eta})$ on $\Theta_{0}\left(\frac{1}{\epsilon^{\alpha}}\right)$, we have

$$
D_{w^{\epsilon}} \xi=D_{w^{\epsilon}}\left(D \exp _{x}\left(\epsilon \tilde{w}_{0}\right) Q_{0}(\tilde{\eta})\right) .
$$


We give the standard coordinates $(x, y)$ on $\Theta_{0}\left(\frac{1}{\epsilon^{\alpha}}\right)$ as a subset of $\mathbb{C}$ and compute

$$
\begin{aligned}
D_{w^{\epsilon}} \xi= & \left(\nabla_{x}+J \nabla_{y}+\nabla_{y} J\right) \xi \\
= & \left(\nabla_{x}+J \nabla_{y}+\nabla_{y} J\right)\left(D \exp _{x}\left(\epsilon \widetilde{w}_{0}\right) Q_{0}(\widetilde{\eta})\right) \\
= & \left(\nabla_{x}+J \nabla_{y}\right)\left(D \exp _{x}\left(\epsilon \widetilde{w}_{0}\right)\right) Q_{0}(\widetilde{\eta}) \\
& +D \exp _{x}\left(\epsilon \widetilde{w}_{0}\right)\left(\nabla_{x}+\left(\exp _{x}\left(\epsilon \widetilde{w}_{0}\right)\right)^{*} J \nabla_{y}\right) Q_{0}(\widetilde{\eta}) \\
& +\nabla_{y} J \cdot D \exp _{x}\left(\epsilon \widetilde{w}_{0}\right) Q_{0}(\widetilde{\eta}) .
\end{aligned}
$$

First note that we have

$$
\begin{aligned}
\left|\nabla\left(D \exp _{x}\left(\epsilon \widetilde{w}_{0}\right)\right) \cdot Q_{0}(\widetilde{\eta})\right| & \leq\left|\nabla\left(D \exp _{x}\left(\epsilon \widetilde{w}_{0}\right)\right)\right|\left|Q_{0}(\widetilde{\eta})\right| \\
& \leq C \epsilon^{1-\alpha}\left|Q_{0}(\widetilde{\eta})\right|
\end{aligned}
$$

on $\Theta\left(\frac{1}{\epsilon^{\alpha}}\right)$, where the second inequality follows from the inequality

$$
\left|\epsilon \widetilde{w}_{0}\right| \leq C \epsilon^{1-\alpha}
$$

and from the standard property of the exponential map. Using (7.10), the first term of (7.9) can be estimated as

$$
\begin{aligned}
\|\left(\nabla_{x}\right. & \left.\left.+J \nabla_{y}\right) D \exp _{x}\left(\epsilon \widetilde{w}_{0}\right)\right) Q_{0}(\widetilde{\eta}) \|_{0, p, \epsilon, \Theta_{0}\left(\frac{1}{\epsilon^{\alpha}}\right)}^{p} \\
& =\int_{\Theta_{0}\left(\frac{1}{\epsilon^{\alpha}}\right)} \epsilon^{2-p}\left|\left(\nabla_{x}+J \nabla_{y}\right)\left(D \exp _{x}\left(\epsilon \widetilde{w}_{0}\right)\right) Q_{0}(\widetilde{\eta})\right|^{p} \\
& =\int_{\Theta_{0}\left(\frac{1}{\epsilon^{\alpha}}\right)} \epsilon^{2-p} C^{p} \epsilon^{p-p \alpha}\left|Q_{0}(\widetilde{\eta})\right|^{p} \\
& =C^{p} \epsilon^{2-p \alpha} \int_{\Theta_{0}\left(\frac{1}{\epsilon^{\alpha}}\right)}\left|Q_{0}(\widetilde{\eta})\right| p \\
& \leq C^{p} \epsilon^{2-p \alpha}\left\|Q_{0}(\widetilde{\eta})\right\|_{0, p}^{p} \leq C_{15}^{p} \epsilon^{2-p \alpha}\|\widetilde{\eta}\|_{0, p}^{p}
\end{aligned}
$$

On the other hand, we have

$$
\begin{aligned}
\|\widetilde{\eta}\|_{0, p}^{p} & =\int_{\Theta}|\widetilde{\eta}(z)|^{p} d z \\
& =\int_{\Theta_{0}\left(\frac{3}{2 \epsilon^{\alpha}}\right)}\left|D \exp _{x}^{-1}\left(w^{\epsilon}(z)\right) \eta(z)\right|^{p} d s \quad \text { from (6.32) } \\
& \leq C_{16}^{p} \int_{\Theta_{0}\left(\frac{3}{2 \epsilon^{\alpha}}\right)}|\eta(z)|^{p} d z
\end{aligned}
$$

By substituting this into (7.11), we obtain

$$
\begin{aligned}
\|\left(\nabla_{x}\right. & \left.+J \nabla_{y}\right)\left(D \exp _{x}\left(\epsilon \widetilde{w}_{0}\right)\right) Q_{0}(\widetilde{\eta}) \|_{0, p, \epsilon, \Theta_{0}\left(\frac{1}{\epsilon^{\alpha}}\right)}^{p} \\
\leq & C_{15}^{p} C_{16}^{p} \epsilon^{p-p \alpha} \int_{\Theta_{0}\left(\frac{3}{2 \epsilon^{\alpha}}\right)} \epsilon^{2-p}|\eta(z)|^{p} d z .
\end{aligned}
$$

and hence,

$$
\left\|\left(\nabla_{x}+J \nabla_{y}\right)\left(D \exp _{x}\left(\epsilon \widetilde{w}_{0}\right)\right) Q_{0}(\widetilde{\eta})\right\|_{0, p, \epsilon, \Theta_{0}\left(\frac{2}{\epsilon^{\alpha}}\right)} \leq C_{15} C_{16} \epsilon^{1-\alpha}\|\eta\|_{0, p, \epsilon}
$$

For the third term in (7.9), we immediately get

$$
\left\|\nabla_{y} J \cdot D \exp _{x}\left(\epsilon \widetilde{w}_{0}\right) Q_{0}(\widetilde{\eta})\right\|_{0, p, \epsilon, \Theta\left(\frac{1}{\epsilon^{\alpha}}\right)} \leq C_{17} \epsilon^{1-\alpha}\|\eta\|_{0, p, \epsilon, \Theta\left(\frac{1}{\epsilon^{\alpha}}\right)}
$$


For the second term in (7.9), we rewrite

$$
\begin{aligned}
& \left.D \exp _{x}\left(\epsilon \widetilde{w}_{0}\right)\left(\nabla_{x}+\left(\exp _{x}\left(\epsilon \widetilde{w}_{0}\right)\right)^{*} J \nabla_{y}\right) Q_{0}(\widetilde{\eta})\right) \\
& =D \exp _{x}\left(\epsilon \widetilde{w}_{0}\right)\left(\nabla_{x}+J_{0} \nabla_{y}\right) Q_{0}(\widetilde{\eta}) \\
& \quad+D \exp _{x}\left(\epsilon \widetilde{w}_{0}\right)\left(\left(D \exp _{x}\left(\epsilon \widetilde{w}_{0}\right)\right)^{*} J-J_{0}\right) Q_{0}(\widetilde{\eta}) \\
& \quad \quad \text { where } \quad J_{0}=J(x) \\
& =D \exp _{x}\left(\epsilon \widetilde{w}_{0}\right) D_{\widetilde{w}_{0}} \circ Q_{0}(\widetilde{\eta})+D \exp _{x}\left(\epsilon \widetilde{w}_{0}\right)\left(\left(D \exp _{x}\left(\epsilon \widetilde{w}_{0}\right)\right)^{*} J-J_{0}\right) Q_{0}(\widetilde{\eta})
\end{aligned}
$$

By the same way as before, the second term here can be estimated

$$
\| D \exp _{x}\left(\epsilon \widetilde{w}_{0}\right)\left(\left(D \exp _{x}\left(\epsilon \widetilde{w}_{0}\right)^{*} J-J_{0}\right) Q_{0}(\widetilde{\eta})\left\|_{0, p, \epsilon, \Theta_{0}\left(\frac{1}{\epsilon^{\alpha}}\right)} \leq C_{18} \epsilon^{1-\alpha}\right\| \eta \|\right.
$$

On the other hand, we have

$$
\begin{aligned}
D \exp _{x} & \left(\epsilon \widetilde{w}_{0}\right)\left(D_{\widetilde{w}_{0}} \circ Q_{0}(\widetilde{\eta})\right)=D \exp _{x}\left(\epsilon \widetilde{w}_{0}\right)(\widetilde{\eta}) \quad \text { since } D_{\widetilde{w}_{0}} \circ Q_{0}=\text { id } \\
& =D \exp _{x}\left(\epsilon \widetilde{w}_{0}\right) D \exp _{x}^{-1}\left(w^{\epsilon}\right) \eta \\
& =\eta+\left(D \exp _{x}\left(\epsilon \widetilde{w}_{0}\right) D \exp _{x}^{-1}\left(w^{\epsilon}\right)-\mathrm{id}\right) \cdot \eta
\end{aligned}
$$

Hence, combining (7.10), (7.12), (7.13), (7.14) and (7.15), we have obtained

$$
\left\|D_{w^{\epsilon}} \xi-\eta\right\|_{0, p, \epsilon, \Theta_{0}\left(\frac{1}{\epsilon^{\alpha}}\right)}^{p} \leq C_{19} \epsilon^{(1-\alpha) p}\|\eta\|_{0, p . \epsilon}^{p}
$$

Finally we need to estimate $D_{w^{\epsilon}} \xi-\eta$ on the intermediate regions $\Theta_{i}\left(\frac{1}{\epsilon^{\alpha}}, \frac{2}{\epsilon^{\alpha}}\right)$. We recall

$$
\xi=Q_{\epsilon}(\eta)=\beta\left(\epsilon^{\alpha} \tau\right) \Pi_{\infty}(z)\left(\xi_{\chi_{i}}(z)\right)+\left(1-\beta\left(\epsilon^{\alpha} \tau\right)\right) \Pi_{0}(z)\left(D \exp _{x}(\epsilon \widetilde{w})\left(Q_{0}(\widetilde{\eta})\right)\right)
$$

for $z \in \Theta_{i}\left(\frac{1}{\epsilon^{\alpha}}, \frac{2}{\epsilon^{\alpha}}\right)$. Therefore,

$$
\begin{aligned}
D_{w^{\epsilon}} \xi- & \eta=D_{w^{\epsilon}}\left\{\beta\left(\epsilon^{\alpha} \tau\right) \Pi_{\infty}(z)\left(\xi_{\chi_{i}}(z)\right)\right\} \\
& +D_{w^{\epsilon}}\left\{\left(1-\beta\left(\epsilon^{\alpha} \tau\right)\right) \Pi_{0}(z)\left(D \exp _{x}\left(\epsilon \widetilde{w}_{0}\right)\left(Q_{0}(\widetilde{\eta})\right)\right)\right\}-\eta \\
= & \epsilon^{\alpha} \beta^{\prime}\left(\epsilon^{\alpha} \tau\right)\left(\Pi_{\infty}(z) \xi_{\chi_{i}}(z)-\Pi_{0}(z)\left(D \exp _{x}\left(\epsilon \widetilde{w}_{0}\right)\left(Q_{0}(\widetilde{\eta})\right)\right)\right. \\
& +\beta\left(\epsilon^{\alpha} \tau\right) D_{w^{\epsilon}}\left(\Pi_{\infty}(z)\left(\xi_{\chi_{i}}(z)\right)\right)+ \\
& +\left(1-\beta\left(\epsilon^{\alpha} \tau\right)\right) D_{w^{\epsilon}}\left(\Pi_{0}(z)\left(D \exp _{x}\left(\epsilon \widetilde{w}_{0}\right)\left(Q_{0}(\widetilde{\eta})\right)\right)\right)-\eta
\end{aligned}
$$

Here, the first term can be easily estimated as before to get

$$
\begin{gathered}
\| \epsilon^{\alpha} \beta^{\prime}\left(\epsilon^{\alpha} z\right)\left(\Pi_{\infty}(z) \xi_{\chi_{i}}-\Pi_{0}(z)\left(D \exp _{x}\left(\epsilon \widetilde{\omega}_{0}\right)\left(Q_{0}(\widetilde{\eta})\right) \|_{0, p, \epsilon, \Theta_{0}\left(\frac{1}{\epsilon^{\alpha}}, \frac{2}{\epsilon^{\alpha}}\right)}^{p}\right.\right. \\
\leq C_{20}^{p} \epsilon^{\alpha p}\|\eta\|_{0, p, \Theta}^{p}
\end{gathered}
$$

To estimate the second term, we consider the regions

$$
\Theta_{i}\left(\frac{1}{\epsilon^{\alpha}}, \frac{3}{2 \epsilon^{\alpha}}\right) \text { and } \Theta_{i}\left(\frac{3}{2 \epsilon^{\alpha}}, \frac{2}{\epsilon^{\alpha}}\right)
$$

separately. First, consider the region $\Theta_{i}\left(\frac{1}{\epsilon^{\alpha}}, \frac{3}{2 \epsilon^{\alpha}}\right)$. In this region, we recall that

$$
b_{i}^{\epsilon} \equiv 0
$$


and so

$$
\widetilde{D}_{\chi_{i}^{\epsilon}} a_{i}^{\epsilon} \equiv 0
$$

Now, using the fact

$$
\left\|D_{w^{\epsilon}} \cdot \Pi_{\infty}(z)-\Pi_{\infty}(z) D_{w_{i}^{\epsilon}}\right\|_{C^{1}} \leq C_{21} \epsilon^{1-\alpha}
$$

and

$$
\left\|D_{w^{\epsilon}} \cdot \Pi_{0}(z)-\Pi_{0}(z) D_{w_{i}^{\epsilon}}\right\|_{C^{1}} \leq C_{21} \epsilon^{1-\alpha}
$$

we have

$$
\begin{aligned}
& \| \beta\left(\epsilon^{\alpha} \tau\right) D_{w^{\epsilon}}\left(\Pi_{\infty}(z)\left(\xi_{\chi_{i}}(z)\right)+\right. \\
& \quad+\left(1-\beta\left(\epsilon^{\alpha} \tau\right)\right) D_{w^{\epsilon}}\left(\Pi_{0}(z)\left(D \exp _{x}\left(\epsilon \widetilde{w}_{0}\right) Q_{0}(\widetilde{\eta})\right)-\eta \|_{0, p, \Theta_{i}\left(\frac{1}{\epsilon^{\alpha}}, \frac{3}{2 \epsilon^{\alpha}}\right)}^{p}\right. \\
& \leq\left\|\beta\left(\epsilon^{\alpha} \tau\right) \Pi_{\infty}(z) D_{w_{i}^{\epsilon}} \xi_{x_{i}}+\left(1-\beta\left(\epsilon^{\alpha} \tau\right)\right) \Pi_{0}(z) D_{w_{0}^{\epsilon}}\left(D \exp _{x}\left(\epsilon \widetilde{w}_{0}\right) Q_{0}(\widetilde{\eta})\right)-\eta\right\|_{0, p, \Theta_{i}\left(\frac{1}{\epsilon}\right)}^{p} \\
& \quad+C_{21} \epsilon^{p(1-\alpha)}\|\eta\|_{0, p, \Theta_{i}\left(\frac{1}{\epsilon^{\alpha}}, \frac{3}{2 \epsilon^{\alpha}}\right)}^{p}
\end{aligned}
$$

On the other hand, by writing

$$
\begin{aligned}
& \beta\left(\epsilon^{\alpha} \tau\right) \Pi_{\infty}(z) D_{w_{i}^{\epsilon}} \xi_{\chi_{i}}+\left(1-\beta\left(\epsilon^{\alpha} \tau\right)\right) \Pi_{0}(z) D_{w_{0}^{\epsilon}}\left(D \exp _{x}\left(\epsilon \widetilde{w}_{0}\right) Q_{0}(\widetilde{\eta})\right)-\eta \\
& =\beta\left(\epsilon^{\alpha} \tau\right)\left(\Pi_{\infty}(z) D_{w_{i}^{\epsilon}} \xi_{\chi_{i}}-\eta\right)+ \\
& \quad+\left(1-\beta\left(\epsilon^{\alpha} \tau\right)\right)\left(\Pi_{0}(z) D_{w_{0}^{\epsilon}}\left(D \exp _{x}\left(\epsilon \widetilde{w}_{0}\right) Q_{0}(\widetilde{\eta})\right)-\eta\right)
\end{aligned}
$$

and then using the estimates similar to (7.8) and (7.16) to each term above, we can obtain

$$
\begin{aligned}
\| \beta\left(\epsilon^{\alpha} \tau\right) \Pi_{\infty}(z) D_{w_{i}^{\epsilon}} \xi_{\chi_{i}} & +\left(1-\beta\left(\epsilon^{\alpha} \tau\right)\right) \Pi_{0}(z) D_{w_{0}^{\epsilon}}\left(D \exp _{x}\left(\epsilon \tilde{w}_{0}\right) Q_{0}(\widetilde{\eta})\right)-\eta \|_{0, p, \Theta\left(\frac{1}{\epsilon^{\alpha}}, \frac{3}{2 \epsilon^{\alpha}}\right)}^{p} \\
& \leq C_{22}^{p} \epsilon^{p(1-\alpha)}\|\eta\|_{0, p, \Theta}^{p} .
\end{aligned}
$$

Similar estimates can be carried out for the region $\Theta_{i}\left(\frac{3}{2 \epsilon^{\alpha}}, \frac{2}{\epsilon^{\alpha}}\right)$. From this together with (7.17) and (7.18), we have obtained

$$
\left\|D_{w^{\epsilon}} \xi-\eta\right\|_{0, p, \Theta_{i}\left(\frac{1}{\epsilon^{\alpha}}, \frac{2}{\epsilon^{\alpha}}\right)}^{p} \leq\left(C_{20}^{p} \epsilon^{\alpha p}+C_{22}^{p} \epsilon^{p(1-\alpha)}\right)\|\eta\|_{0, p, \Theta}^{p}
$$

Finally by adding (7.8), (7.16), (7.17) and (7.18), we have obtained the estimate

$$
\left\|D_{w^{\epsilon}} \xi-\eta\right\|_{0, p, \Theta}^{p} \leq\left(C_{14}^{p} \epsilon^{p}+C_{19}^{p} \epsilon^{(1-\alpha) p}+C_{20}^{p} \epsilon^{\alpha p}+C_{22}^{p} \epsilon^{p(1-\alpha)}\right)\|\eta\|_{0, p, \Theta}^{p}
$$

and so for sufficiently small $\epsilon>0$, we have proven

$$
\left\|D_{w^{\epsilon}} \xi-\eta\right\|_{0, p, \Theta} \leq \frac{1}{2}\|\eta\|_{0, p, \Theta}
$$

which finally finishes the proof of (6.2) and hence Proposition 7.1.

8. Proof of Theorem 3.1. Using the estimates we have established in the previous sections, we are now ready to construct the map

$$
\Phi^{\epsilon}: \mathcal{M}(M: \vec{f}, \vec{p}) \rightarrow \mathcal{M}_{J}\left(X: \vec{\Lambda}^{\epsilon}, \vec{x}^{\epsilon}\right) .
$$


We will do this in two steps. First, we note the map defined in (4.9) that defines approximate $J$-holomorphic maps is obviously smooth map from $\mathcal{M}(M: \vec{f}, \vec{p})$ into $\mathcal{F}_{\epsilon}^{1, p}$. We denote this map by $\Phi_{1}^{\epsilon}: \mathcal{M} \rightarrow \mathcal{F}_{\epsilon}^{1, p}$ which is defined by

$$
\Phi_{1}^{\epsilon}(I):=w^{\epsilon, I}
$$

Now, we would like to apply Proposition 3.2 and 3.3 to $w=w^{\epsilon, I}$ (with $\xi=0$ ) in Proposition 3.2 in the second step. In Proposition 5.3, we have proven the estimate

$$
\left\|\bar{\partial}_{J} w^{\epsilon, I}\right\|_{0, p, \epsilon} \leq C_{6} \epsilon^{\frac{2+(p-1) \alpha}{p}}
$$

for all $I \in \mathcal{M}(M: \vec{f}, \vec{p})$ and in Proposition 7.1 we have obtained the estimate for the approximate right inverse

$$
\left\|Q_{\epsilon}\right\| \leq C_{7}, \quad\left\|D_{w^{\epsilon}} \circ Q_{\epsilon}-\mathrm{id}\right\|<\frac{1}{2}
$$

which will in turn imply the estimate

$$
\left\|Q_{w^{\epsilon}}\right\| \leq C_{23}
$$

where $Q_{w^{\epsilon}}=Q_{\epsilon}\left(D_{w^{\epsilon}} \circ Q_{\epsilon}\right)^{-1}$ is a right inverse of $D_{w^{\epsilon}}$. The estimate

$$
\left\|D w^{\epsilon, I}\right\| \leq C_{0}
$$

is obvious. These estimates (8.2), (8.3) and (8.4) with $\xi=0$ satisfy all the requirements for us to apply Proposition 3.2 to solve the following equation

$$
\bar{\partial}_{J}\left(\exp _{w^{\epsilon, I}} Q_{w^{\epsilon, I}} \eta\right)=0
$$

in terms of $\eta$. In other words, we have proven that there exist some $\epsilon_{4}>0$ such that for $0<\epsilon<\epsilon_{4}$. there exists $\eta=\eta(\epsilon, I) \in \widetilde{H}_{w^{\epsilon, I}}^{p}$ which solves (8.5) and which depends smoothly on $\epsilon$ and $I \in \mathcal{M}(M: \vec{f}, \vec{p})$. The smooth dependence follows from the content of the implicit function theorem and the uniqueness statement in Proposition 3.3. Finally, our required map

$$
\Phi^{\epsilon}: \mathcal{M}(M: \vec{f}, \vec{p}) \rightarrow \mathcal{M}_{J}\left(T^{*} M: \vec{\Lambda}^{\epsilon}, \vec{x}^{\epsilon}\right)
$$

is defined by

$$
\begin{aligned}
\Phi^{\epsilon}(I) & =\exp _{w^{\epsilon, I}}\left(Q_{w^{\epsilon, I}} \eta(\epsilon, I)\right) \\
& =\exp _{\Phi_{1}^{\epsilon}(I)}\left(Q_{\Phi_{1}^{\epsilon}(I)} \eta(\epsilon, I)\right) .
\end{aligned}
$$

To finish the proof that this map $\Phi^{\epsilon}$ is indeed a smooth proper diffeomorphism, we proceed in four steps:

STEP I. $\Phi^{\epsilon}$ is a local diffeomorphism,

STEP II. $\Phi^{\epsilon}$ is a surjective map,

STEP III. $\Phi^{\epsilon}$ is a proper map.

By combining these three steps, we conclude that $\Phi^{\epsilon}$ is a finite covering map.

STEP IV. $\Phi^{\epsilon}$ is a one to one map.

8.1. Step I (Local diffeomorphism). To prove $\Phi^{\epsilon}$ is a local diffeomorphism, it will be enough to prove that the derivative

$$
T \Phi^{\epsilon}(I): T_{I} \mathcal{M}(M: \vec{f}, \vec{p}) \rightarrow T_{\Phi^{\epsilon}(I)} \mathcal{M}_{J}\left(T^{*} M: \vec{\Lambda}^{\epsilon}, \vec{x}^{\epsilon}\right)
$$


is an isomorphism for all $I$. First it follows from the index computation of the linearized operator

$$
D \bar{\partial}_{J}\left(\Phi^{\epsilon}(I)\right): T_{\Phi^{\epsilon}(I)} \mathcal{F}_{\epsilon}^{1, p} \rightarrow \widetilde{\mathcal{H}}_{\Phi^{\epsilon}(I)}^{p}
$$

and

$$
L_{I}: W_{I}^{1, p} \rightarrow L_{\chi_{1}}^{p} \times L_{\chi_{2}}^{p} \times L_{\chi_{3}}^{p}
$$

that both have the same Fredholm indices (See [Fu2]). Furthermore by making a generic choice of $\vec{f}$ that satisfies the transversality condition imposed as in Theorem 3.1, we may assume that both operators are surjective by Proposition 6.1. We also note that

$$
\begin{gathered}
T_{I} \mathcal{M}(M: \vec{f}, \vec{p}) \cong \operatorname{Ker} L_{I} \\
T_{\Phi^{\epsilon}(I)} \mathcal{M}_{J}\left(T^{*} M: \vec{\Lambda}^{\epsilon}, \vec{x}^{\epsilon}\right) \cong \operatorname{Ker} D \bar{\partial}_{J}\left(\Phi^{\epsilon}(I)\right) .
\end{gathered}
$$

Now using the fact that the map

$$
T \Phi_{1}^{\epsilon}(I): T_{I} \mathcal{M}(M: \vec{f}, \vec{p}) \rightarrow T_{\Phi_{1}^{\epsilon}(I)} \Phi_{1}(\mathcal{M})
$$

is an isomorphism which can be easily checked from definition of $\Phi_{1}^{\epsilon}(I)=w^{\epsilon, I}$ and the fact that that map $T \Phi^{\epsilon}(I): T_{I} \mathcal{M} \rightarrow T_{\Phi^{\epsilon}(I)} \mathcal{M}_{J}$ factors through by the diagram.

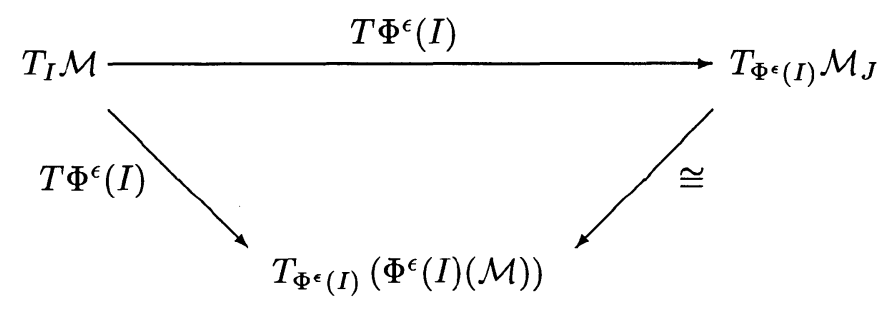

FIGURE 8.1

Here the isomorphism on the right hand side arrow comes from the content of the implicit function theorem Proposition 3.2.

8.2. Step II: Surjectiveness. Since this proof will be quite involved and long, we will postpone the proof to the next section. This is the step where we have to use the canonical complex structure $J_{g}$ on $T^{*} M$ that is induced from the metric $g$ on $M$.

8.3. Step III: Properness. By definition of $\Phi_{1}^{\epsilon}$ in (8.1) and in Section 4, the properness of $\Phi_{1}^{\epsilon}$ is obvious. Since we have the estimates

$$
\|\xi\|_{1, p, \epsilon}=\left\|Q_{\Phi_{1}^{\epsilon}} \eta(\epsilon, I)\right\| \leq C \epsilon^{\frac{2+(p-1) \alpha}{p}}
$$

from Proposition 3.2 and (8.2), we obtain

$$
\|\xi\|_{1, p} \leq C \epsilon^{\frac{(p-1) \alpha}{p}} .
$$

from the relation between the ordinary and the weighted Sobolev norms

$$
\|\xi\|_{1, p} \leq C \epsilon^{-\frac{2}{p}}\|\xi\|_{1, p, \epsilon}
$$

Then the (ordinary) Sobolev inequality implies

$$
\operatorname{dist}\left(\Phi_{1}^{\epsilon}(I)(\tau, t), \Phi^{\epsilon}(I)(\tau, t)\right) \leq C \epsilon^{\frac{(p-1) \alpha}{p}}
$$


for all $I \in \mathcal{M}(M: \vec{f}, \vec{p})$. Now we give the proof of the properness of $\Phi^{\epsilon}$. Suppose that $\Phi^{\epsilon}$ is not proper and then there exist a sequence $\left\{I_{k}\right\} \subset \mathcal{M}(M: \vec{f}, \vec{p})$ with $V\left(I_{k}\right) \rightarrow \infty$ (i.e, diverges) but $\Phi^{\epsilon}\left(I_{k}\right)$ converges. By choosing a subsequence if necessary, we may assume that for all $k$

$$
\lim _{\tau \rightarrow-\infty} \Phi^{\epsilon}\left(\left.I_{k}\right|_{e_{i}}\right)=x_{i}^{\epsilon}
$$

for $i=1,2,3$ respectively. We choose $\epsilon$ so small and fixed that

$$
C \epsilon^{\frac{(p-1) \alpha}{p}}<\frac{1}{2} D^{0}
$$

where

$$
D^{0}=\min _{p, p^{\prime}} d\left(p, p^{\prime}\right) \quad \text { where } \quad p, p^{\prime} \in \cup_{i=1}^{3}\left(\operatorname{Crit}\left(f_{i+1}-f_{i}\right)\right)
$$

which is independent of $\epsilon$. Note that if $\epsilon$ is sufficiently small, it is easy to see from the identity $x_{i}^{\epsilon}=\left(p_{i}, \epsilon d f_{i}\right)$ that

$$
D^{\epsilon} \geq \frac{1}{2} D^{0}
$$

where

$$
D^{\epsilon}:=\min _{x, x^{\prime}} d\left(x, x^{\prime}\right) \quad \text { where } \quad x, x^{\prime} \in \cup_{i=1}^{3}\left(\Lambda_{i+1}^{\epsilon} \cap \Lambda_{i}^{\epsilon}\right) .
$$

We choose $\epsilon_{5}>0$ such that if $0<\epsilon<\epsilon_{5}$, then all the above inequalities hold. We fix any such $\epsilon>0$. Since $\Phi_{1}^{\epsilon}$ is proper and $I_{k}$ diverges as $k \rightarrow \infty, \Phi_{1}^{\epsilon}\left(I_{k}\right)$ diverges and so by the weak convergence theorem, there exists a sequence $\tau_{k} \rightarrow \infty$ and some $i$ among $i=1,2,3$ such that as $k \rightarrow \infty$

$$
\Phi_{1}^{\epsilon}\left(\left.I_{k}\right|_{e_{i}}\right)\left(\tau_{k}, \cdot\right) \rightarrow \tilde{x} \text { where } x_{i}^{\epsilon} \neq \tilde{x} \in \cup_{i=1}^{3}\left(L_{i+1}^{\epsilon} \cap L_{i}^{\epsilon}\right) .
$$

However since $\Phi^{\epsilon}\left(I_{k}\right)$ converges, we have as $k \rightarrow \infty$

$$
\Phi^{\epsilon}\left(\left.I_{k}\right|_{e_{i}}\right)\left(\tau_{k}, \cdot\right) \rightarrow x_{i}^{\epsilon} .
$$

Combining (8.9)-(8.13), we get a contradiction which finishes the proof of the properness of $\Phi^{\epsilon}$ for any $0<\epsilon<\epsilon_{5}$.

Combining Step I, II and III, we have proven that

$$
\Phi^{\epsilon}: \mathcal{M}_{g}(M: \vec{f}, \vec{p}) \rightarrow \mathcal{M}_{J}\left(T^{*} M: \vec{\Lambda}^{\epsilon}, \vec{x}^{\epsilon}\right)
$$

is a covering projection with finite sheets.

8.4. Step IV: Injectivity. Suppose the contrary, i.e., these exist $I \neq I^{\prime}$ such that

$$
\Phi^{\epsilon}(I)=\Phi^{\epsilon}\left(I^{\prime}\right)
$$

Since $\Phi^{\epsilon}$ is a covering map, we must have

$$
\left\|I-I^{\prime}\right\| \geq \delta^{\prime}>0
$$

for some $\delta^{\prime}$ for all $0<\epsilon<\epsilon_{6}$ which depends only on $\delta$ in Proposition 3.2. This follows from the uniform invertibility of $D \Phi^{\epsilon}(I): T_{I} \mathcal{M} \rightarrow T_{\Phi^{\epsilon}(I)} \mathcal{M}_{J}$ (see Proposition 7.1). Hence from the definition of $\Phi_{1}^{\epsilon}$, it is easy to check that

$$
\left\|\Phi_{1}^{\epsilon}(I)-\Phi_{1}^{\epsilon}\left(I^{\prime}\right)\right\|_{L^{\infty}} \geq \frac{1}{2} \delta^{\prime}>0
$$


by choosing smaller $\epsilon$ if necessary. Now (8.14) and (8.15) contradict to each other by the estimates (8.7), provided $\epsilon$ is sufficiently small. This finishes the proof of the injectivity of $\Phi^{\epsilon}$.

Finally, it remains to prove the surjectivity of the map $\Phi^{\epsilon}$ which we will do in the next section.

9. Surjectivity of the map $\Phi^{\epsilon}$. We first note that from the uniqueness statement in Proposition 3.3 together with the estimate (8.10), the surjectivity of the map $\Phi^{\epsilon}$ will follow from the following theorem.

Theorem 9.1. Let $J=J_{g}$ be the canonical almost complex structure on $X$ as in (1.4). And let $\delta>0$ be the constant given in Proposition 3.3. Then there exists $\epsilon_{7}>0$ such that if $0<\epsilon<\epsilon_{7}$, for each given $w \in \mathcal{M}_{J}\left(X: \vec{\Lambda}^{\epsilon}, \vec{x}^{\epsilon}\right)$ there exists some

$$
I=I(w) \in \mathcal{M}_{g}(M: \vec{f}, \vec{p}) \quad \text { and } \eta \in \tilde{\mathcal{H}}_{\Phi_{1}^{\epsilon}(I)}^{\epsilon}
$$

such that

$$
w=\exp _{\Phi_{1}^{\epsilon}(I)}\left(Q_{\Phi_{1}^{\epsilon}(I)} \eta\right)
$$

with

$$
\left\|Q_{\Phi_{1}^{\epsilon}(I)} \eta\right\|_{L^{\infty}} \leq \frac{\delta}{2}
$$

The following two lemmas are the first step to the proof of the theorem.

Lemma 9.2. Let $J$ be any almost complex structure compatible with $\omega$ on $T^{*} M$. Then there exists a constant $C_{24}=C_{24}(\vec{f})$ depending only on $\vec{f}=\left(f_{1}, f_{2}, f_{3}\right)$ such that

$$
\int u^{*} \omega=\int_{\Theta}|D w|_{J}^{2} \leq C_{24} \epsilon
$$

for all $w \in \mathcal{M}_{J}\left(X: \vec{\Lambda}^{\epsilon}, \vec{x}^{\epsilon}\right)$, where the norm $|\cdot|_{J}$ on $T^{*} M$ is the one induced from the metric $g(\cdot, \cdot)=\omega(\cdot, J \cdot)$.

Proof. Since $w$ is $J$-holomorphic and $J$ is compatible to the standard complex structure $\omega$ on $X=T^{*} M$, we have the following well-known identity

$$
\frac{1}{2} \int_{\Theta}|D w|_{J}^{2}=\int_{\Theta} w^{*} \omega
$$

Since $w=-d \theta, \theta$ is the canonical one form on $T^{*} M$, we have from the Stoke's formula

$$
\int w^{*} \omega=-\int_{\Theta} w^{*} d \theta=-\int_{\partial \Theta} w^{*} \theta=\sum_{j=1}^{3}\left(-\int_{\ell_{j}} w^{*} \theta\right) .
$$

On the other hand, since $\Lambda_{j}^{\epsilon}=$ Graph $\epsilon d f_{j}$ and $w\left(\ell_{j}\right) \subset \Lambda_{j}^{\epsilon}$, we have

$$
-\int_{\ell_{j}} w^{*} \theta=-\int_{w\left(\ell_{j}\right)} \epsilon d f_{j}=-\epsilon\left(f_{j}\left(p_{j+1}\right)-f_{j}\left(p_{j}\right)\right)
$$

and by summing up, we have obtained

$$
\frac{1}{2} \int_{\Theta}|D w|_{J}^{2}=\sum_{j=1}^{3}\left(-\int_{\ell_{j}} w^{*} \theta\right)=\epsilon \sum_{j=1}^{3}\left(f_{j}\left(p_{j+1}\right)-f_{j}\left(p_{j}\right)\right)
$$

and hence

$$
\frac{1}{2} \int_{\Theta}|D w|_{J}^{2} \leq 3 \epsilon \sum_{j=1}^{3}\left(\max f_{j}-\min f_{j}\right)
$$


By setting $C_{24}=3 \sum_{j=1}^{3}\left(\max f_{j}-\min f_{j}\right)$, we are done.

Lemma 9.3 [Corollary 3.4, Remark after Corollary 3.5; 01]. Denote by $D$ either the open unit disc or the half-open disc with boundary $\partial D=(-1,1)$. Let $u: D \rightarrow(P, \omega, J)$ be a map such that

$$
u(\partial D) \subset L \text { and } \quad \bar{\partial}_{J} u=0
$$

where $L$ is a given compact Lagrangian submanifold of $(P, \omega)$. We denote by

$$
\begin{gathered}
\epsilon_{8}=\inf \left\{\int u^{*} \omega \mid u:\left(D^{2}, \partial D^{2}\right) \rightarrow(P, L) \text { or } u: S^{2} \rightarrow P\right. \\
\text { and } \left.\bar{\partial}_{J} u=0 \quad \text { and nonconstant }\right\}
\end{gathered}
$$

where $D^{2}$ is the unit disc. Then for any $r<1$ and $u$ with $\int_{D}|D u|^{2}<\epsilon_{8}$, we have

$$
\max _{|x|<r}|D u(x)| \leq C_{25}(r)\|D u\|_{2, D(1)}=C_{25}(r)\left(\int_{D(1)}|D u|^{2}\right)^{\frac{1}{2}} \text {. }
$$

We remark that in the present case where $P=T^{*} M$ and $L=$ Graph $d g$ for a function $g$ on $M$, we have $\epsilon_{8}=\infty$ in (9.3) because there exists no nontrivial $J$ holomorphic sphere or disc with boundary on $L$. Hence the uniform estimates (9.3) hold for all (local) $J$-holomorphic map $u:(D, \partial D) \rightarrow\left(T^{*} M, L\right)$ where $D$ as in Lemma 9.3. Combining (9.2) and (9.3) together with the exponential decay of $w \in \mathcal{M}_{J}\left(T^{*} M\right.$ : $\left.\vec{\Lambda}^{\epsilon}, \vec{x}^{\epsilon}\right)$, we have obtained

$$
\sup _{z \in \Theta}|D w(z)| \leq C_{26} \sqrt{\epsilon}
$$

for all $w \in \mathcal{M}_{J}\left(T^{*} M: \vec{\Lambda}^{\epsilon}, \vec{x}^{\epsilon}\right)$ where $C_{26}=C_{26}(\vec{f})$ depends only on $\vec{f}$. From the boundary condition $w\left(\ell_{i}\right) \subset \Lambda_{i}^{\epsilon}$ and from the fact that $\Lambda_{i}^{\epsilon} \rightarrow M$ as $\epsilon \rightarrow 0$ in $T^{*} M$, (9.4) immediately implies

$$
\sup _{z \in \Theta} d(w(z), M) \leq C_{26} \sqrt{\epsilon}
$$

However, the estimate (9.4) is not strong enough to analyze the degeneration of $\mathcal{M}_{J}\left(T^{*} M: \vec{\Lambda}^{\epsilon}, \vec{x}^{\epsilon}\right)$ to $\mathcal{M}_{g}(M: \vec{f}, \vec{p})$ and we need to improve (9.4) to the estimate

$$
\sup _{z \in \Theta}|D w(z)| \leq C \epsilon .
$$

This is one of the reason why we restrict to the canonical almost complex structure $J=J_{g}$ induced from the Riemannian metric $g$ on $M$. The other reason was in the proof of the transversality result in terms of the gradient flows which was carried out in Section 6.

Proposition 9.4. Let $g$ be a Riemannian metric on $M$ and $J=J_{g}$ be the canonical almost complex structure on $T^{*} M$ induced from the Levi-Civita connection of $g$. Then there exists $\epsilon_{8}>0$ and $C_{27}>0$ such that if $0<\epsilon<\epsilon_{8}$ and $w \in \mathcal{M}_{J}\left(T^{*} M\right.$ : $\left.\vec{\Lambda}^{\epsilon}, \vec{x}^{\epsilon}\right)$, we have

$$
\sup _{z \in \Theta} d(w(z), M) \leq C_{27} \epsilon
$$

Remark 9.5. In fact, the above $C^{0}$-estimate can be proven for any almost complex structure $J$ compatible to $\omega$, if we allow to vary the metric $g$ on $M$ appropriately in terms of the almost complex structure $J$ on $T^{*} M$. This variation will be necessary, when one attempts to prove the result as in this paper (in the presence of bubbling) 
for the case of more general Lagrangian submanifolds in the general symplectic manifold $(P, \omega)$. This proof uses a different argument using the fact that the cotangent bundle is "convex" in that the level hypersurfaces of the radial function are (pseudo)convex in the sense of symplectic geometry. But we prefer to use the above more standard method in this paper, where it is enough to consider the case in which $J$ is the canonical structure associated to the fixed metric $g$.

Proof. The Levi-Civita connection of $g$ induces the splitting

$$
T_{\xi}\left(T^{*} M\right)=H_{\xi} \oplus V_{\xi}, \quad \xi \in T^{*} M
$$

into the horizontal and vertical subspaces. In particular when $\xi \in M \subset T^{*} M$, this splitting coincides with the canonical splitting

$$
T_{\xi}\left(T^{*} M\right) \cong T_{\pi(\xi)} M \oplus T_{\pi(\xi)}^{*} M
$$

and so $J_{g}$ maps the vector $v \in T M$ into the co-vector $g(v, \cdot)$. Furthermore it is well-known that there exists the canonical identification of $V_{\xi}$ with $T_{\pi(\xi)}^{*} M$ where $\pi: T^{*} M \rightarrow M$ is the projection. We denote by

$$
\Pi_{H}, \Pi_{V}: T\left(T^{*} M\right) \rightarrow T\left(T^{*} M\right)
$$

the horizontal and vertical projections with respect to the splitting (9.7). Now we choose the standard coordinates $z=x+i y$ on $\Theta$ as a subset of $\mathbb{C}$ and identify $\bar{\partial}_{J} w$ as a section of $w^{*} T M$ as before. By decomposing $\bar{\partial}_{J} w$ into horizontal and vertical components, we can rewrite $\bar{\partial}_{J} w=0$ into

$$
\left\{\begin{array}{l}
\Pi_{H}\left(\frac{\partial w}{\partial x}+J \frac{\partial w}{\partial y}\right)=\Pi_{H}\left(\frac{\partial w}{\partial x}\right)+J \Pi_{V}\left(\frac{\partial w}{\partial y}\right)=0 \\
\Pi_{V}\left(\frac{\partial w}{\partial x}+J \frac{\partial w}{\partial y}\right)=\Pi_{V}\left(\frac{\partial w}{\partial x}\right)+J \Pi_{H}\left(\frac{\partial w}{\partial y}\right)=0
\end{array}\right.
$$

by the definition of $J=J_{g}$. We write $w(z)=(q(z), p(z))$ for $p(z) \in T_{q(z)}^{*} M$. Then after we apply $T \pi: T\left(T^{*} M\right) \rightarrow T M$ to it, (9.8) becomes

$$
\frac{\partial q}{\partial x}+T \pi \circ J \nabla_{y} p=0
$$

and after we apply $T \pi \circ J: T\left(T^{*} M\right) \rightarrow T M,(9.9)$ becomes

$$
\frac{\partial q}{\partial y}-T \pi \circ J \nabla_{x} p=0
$$

Therefore the equation $\bar{\partial}_{J} w=0$ becomes

$$
\left\{\begin{array}{r}
\frac{\partial q}{\partial x}+T \pi \circ J \nabla_{y} p=0 \\
\frac{\partial q}{\partial y}-T \pi \circ J \nabla_{x} p=0
\end{array}\right.
$$

We would like to emphasize that $\nabla p \cong \Pi_{V} \circ D w$ is the covariant derivative of $p$ considered as a section of $T^{*} M$ along $q$ and $T \pi \circ J(w)$ is a section of the bundle $w^{*} \operatorname{End}\left(T^{*} M, T M\right)$. In particular when Image $w \subset M \subset T^{*} M, T \pi \circ J(w)$ becomes the natural map

$$
g(v, \cdot) \mapsto-v
$$

To prove (9.6), it is enough to prove

$$
|p(z)| \leq C \epsilon
$$


for all $z \in \theta$ and $w \in \mathcal{M}_{J}\left(T^{*} M: \vec{\Lambda}^{\epsilon}, \vec{x}^{\epsilon}\right)$. Due to the boundary condition

$$
w\left(\ell_{i}\right) \subset \Lambda_{i}^{\epsilon}=\text { Graph } \epsilon d f_{i},
$$

this holds on $\partial \Theta$. Therefore this will immediately follow from the following lemma.

Lemma 9.6. There exists $\epsilon_{9}>0$ depending only on $\vec{f}=\left(f_{1}, f_{2}, f_{3}\right)$ such that if $0<\epsilon<\epsilon_{9}$, the function

$$
z \rightarrow|p(z)|^{2}, \quad z \in \Theta
$$

is a subharmonic function and so any local maximum of the function is attained on $\partial \Theta$.

Proof. We compute $\Delta\langle p, p\rangle$ where $\Delta=\frac{\partial^{2}}{\partial x^{2}}+\frac{\partial^{2}}{\partial y^{2}}$ :

$$
\frac{\partial^{2}}{\partial x^{2}}\langle p, p\rangle+\frac{\partial^{2}}{\partial y^{2}}\langle p, p\rangle \text {. }
$$

And

$$
\begin{aligned}
& \frac{\partial^{2}}{\partial x^{2}}\langle p, p\rangle=2\left\langle\nabla_{x} p, \nabla_{x} p\right\rangle+2\left\langle\nabla_{x} \nabla_{x} p, p\right\rangle \\
& \frac{\partial^{2}}{\partial y^{2}}\langle p, p\rangle=2\left\langle\nabla_{y} p, \nabla_{y} p\right\rangle+2\left\langle\nabla_{y} \nabla_{y} p, p\right\rangle .
\end{aligned}
$$

Using (9.10) and (9.11), we compute

$$
\begin{aligned}
\nabla_{x} \nabla_{x} p & =\nabla_{x}\left((T \pi \circ J)^{-1} \frac{\partial q}{\partial y}\right) \\
& =\left(\nabla_{x}(T \pi \circ J)^{-1}\right) \frac{\partial q}{\partial y}+(T \pi \circ J)^{-1} \nabla_{x}\left(\frac{\partial q}{\partial y}\right)
\end{aligned}
$$

and

$$
\begin{aligned}
\nabla_{y} \nabla_{y} p & =-\nabla_{y}\left((T \pi \circ J)^{-1} \frac{\partial q}{\partial x}\right) \\
& =-\left(\nabla_{y}(T \pi \circ J)^{-1}\right) \frac{\partial q}{\partial x}-(T \pi \circ J)^{-1} \nabla_{y}\left(\frac{\partial q}{\partial y}\right)
\end{aligned}
$$

Since $\nabla_{x}\left(\frac{\partial q}{\partial y}\right)=\nabla_{y}\left(\frac{\partial q}{\partial x}\right)$ by the symmetry of the Levi-Civita connection, adding (9.12) and (9.13), we have

$$
\begin{aligned}
\Delta\langle p, p\rangle= & 2\left(\left\langle\nabla_{x} p, \nabla_{x} p\right\rangle+\left\langle\nabla_{y} p, \nabla_{y} p\right\rangle\right) \\
& +\left\langle\left(\nabla_{x}(T \pi \circ J)^{-1}\right) \frac{\partial q}{\partial y}-\left(\nabla_{y}(T \pi \circ J)^{-1}\right) \frac{\partial q}{\partial x}, p\right\rangle
\end{aligned}
$$

Again from (9.10), (9.11), the second term in (9.14) becomes

$$
\left\langle\left(\nabla_{x}(T \pi \circ J)^{-1}\right)(T \pi \circ J) \nabla_{x} p+\left(\nabla_{y}(T \pi \circ J)^{-1}\right)(T \pi \circ J) \nabla_{y} p, p\right\rangle:=A .
$$

Using the crude estimates (9.4) or (9.5) and the equation (9.10), (9.11), it is easy to see

$$
\left|\nabla(T \pi \circ J)^{-1}\right| \leq C\left(\left|\nabla_{x} p\right|+\left|\nabla_{y} p\right|\right)
$$

and so we have

$$
\begin{aligned}
|A| & \leq C_{28}\left(\left|\nabla_{y} p\right|+\left|\nabla_{x} p\right|\right)^{2}|p| \\
& \leq C_{28} C_{26} \sqrt{\epsilon}\left(\left|\nabla_{y} p\right|+\left|\nabla_{x} y\right|\right)^{2} \\
& \leq C_{29} \sqrt{\epsilon}\left(\left|\nabla_{y} p\right|^{2}+\left|\nabla_{x} p\right|^{2}\right) .
\end{aligned}
$$


Substituting this into (9.14), we have obtained

$$
\begin{aligned}
\Delta\langle p, p\rangle & \geq\left(2-C_{29} \sqrt{\epsilon}\right)\left(\left|\nabla_{x} p\right|^{2}+\left|\nabla_{y} p\right|^{2}\right) \\
& =\left(2-C_{29} \sqrt{\epsilon}\right)|\nabla p|^{2} \geq|\nabla p|^{2} \geq 0
\end{aligned}
$$

provided $\epsilon$ is sufficiently small. This finishes the proof of Lemma 9.6 and so Proposition 9.4 .

With the $C^{0}$-estimate, we now proceed the $C^{1}$-estimate.

Proposition 9.7. There exists $\epsilon_{9}>0$ and $C_{30}>0$ such that

$$
\sup _{z \in \Theta}|D w(z)| \leq C_{30} \epsilon
$$

for all $w \in \mathcal{M}_{J}\left(X: \vec{\Lambda}^{\epsilon}, \vec{x}^{\epsilon}\right)$ provided $0<\epsilon<\epsilon_{9}$.

Proof. Once we have the $C^{0}$-estimate in Proposition 9.4, the proof of (9.15) is just a standard blowing-up argument. We will therefore sketch the essential part of details. Suppose (9.15) does not hold. Then there exists a sequence $\epsilon_{k} \rightarrow 0$ and $w_{k} \in \mathcal{M}_{J}\left(X: \vec{\Lambda}^{\epsilon_{k}}, \vec{x}^{\epsilon_{k}}\right)$ such that there exists $z_{k} \in \Theta$ with

$$
\begin{aligned}
& \left|D w_{k}\left(z_{k}\right)\right|=\max _{z \in \Theta}|D w(z)| \\
& R_{k}:=\frac{1}{\epsilon_{k}}\left|D w_{k}\left(z_{k}\right)\right| \rightarrow \infty
\end{aligned}
$$

as $k \rightarrow \infty$. Since $M$ is compact and from (9.6), we may assume, by choosing a subsequence if necessary, that

$$
w\left(z_{k}\right) \rightarrow q \in M \subset X .
$$

Using the exponential map $\exp _{q}: T_{q} X \rightarrow X$, we can write

$$
\begin{aligned}
& w_{k}\left(z_{k}+u\right)=\exp _{q} \xi_{k}(u) \text { or } \\
& \xi_{k}(u)=\exp _{q}^{-1}\left(w_{k}\left(z_{k}+u\right)\right.
\end{aligned}
$$

for some map

$$
\xi_{k}: \Theta_{z_{k}} \subset \mathbb{C} \rightarrow T_{q} X
$$

which is defined on

$$
\Theta_{z_{k}}:=\left\{u \in \mathbb{C}|| u \mid \leq 1, z_{k}+u \in \Theta \subset \mathbb{C}\right\}
$$

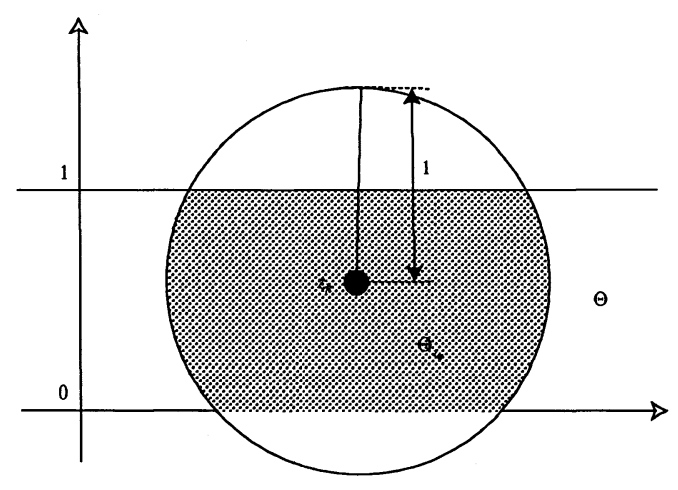


Now we define maps $\widetilde{w}_{k}$ into $T_{q} X$ by

$$
\widetilde{w}_{k}(v)=\frac{1}{\epsilon} \xi_{k}\left(\frac{v}{R_{k}}\right)=\frac{1}{\epsilon} \exp _{q}^{-1}\left(w_{k}\left(z_{k}+\frac{v}{R_{k}}\right)\right)
$$

for $v \in \mathbb{C}$ with $\frac{v}{R_{k}} \in \Theta_{z_{k}}$. The domain of $\widetilde{w}_{k}$ is

$$
\widetilde{\Theta}_{k}=\left\{v \in \mathbb{C}|| v \mid \leq R_{k} \text { and } z_{k}+\frac{v}{R_{k}} \in \Theta\right\} .
$$

Furthermore $\widetilde{w}_{k}$ have the properties that

$$
\left|\operatorname{Im} \widetilde{w}_{k}\right| \text { is bounded }
$$

by (9.6) and as $\epsilon \rightarrow 0$,

$$
\begin{aligned}
& \left|D \widetilde{w}_{k}(z)\right| \leq 1+o(1) \quad \text { for all } z \in \widetilde{\Theta}_{k} \\
& \left|D \widetilde{w}_{k}(0)\right|=1 .
\end{aligned}
$$

Now depending on whether

$$
\begin{aligned}
& \frac{1}{\epsilon_{k}} \operatorname{dist}\left(z_{k}, \partial \Theta\right) \rightarrow \infty \quad \text { or } \\
& \frac{1}{\epsilon_{k}} \operatorname{dist}\left(z_{k}, \partial \Theta\right) \rightarrow C<\infty,
\end{aligned}
$$

we will have obtained a non-trivial $J_{0}$-holomorphic map into $\left(T_{q} X, J_{0}\right) \cong \mathbb{C}^{n}$ as a $C^{1}$-limit of $\widetilde{w}_{k}$, which will be defined either on $\mathbb{C}$ or on the (conformally) upper halfspace $\mathbb{C}_{+}$and whose imaginary part is uniformly bounded. Then by the maximum principle, this limit must be a constant. On the other hand, by the $C^{1}$-convergence and by (9.19), this gives rise to a contradiction. Hence the proof.

Now we are ready to prove Theorem 9.1 and the rest of the section will be spent to prove it. We start with the center region $\Theta_{0}\left(\frac{2}{\epsilon^{\alpha}}\right)$. We apply the estimates $(9.16)$ to each point in $\Theta_{0}\left(\frac{2}{\epsilon^{\alpha}}\right)$ and then we get

$$
\max _{z \in \Theta_{0}\left(\frac{2}{\epsilon^{\alpha}}\right)} d(w(0), w(z)) \leq C_{31} \epsilon^{1-\alpha} .
$$

Since we assume $0<\alpha<1$, we have

$$
\lim _{\epsilon \rightarrow 0} \max _{z \in \Theta_{0}\left(\frac{2}{\epsilon^{\alpha}}\right)} d(w(0), w(z))=0 .
$$

Now we consider the regions $\Theta_{i}\left(\frac{2}{\epsilon^{\alpha}}\right), i=1,2,3$. For given $w \in \mathcal{M}_{J}\left(X: \vec{\Lambda}^{\epsilon}, \vec{x}^{\epsilon}\right)$, we apply the reverse construction of (4.9). In other words, on $\Theta_{j}\left(\frac{2}{\epsilon^{\alpha}}\right)$ we define

$$
\tilde{\chi}_{w}(\sigma, s)=\left(\phi_{s}^{f_{i+1}} \circ \phi_{\epsilon-s}^{f_{i}}\right)^{-1} w\left(\frac{\sigma+i s}{\epsilon}\right)
$$

or

$$
w\left(\frac{\sigma+i s}{\epsilon}\right)=\left(\phi_{s}^{f_{j+1}} \circ \phi_{\epsilon-s}^{f_{j}}\right) \tilde{\chi}_{w}(\sigma, s)
$$

for $(\sigma, s)$ such that

$$
-\infty<\sigma \leq 2 \epsilon^{1-\alpha}, \quad 0 \leq s \leq \epsilon
$$

and so

$$
\left(\frac{\sigma}{s}, \frac{s}{\epsilon}\right) \in \Theta_{j}\left(\frac{2}{\epsilon^{\alpha}}\right) .
$$

A straightforward computation, using the equation $\bar{\partial}_{J} w=0$ and Lemma 3.4, we obtain

$$
\frac{\partial \widetilde{\chi}_{w}}{\partial \sigma}+\left(\phi_{s}^{f_{i+1}} \circ \phi_{\epsilon-s}^{f_{i}}\right)^{*} J\left(\frac{\partial \widetilde{\chi}_{w}}{\partial s}+X_{F_{i+1}-F_{i}}\right)=0 .
$$


For the sake of simplicity, we denote

$$
\begin{aligned}
J_{\epsilon}^{i}(s, x) & :=\left(\phi_{s}^{f_{i+1}} \circ \phi_{\epsilon-s}^{f_{i}}\right)^{*} J(x) \\
\lambda_{\epsilon}^{i}(s) & :=\left.\left(\phi_{s}^{f_{i+1}} \circ \phi_{\epsilon-s}^{f_{i}}\right)^{-1} w\right|_{\Theta_{i}}\left(0, \frac{s}{\epsilon}\right)
\end{aligned}
$$

for $s \in[0, \epsilon]$ and $x \in T^{*} M$. With these notations, $\tilde{\chi}_{w}$ satisfies the equation

$$
\left\{\begin{array}{l}
\frac{\partial \tilde{\chi}_{w}}{\partial \sigma}+J_{\epsilon}^{i}(s, x)\left(\frac{\partial \tilde{\chi}_{w}}{\partial s}+X_{\left(F_{i+1}-F_{i}\right)}\right)=0 \\
\tilde{\chi}_{w}(0, s)=\lambda_{\epsilon}^{i}(s) \\
\lim _{\sigma \rightarrow-\infty} \tilde{\chi}_{w}(\sigma, s)=\left(\phi_{s}^{f_{i+1}} \circ \phi_{\epsilon-s}^{f_{i}}\right)^{-1} x_{i}^{\epsilon} \text { on } \Theta_{i} \\
\tilde{\chi}_{w}(\sigma, 0), \tilde{\chi}_{w}(\sigma, \epsilon) \in M \subset X=T^{*} M .
\end{array}\right.
$$

On the other hand, we look at the equation

$$
\left\{\begin{array}{l}
\frac{\partial \chi}{\partial \sigma}+\operatorname{grad}\left(f_{i+1}-f_{i}\right)(\chi)=0 \\
\chi(0)=\left.w\right|_{\Theta_{i}}(0,0) \in M
\end{array}\right.
$$

which can be considered the (singular) limit equation of (9.23). To prove Theorem 9.1 , it will be enough to prove that there exists some $\epsilon_{10}>0$ such that for any $w \in \mathcal{M}_{J}^{\epsilon}, 0<\epsilon<\epsilon_{10}$, there exists some

$$
I=I_{w}=\left(\chi_{1}, \chi_{2}, \chi_{3}\right) \in \mathcal{M}_{g}(M: \vec{f}, \vec{p})
$$

such that for each $i=1,2,3$, we have

$$
\sup _{\sigma} d\left(\tilde{\chi}_{w, i}(\sigma, 0), \chi_{k}(\sigma)\right) \leq \frac{\delta}{3} .
$$

Proposition 9.8. There exists $\epsilon_{11}>0$ such that for any given $w \in \mathcal{M}_{J}^{\epsilon}, 0<$ $\epsilon<\epsilon_{11}$ and for each $i=1,2,3$, there exists a map $\chi_{w}:(-\infty, 0] \rightarrow M$ that satisfies

$$
\frac{d \chi}{d \sigma}+\operatorname{grad}\left(f_{i+1}-f_{i}\right)(\chi)=0
$$

and which also satisfies

$$
\sup _{\sigma \in(-\infty, 0]} d\left(\tilde{\chi}_{w}(\sigma, 0), \chi_{w}(\sigma)\right) \leq \frac{\delta}{3} .
$$

Assuming this proposition for the moment, we proceed the proof of Theorem 9.1. By (9.15) and (9.24), we can write

$$
\tilde{\chi}_{w}(\sigma, s)=\exp _{\chi_{w}(\sigma)} \tilde{\xi}(\sigma, s)
$$

for $-\infty<\sigma \leq 0,0 \leq s \leq \epsilon$ with $\|\widetilde{\xi}\|_{L^{\infty}} \leq \frac{\delta}{5}$, and so we can write

$$
w(\tau, t)=\exp _{\Phi_{i}^{\epsilon}\left(I_{w}\right)} \xi(\tau, t)
$$

for some $\xi$ with

$$
\|\xi\|_{L^{\infty}} \leq \frac{\delta}{4} \quad \text { and } I_{w}=\left(\chi_{1}, \chi_{2}, \chi_{3}\right) \in \mathcal{M}_{g}(M: \vec{f}, \vec{p})
$$

where $\chi_{i}$ are the gradient trajectories of $f_{i+1}-f_{i}$ that are obtained from above for each $i=1,2,3$. Now we have only to prove, by further perturbing $I_{w}$ to $\left.\widetilde{I}_{w} \in \mathcal{M}_{(} M: \vec{f}, \vec{p}\right)$ if necessary, that we can choose $\widetilde{\xi}$ of the form $Q_{\Phi_{1}^{\epsilon}\left(\widetilde{I}_{w}\right)} \widetilde{\eta}$ and so $w$ has the required form

$$
w=\exp _{\Phi_{1}^{\epsilon}\left(\widetilde{I}_{w}\right)} Q_{\Phi_{1}^{\epsilon}\left(\widetilde{I}_{w}\right)} \tilde{\eta}
$$


for some $\tilde{\eta} \in \tilde{\mathcal{H}}_{\epsilon}^{p}$. We introduce the map

$$
E(x, y)=\exp _{x}^{-1}(y)
$$

which is well-defined whenever $x, y \in X$ satisfy

$$
d(x, y)<\text { injectivity radus of } X .
$$

(See e.g., $[\mathrm{K}]$ for the basic properties of the map $E$.) Using the map $E$, we need to solve the equation

$$
\Pi_{\text {Ker } D_{\Phi_{1}^{\epsilon}(I)}}\left(E\left(\Phi_{1}^{\epsilon}(I), w\right)\right)=0
$$

in terms of $I$. Here we denote by $\Pi_{\text {Ker } D_{\Phi_{1}^{\epsilon}(I)}}$ the $L^{2}$-projection onto Ker $D_{\Phi_{1}^{\epsilon}(I)}$ with respect to the splitting

$$
T_{\Phi_{1}^{\epsilon}(I)} \mathcal{F}_{\epsilon}^{1, p}=\operatorname{Ker} D_{\Phi_{1}^{\epsilon}(I)} \oplus \operatorname{Image} Q_{\Phi_{1}^{\epsilon}(I)} .
$$

We consider the vector bundle

$$
\bigcup_{I \in \mathcal{M}} \operatorname{Ker} D_{\Phi_{1}^{\epsilon}(I)}
$$

over $\mathcal{M}=\mathcal{M}(M: \vec{f}, \vec{p})$ and define a section $\xi_{0}^{\epsilon}$ by

$$
\xi_{0}^{\epsilon}=\Pi_{\text {Ker } D_{\Phi_{1}^{\epsilon}(I)}}\left(E\left(\Phi_{1}^{\epsilon}(I), w\right)\right) .
$$

Solving (9.26) is equivalent to finding a zero of the section $\xi_{0}^{\epsilon}$. We note that from (9.24) and the accompanied estimates, we have

$$
\left\|\xi_{0}^{\epsilon}\left(I_{w}\right)\right\|_{L^{\infty}} \leq \frac{\delta}{3}
$$

and this in turn gives rise to the estimates

$$
\left\|\xi_{0}^{\epsilon}\left(I_{w}\right)\right\|_{1, p, \epsilon} \leq C \delta
$$

by the elliptic boot-strap because both $w$ is holomorphic and $\Phi_{1}^{\epsilon}\left(I_{w}\right)$ is "nearly holomorphic". Therefore to prove the existence of zeros of $\xi_{0}^{\epsilon}$ near $I_{w}$, we have only to prove that the covariant linearization at $I_{w}$

$$
D \xi_{0}^{\epsilon}\left(I_{w}\right): T_{I_{w}} \mathcal{M} \rightarrow \operatorname{Ker} D_{\Phi_{1}^{\epsilon}\left(I_{w}\right)}
$$

is uniformly invertible over $\epsilon>0$. This is because we can apply the existence scheme of this paper to this finite dimensional picture which is much easier. However we have the formula

$$
D \xi_{0}^{\epsilon}\left(I_{w}\right)(\delta I)=\Pi_{\operatorname{Ker} D_{\Phi_{1}^{\epsilon}\left(I_{w}\right)}}\left(D_{1} E\left(\Phi_{1}^{\epsilon}\left(I_{w}\right), w\right) \circ D \Phi_{1}^{\epsilon}(\delta I)\right)+O(\delta) .
$$

From this, (8.17) and from the fact that Image $D \Phi_{1}^{\epsilon}\left(I_{w}\right)$ is almost the same as Ker $D_{\Phi_{1}^{\epsilon}\left(I_{w}\right)}$, it is straightforward to conclude that $D \xi_{0}^{\epsilon}\left(I_{w}\right)$ is uniformly invertible over $\epsilon>0$, which finishes the proof of Theorem 9.1.

Finally, it remains to prove Proposition 9.8.

Proof of Proposition 9.8. From the $C^{1}$-estimate (9.16) and from the definition (9.22), it follows by a straightforward computation that

$$
\left|\frac{\partial \tilde{\chi}_{w}}{\partial s}\right| \leq C
$$


for all $w \in \mathcal{M}_{J}\left(X: \vec{\Lambda}^{\epsilon}, \vec{x}^{\epsilon}\right)$ and for all $\epsilon>0$. From (9.23) and (9.27), we get

$$
\left|\frac{\partial \tilde{\chi}_{w}}{\partial \sigma}\right| \leq C
$$

and in particular we have

$$
\left|\frac{\partial \tilde{\chi}_{w}}{\partial \sigma}(\sigma, 0)\right| \leq C
$$

Therefore the family of maps defined by

$$
\sigma \mapsto \tilde{\chi}_{w}(\sigma, 0)
$$

on $(-\infty, 0]$ into $M \subset T^{*} M$ is an equi-continuous family.

We now restrict $(9.23)$ to $s=0$ and rewrite it, by writing $\tilde{\chi}_{w}(\sigma, s)=(q(\sigma, s)$, $p(\sigma, s))$ as before, into

$$
\left\{\begin{array}{l}
\frac{\partial q}{\partial \sigma}+(T \pi \circ J) \nabla_{s} p+\operatorname{grad}\left(f_{i+1}-f_{i}\right)+T \pi\left(J_{\epsilon}-J\right)\left(\frac{\partial \tilde{\chi}_{w}}{\partial s}+X_{\left(F_{i+1}-F_{i}\right)}\left(\tilde{\chi}_{w}\right)\right)=0 \\
\frac{\partial q}{\partial s}-(T \pi \circ J) \nabla_{\sigma} p+T \pi \circ J\left(J_{\epsilon}-J\right)\left(\frac{\partial \tilde{\chi}_{w}}{\partial s}+X_{\left(F_{i+1}-F_{i}\right)}\left(\tilde{\chi}_{w}\right)\right)=0
\end{array}\right.
$$

Note that $p(\sigma, 0) \equiv 0$ and so $\nabla_{\sigma} p(\sigma, 0) \equiv 0$. Therefore from the second equation and (9.27), we have

$$
\left|\frac{\partial q}{\partial s}\right| \leq C\left|J_{\epsilon}-J\right|\left(\tilde{\chi}_{w}\right) \leq C \epsilon
$$

Then we have

$$
|\nabla s p| \leq|\nabla p|\left|\frac{\partial q}{\partial s}\right| \leq \widetilde{C} \epsilon
$$

because the boundedness of $|\nabla p|$ follows from the $C^{1}$-estimate (9.15) and from the definition (9.22) of $\tilde{\chi}_{w}$. By taking the $C^{1}$-limit of the first equation as $\epsilon_{j} \rightarrow 0$, any local limit of $\tilde{\chi}_{w}$, which we denote by $\chi_{\infty}$, satisfies the equation

$$
\frac{d \chi_{\infty}}{d \sigma}+\operatorname{grad}\left(f_{i+1}-f_{i}\right)\left(\chi_{\infty}\right)=0 .
$$

Since $M$ is compact, we may assume by taking a subsequence, that

$$
w(0,0) \rightarrow w(0) \rightarrow x \quad \text { as } \quad \epsilon_{j} \rightarrow 0 .
$$

Therefore the local limit of $\tilde{\chi}_{w}$ as $\epsilon \rightarrow 0$ satisfies the initial value problem

$$
\left\{\begin{array}{l}
\frac{d \chi}{d \sigma}+\operatorname{grad}\left(f_{i+1}-f_{i}\right)(\chi)=0 \\
\chi(0)=x
\end{array}\right.
$$

Furthermore, $\tilde{\chi}_{w_{j}}$ weakly converges to a cusp trajectory

$$
\chi_{0} \bigcup\left(\cup_{k=1}^{N} \chi_{k}\right)
$$

where

$$
\begin{aligned}
& \chi_{0}(0)=x, \lim _{\sigma \rightarrow-\infty} \chi_{0}(\sigma) \in \operatorname{Crit}\left(f_{2}-f_{1}\right) \\
& \chi_{k} \in \mathcal{M}\left(f_{i+1}-f_{i}\right) \text { for each } k=1, \cdots, N
\end{aligned}
$$

Now by an easy version of the gluing theorem, we conclude that there exists $\chi_{w} \in$ $\mathcal{M}(M: \vec{f}, \vec{p})$ such that

$$
d\left(\tilde{\chi}_{w_{j}}(\sigma, 0), \chi_{w}(\sigma)\right) \leq \frac{\delta}{3} .
$$

This finally finishes the proof of (9.24) and hence the proof of Theorem 9.1. 


\section{PART II. (HIGHER) MASSEY PRODUCT}

10. Moduli of metric ribbon trees and genus zero marked open Riemann surfaces. We are going to construct the natural stratification and the compactification of the moduli spaces $G r_{k}, \mathfrak{T}_{0, k}$ introduced in $\S 1$.

To explain our compactification of $\mathfrak{T}_{0, k}$, we use its relation to the moduli space of pointed genus 0 Riemann surface. We set

$$
\mathfrak{T}_{0, k}^{\mathbb{C}}=\frac{\left\{\left(z_{1}, \cdots, z_{k}\right) \mid z_{i} \in \mathbb{C} P^{1}, z_{i} \neq z_{j}, \quad \text { for } i \neq j\right\}}{\operatorname{PSL}(2 ; \mathbb{C})}
$$

Here $P S L(2 ; \mathbb{C})=\operatorname{Aut}\left(\mathbb{C} P^{1}\right)$ acts on $\left\{\left(z_{1}, \cdots, z_{k}\right) \mid z_{i} \in \mathbb{C} P^{1}, z_{i} \neq z_{j}, \quad\right.$ for $\left.i \neq j\right\}$ by $\underline{g\left(z_{1}, \cdots, z_{k}\right)}=\left(g z_{1}, \cdots, g z_{k}\right)$. We define an anti-holomorphic involution on $\mathfrak{T}_{0, k}^{\mathbb{C}}$ by $\overline{\left(z_{1}, \cdots, z_{k}\right)}=\left(\overline{z_{1}}, \cdots, \overline{z_{k}}\right)$. Let

$$
\mathfrak{T}_{0, k}^{\mathbb{R}}=\left\{x \in \mathfrak{T}_{0, k}^{\mathbb{C}} \mid \bar{x}=x\right\} .
$$

LEMMA 10.1. $\mathfrak{T}_{0, k}^{\mathbb{R}}$ consists of $(k-1)$ ! connected components. One of them is identified with $\mathfrak{T}_{0, k}$.

Proof. Let $x=\left[z_{1}, \cdots, z_{k}\right] \in \mathfrak{T}_{0, k}^{\mathbb{R}}$. By the abuse of notation, we write $x$ for the pointed space $\left[\mathbb{C} P^{1} ; z_{1}, \cdots, z_{k}\right]$ also. Composing the canonical anti-holomorphic diffeomorphism $x \rightarrow \bar{x}$ which is nothing but the identity map (note that as sets $x$ and $\bar{x}$ are the same), with the (holomorphic) isomorphism $x \rightarrow \bar{x}$ which is the involution mentioned above, we obtain an anti-holomorphic self-diffeomorphism $\tau: x \rightarrow x$. Since $x$ has no nontrivial automorphism, we have $\tau^{2}=1$. Therefore, the fixed point set of $\tau$ is biholomorphic to $S^{1}=\partial D^{2} \subset \mathbb{C} P^{1}$. We then find that $z_{i} \in \partial D^{2}$. We can fix the representative by taking $z_{1}=1, z_{2}=\sqrt{-1}$ and $z_{3}=-1$. The connected component of $\mathfrak{T}_{0, k}^{\mathbb{R}}$ is determined according to the (topological) position of $z_{i}$ 's, $i=4, \cdots, k$. By a simple combinatorial computation, we obtain the lemma.

Compactification of $\mathfrak{T}_{0, k}^{\mathbb{C}}$ has been studied extensively in algebraic geometry, and been used in the theory of quantum cohomology. Let us recall it briefly here. (See $[\mathrm{DM}]$ for a detailed exposition.)

A stable curve of genus zero $\left(\Sigma ; z_{1}, \cdots, z_{k}\right)$ consists of a connected and simply connected reduced curve $\Sigma$ with $k$ nonsingular marked points $z_{1}, \cdots, z_{k}$ such that $\Sigma$ has at worst an ordinary double point and each irreducible component of $\Sigma$ contains at least 3 points which are singular or marked. One can define a topology of the set $\overline{\mathfrak{T}_{0, k}^{\mathbb{C}}}$ of all stable curves of genus zero with $k$ marked points so that $\overline{\mathfrak{T}_{0, k}^{\mathbb{C}}}$ is a compactification of $\mathfrak{T}_{0, k}^{\mathbb{C}}$.

For each element $\left(\Sigma ; z_{1}, \cdots, z_{k}\right)$ of $\overline{\mathfrak{T}_{0, k}^{\mathbb{C}}}$, one can define its complex conjugate $\overline{\left(\Sigma ; z_{1}, \cdots, z_{k}\right)}$ such that the map $\left(\Sigma ; z_{1}, \cdots, z_{k}\right) \mapsto \overline{\left(\Sigma ; z_{1}, \cdots, z_{k}\right)}$ is an extension of the map $\left(\mathbb{C} P^{1} ; z_{1}, \cdots, z_{k}\right) \mapsto\left(\mathbb{C} P^{1} ; \overline{z_{1}}, \cdots, \overline{z_{k}}\right)$, which is defined on $\mathfrak{T}_{0, k}^{\mathbb{C}}$. There is a canonical anti-holomorphic diffeomorphism $:\left(\Sigma ; z_{1}, \cdots, z_{k}\right) \rightarrow \overline{\left(\Sigma ; z_{1}, \cdots, z_{k}\right)}$. We set

$$
\overline{\mathfrak{T}}_{0, k}^{\mathbb{R}}=\left\{x \in \overline{\mathfrak{T}}_{0, k}^{\mathbb{C}} \mid \bar{x}=x\right\}
$$

Let $\overline{\mathfrak{T}}_{0, k}$ be the closure of $\mathfrak{T}_{0, k}$ in $\overline{\mathfrak{T}}_{0, k}^{\mathbb{C}}$. It is contained in $\overline{\mathfrak{T}}_{0, k}^{\mathbb{R}}$. For an element $\left(\Sigma ; z_{1}, \cdots, z_{k}\right)$ of $\overline{\mathfrak{T}}_{0, n}$ we have an isomorphism $\overline{\left(\Sigma ; z_{1}, \cdots, z_{k}\right)} \rightarrow\left(\Sigma ; z_{1}, \cdots, z_{k}\right)$. By the stability (namely the nonexistence of nontrivial automorphism of an element of $\overline{\mathfrak{T}}_{0, k}^{\mathbb{C}}$ ), this isomorphism is unique. Hence by an argument similar to the 


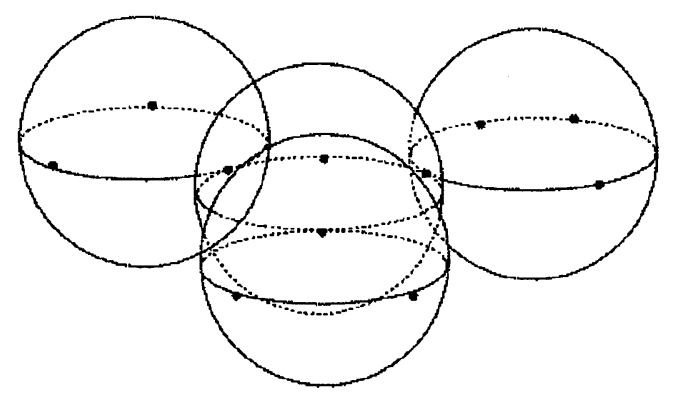

$\Sigma$

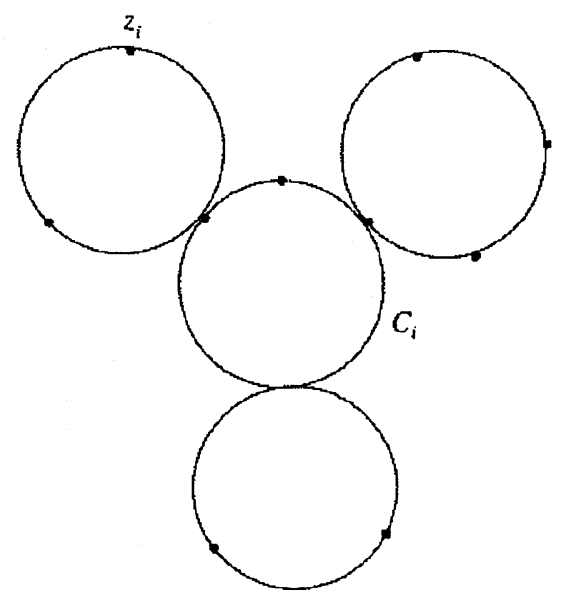

Figure 10.1

proof of Lemma 10.1, we obtain an anti-holomorphic involution $\tau:\left(\Sigma ; z_{1}, \cdots, z_{k}\right) \rightarrow$ $\left(\Sigma ; z_{1}, \cdots, z_{k}\right)$. The points $z_{i}$ are fixed by this involution. Let

$$
C=\{z \in \Sigma \mid \tau(z)=z\}
$$

$C$ is a union of finitely many circles patched at finitely many points, and $z_{i}$ are contained in $C$. (Figure 10.1).

We define a one dimensional simplicial complex $T$ from $C$ as follows. The vertex of $T$ corresponds to a circle of $C$ or one of the points $z_{i}$. Let $\left\{v_{1}, \cdots, v_{m}\right\} \cup\left\{z_{1}, \cdots, z_{k}\right\}$ be the set of vertices. We join two vertex $v_{i}$ and $v_{j}$ if corresponding circles intersect to each other in $C$. We join $v_{i}$ and $z_{j}$ if the circle corresponding to $v_{i}$ contains $z_{j}$. We never join $z_{i}$ and $z_{j}$. (Figure 10.1)

LEMMA 10.2. $T$ is simply connected.

Proof. If not, we can find a nontrivial loop $S^{1}$ in $T$. It is easy to see that we can lift it to the nontrivial loop of $\Sigma$. But $\Sigma$ is simply connected by assumption, which gives rise to a contradiction.

For each vertex $v_{i}$ in $T$, we have a cyclic order of the edges containing $v_{i}$. This cyclic order is induced by the (counter clockwise) cyclic order of the circles corresponding to $v_{i}$. We recall that a tree with fixed cyclic order of the set of edges of each vertex, has a unique embedding into $\mathbb{R}^{2}$ such that the cyclic order is compatible to 
the orientation of $\mathbb{R}^{2}$. Thus, for each element of $\overline{\mathfrak{T}}_{0, k}$ we obtain an element $\left(T, i, z_{1}\right)$ of $G_{k}$ such that exterior vertices correspond to $z_{1}, \cdots, z_{k}$. Let $\mathfrak{T}(\mathfrak{t})$ be the set of all elements of $\overline{\mathfrak{T}}_{0, k}$ such that the graph we found above is $t$.

We thus have described the relation between our two moduli spaces. To make them more explicit, we are going to construct a map $\Theta: G r_{k} \rightarrow \mathfrak{T}_{0, k}$

Definition 10.3. Let $\mathfrak{t} \in G_{k}, \ell: C_{\text {int }}^{0}(T) \rightarrow \mathbb{R}_{4}$. We take an Euclidean rectangle $L_{e}=[0, \ell(e)] \times[0,1]$ for each $e \in C_{\text {int }}^{1}(\mathfrak{t})$, and $L_{e}=(-\infty, 0] \times[0,1]$ for each $e \in C_{\text {ext }}^{1}(\mathfrak{t})$. We remove $\partial[0, \ell(e)] \times\{1 / 2\}$ or $\partial(-\infty, 0] \times\{1 / 2\}$ from $L_{e}$. Let $v \in C_{\text {int }}^{0}(\mathfrak{t})$. We consider edges $e, e^{\prime}$ such that $v \in \partial e, \partial e^{\prime}$. We assume that $e^{\prime}$ is the next edge to $e$ according to the cyclic order we put on the set of the edges containing $v$. Then, in the case when the orientation of $e, e^{\prime}$ goes from $v$ to another edge, we glue $\{0\} \times(1 / 2,1] \subset \partial L_{e}$ and $\{0\} \times[0,1 / 2) \subset \partial L_{e}^{\prime}$. If the orientation is different we glue in a similar way. (See Figure 10.2).

We thus obtain a space $X_{0}(\mathfrak{t}, \ell)$ together with an (incomplete) flat metric on it. We would like to note that in the metric point of view, $X_{0}(t, \ell)$ is not a sub-domain of $\mathbb{C}$. $X_{0}(t, \ell)$ has holes corresponding to each of the interior vertex of $T$. We can fill these holes conformally, and obtain a space $X(\mathfrak{t}, \ell)$ equipped with a complex structure. This space has $k$ boundary components and $k$ ends. Therefore we have produced an element of $\mathfrak{T}_{0, k}$ for each $\ell \in G r_{k}$. We denote this element by $\Theta(\ell)$.

It is easy to see that the assignment $\ell \mapsto \Theta(\ell)$ defines a continuous map, which we denote by $\Theta: G r_{k} \rightarrow \mathfrak{T}_{0, k}$.

THEOREM 10.4. $\Theta: G r_{k} \rightarrow \mathfrak{T}_{0, k}$ is a homeomorphism.

We will prove this theorem in $\S 14$. Theorem 10.4 is closely related to the theory of quadratic differentials ([Str]).

Now we construct an open covering $\cup_{\mathfrak{t} \in G_{k}} U(\mathfrak{t}: \epsilon, \alpha)=G r_{k}$ which will be used in the later sections. We introduce some notations first.

DEFINITION 10.5.

(i) Let $\mathfrak{t}, \mathfrak{t}^{\prime} \in G_{k}$. We say $\mathfrak{t} \succ \mathfrak{t}^{\prime}$ if and only if $\mathfrak{t}^{\prime}$ is obtained by collapsing some edges of $t$.

(ii) When $\mathfrak{t} \succ \mathfrak{t}^{\prime}$, we have a surjective map $\pi: T \rightarrow T^{\prime}$. For $v \in C_{\text {int }}^{0}\left(\mathfrak{t}^{\prime}\right)$, we put $\overline{\mathfrak{c}}_{v}=\pi^{-1}(v)$ which is a subgraph of $T$. We identify each edge $e$ of $\mathfrak{t}^{\prime}$ with an edge $\pi^{-1}(e)$ of $T$.

(iii) For each $\overline{\mathfrak{c}}_{v}$, we consider the edges in $\mathfrak{t}$ that intersect but are not contained in $\overline{\mathfrak{c}}_{v}$. For each such edge, we attach an exterior edge $(-\infty, 0]$ to $\overline{\mathfrak{c}}_{v}$ at the point where the edge intersects $\overline{\mathfrak{c}}_{v}$. Let $\mathfrak{c}_{v}$ be the graph obtained in this way.

Now let $\alpha>0$ and $\epsilon>0$. We define

$$
U\left(\mathfrak{t}^{\prime}: \epsilon, \alpha\right)=\left\{\begin{array}{l|l}
(\ell) \in G r_{k} & \begin{array}{l}
\ell \in G r(\mathfrak{t}), \mathfrak{t} \succ \mathfrak{t}^{\prime} \\
\ell(e)>\epsilon^{-\alpha}, \quad \text { if } e \in C_{i n t}^{1}\left(\mathfrak{t}^{\prime}\right) \\
\ell(e) \leq \epsilon^{-\alpha}, \text { if } e \notin C_{i n t}^{1}\left(\mathfrak{t}^{\prime}\right)
\end{array}
\end{array}\right\}
$$

We will choose the constants $\alpha=\alpha(\mathfrak{t})$ so that $0<\alpha(\mathfrak{t})<1$ and $\frac{\alpha\left(\mathfrak{t}^{\prime}\right)}{\alpha(\mathfrak{t})}<<1$ if $\mathfrak{t} \succ \mathfrak{t}^{\prime}$. Then it is easy to see that

$$
\bigcup_{\mathfrak{t} \in G_{k}} U(\mathfrak{t}: \epsilon, \alpha(\mathfrak{t}))=G r_{k}
$$

is an open covering of $G r_{k}$.

11. Construction of approximate solutions. We now begin with the proof 

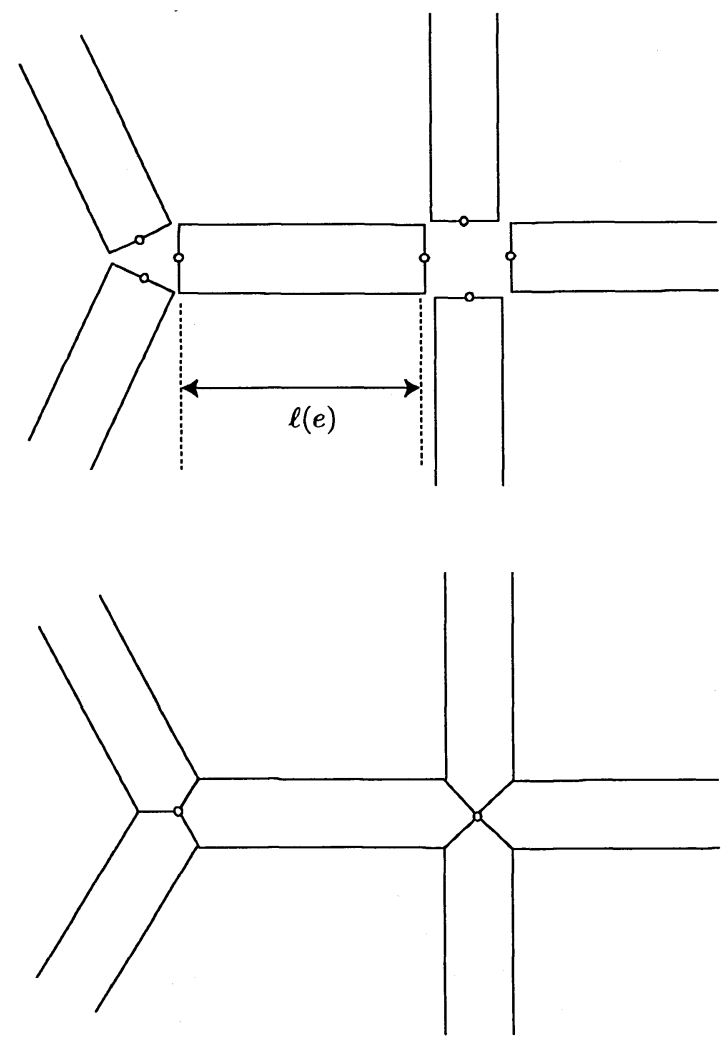

Figure 10.2

of our main theorem, Theorem 1.7 for general $k$. The proof goes along a similar line to that of Part I. In fact the construction of our open covering of the moduli space $G r_{k}$ in $\S 10$ has been organized so that it works well with the argument of Part I.

To imitate Part I, we first need an analogy of Proposition 4.1 for general $k$. To state it we need some notations. Let $a_{1}, \cdots, a_{k} \in \mathbb{R}^{n}$. Suppose that each of subsets of $\left\{a_{1}, \cdots, a_{k}\right\}$ that consist of $k-1$ elements is linearly independent. We put $\Lambda_{i}=\left\{\left(x+\sqrt{-1} a_{i} \mid x \in \mathbb{R}^{n}\right\} \subset \mathbb{C}^{n}\right.$.

Next let $z=\left[z_{1}, \cdots, z_{k}\right] \in \mathfrak{T}_{0, k}$. Choose a conformal diffeomorphism of $D^{2}-$ $\left\{z_{1}, \cdots, z_{k}\right\}$ to an open subset $\Theta(z)$ of $\mathbb{C}$. We take $\Theta(z)$ so that there is a compact subset $\Theta_{0}(z)$ such that

$$
\Theta(z)-\Theta_{0}(z)=\bigcup_{i=1}^{k} \Theta_{i}(z)
$$

and $\Theta_{i}(z)$ is isometric to $(-\infty, 0] \times[0,1]$ where $\Theta_{i}(z)$ is the end corresponding to $z_{i}$. We let $\partial_{i} \Theta(z)$ be the connected component of $\partial \Theta(z)$ corresponding to $\partial_{i} D^{2}$.

Proposition 11.1. There is a holomorphic map

$$
\widetilde{w}_{z}: \Theta(z) \rightarrow \mathbb{C}^{n}
$$

such that

$$
\widetilde{w}_{z}\left(\partial_{i} \Theta(z)\right) \subset \Lambda_{i}
$$




$$
\lim _{\tau \rightarrow-\infty}\left(\left.\operatorname{Im} \widetilde{w}_{z}\right|_{\Theta_{i}}(\tau, t)-\left(t\left(a_{i+1}-a_{i}\right)+a_{i}\right)=0\right.
$$

Such a map is unique up to addition of real constant vectors.

Proof. The proof of Proposition 11.1 is a straight forward generalization of the proof of Proposition 4.1 in $\S 4$. So we discuss the proof only briefly. We may assume that $n=k-1$ and $a_{i}$ generates $\mathbb{C}^{n}$. We then can take $k$ linear maps $\pi_{i}: \mathbb{C}^{n} \rightarrow \mathbb{C}$ such that

(11.2.1) $\pi_{i}$ is linear and defined over $\mathbb{R}$.

(11.2.2) $\pi_{i}\left(a_{j}\right)=-1$ if $j \neq i$.

$(11.2 .3) \pi_{i}\left(a_{i}\right)=1$.

It follows that $\left(\pi_{1}, \cdots, \pi_{k-1}\right)$ is a linear isomorphism. We have

$$
\begin{aligned}
& \pi_{i}\left(\Lambda_{j}\right)=\{x-\sqrt{-1} \mid x \in \mathbb{R}\} \quad \text { for } j \neq i . \\
& \pi_{i}\left(\Lambda_{i}\right)=\{x+\sqrt{-1} \mid x \in \mathbb{R}\}
\end{aligned}
$$

By the Riemann mapping theorem, we can find a holomorphic map $\widetilde{w}_{i}: \Theta_{z} \rightarrow \mathbb{C}$ such that

$$
\widetilde{w}_{i}\left(\partial_{j} \Theta(z)\right) \subset \pi_{i}\left(\Lambda_{j}\right) .
$$

We then put $\widetilde{w}_{z}=\left(\pi_{1}, \cdots, \pi_{k-1}\right)^{-1}\left(\widetilde{w}_{1}, \cdots, \widetilde{w}_{k-1}\right)$.

The proof that $\widetilde{w}_{z}$ has required property is the same as that of Proposition 4.1 and so omitted. The proof of uniqueness is also similar.

In $\S 17$, we will specify how to remove the ambiguity in Proposition 11.1.

We will apply this proposition as we applied Proposition 4.1 in Part I. To do this, we need some preliminaries. Let $\widehat{\mathfrak{t}} \succ \mathfrak{t}$. Then, for each vertex $v$ of $\mathfrak{t}$, we have $\mathfrak{c}_{v} \in G_{k_{v}}$ as in Definition 10.5. Here $k_{v}$ denotes the number of edges containing $v$. If $\ell \in G r(\mathfrak{t})$, it induces $\ell_{v} \in G r\left(\mathfrak{c}_{v}\right)$, hence an element $\Theta\left(\ell_{v}\right)$ of $\mathfrak{T}_{0, k_{v}}$.

By the construction of Definition 10.3, $\Theta(\ell) \in \mathfrak{T}_{0, k}$, together with its explicit coordinate, can be obtained from $\Theta\left(\ell_{v}\right)$ 's as follows: Each of the ends of $\Theta\left(\ell_{v}\right)$ is isometric to $(-\infty, 0] \times[0,1]$, and the ends of $\Theta\left(\ell_{v}\right)$ correspond one to one to the edges of $t$ containing $v$. We remove $\left(-\infty,-\epsilon^{-\alpha} / 3\right] \times[0,1]$ from each of these ends. We place these domain at the position $v$.

Next, we take a rectangle $\left[\epsilon^{1-\alpha} / 6, \ell(e)-\epsilon^{1-\alpha} / 6\right] \times[0,1]$ for each $e \in C_{\text {int }}^{1}(\mathfrak{t})$ and place it at the position of the edge $e$. If $v \in \partial e$, and the orientation of $e$ goes from $v$ to another vertex, we identify $\left[\ell(e)-\epsilon^{1-\alpha} / 3, \ell(e)-\epsilon^{1-\alpha} / 6\right] \times[0,1]$ with $\left[-\epsilon^{1-\alpha} / 3,-\epsilon^{1-\alpha} / 6\right] \times[0,1] \subset \Theta\left(\ell_{v}\right)$. If the orientation is different we glue in a similar way. We have thus obtained a space with complex structure. It is immediate to see from the definition that this space coincides with $\Theta(\ell)$.

Now we outline the construction of approximate solutions. We first consider the re-scaling map $\pi_{\epsilon}: G r_{k} \rightarrow G r_{k}$ defined by

$$
\pi_{\epsilon}(\ell)(e)=\frac{\ell(e)}{\epsilon} .
$$

Note that this is a diffeomorphism which preserves each stratum. We will define our approximate solutions on $\Theta\left(\frac{\ell}{\epsilon}\right)$. As in Part I, we decompose $\Theta(\ell)$ into the "neck" regions and the "center" regions around the vertices. First we use Proposition 11.1 via the exponential map to construct approximate solutions of the pseudo-holomorphic map equation on $\Theta\left(\ell_{v}\right)$ into $T^{*} M$. On the neck regions (i.e., rectangle regions) $\left[\epsilon^{-\alpha} / 6, \frac{\ell(e)}{\epsilon}-\epsilon^{-\alpha} / 6\right] \times[0,1]$, we use the gradient lines as in Part I to define them. 


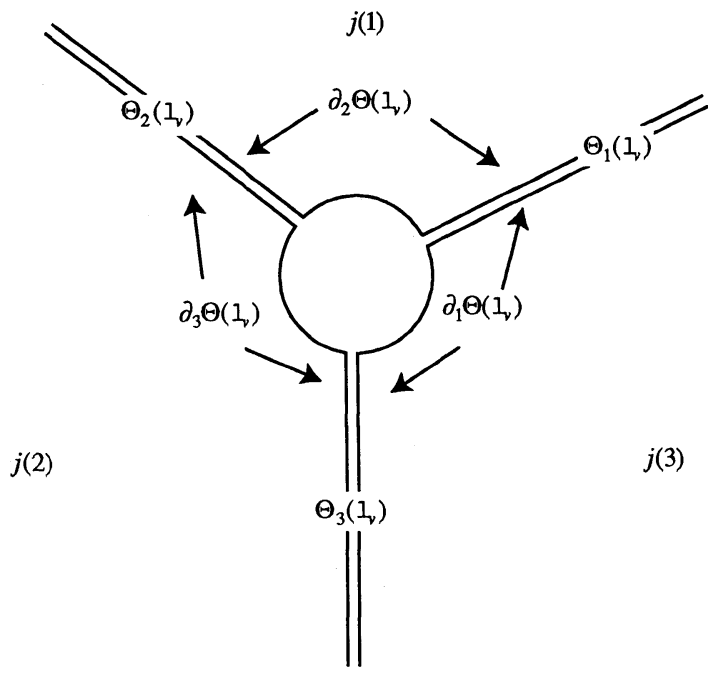

FIGURE 11.1

By gluing these using the partitions of unity, we will obtain approximate solutions. Making this construction precise is the goal of this section.

We first need to remark about some technical trouble which was not present in the case of $k=3$ in Part I: The family of domains $\Theta\left(\ell_{v}\right)$ form a non-compact family as $\ell_{v}$ varies and $\epsilon$ goes to 0 . Therefore it is not clear that the convergence in (11.1.2) can be made uniform as $\epsilon$ goes to 0 . We will discuss this trouble in $\S 16$ in more detail. For the moment, we just state and use one lemma (Lemma 11.2) which will be used in our construction below.

To state this lemma we need some notations. Let us number the set of edges $e$ such that $v \in \partial e$ in a way compatible to the cyclic order. We put $\operatorname{leg}\left(e_{i}\right)=j(i)=r i g\left(e_{i+1}\right)$. Corresponding to each exterior edge $e_{i}$ of $\ell$, we have the exterior end of $\Theta\left(\ell_{v}\right)$, which we denote by $\Theta_{i}\left(\ell_{v}\right)$. We use $(\tau, t) \in(-\infty, 0]$ for its coordinate. Denote by $\partial_{i} \Theta\left(\ell_{v}\right)$ the component of the boundary $\partial \Theta(\ell)$ such that

$$
\begin{gathered}
\partial_{i} \Theta\left(\ell_{v}\right) \cap \Theta_{i}\left(\ell_{v}\right) \neq \emptyset \\
\partial_{i} \Theta\left(\ell_{v}\right) \cap \Theta_{i+1}\left(\ell_{v}\right) \neq \emptyset
\end{gathered}
$$

(See Figure 11.1).

Let $p \in M$ and denote $a_{i}=\operatorname{grad}_{p} f_{j(i)}$, and

$$
\Lambda_{i}=\left\{x+\sqrt{-1} a_{i} \mid x \in \mathbb{R}^{n}\right\} \subset \mathbb{C}^{n}=T_{p} M \otimes \mathbb{C} .
$$

LEMmA 11.2. Let $\Theta\left(\ell_{v}\right)$ be as above and $\widetilde{w}_{v}: \Theta\left(\ell_{v}\right) \rightarrow \mathbb{C}^{n}$ be the holomorphic map obtained by using Proposition 11.1. Namely

(11.3.1) $\widetilde{w}_{v}\left(\partial_{i} \widetilde{w}_{v}\right) \subset \Lambda_{i}$.

$$
\lim _{\tau \rightarrow-\infty}\left(\left.\operatorname{Im} \widetilde{w}_{v}\right|_{\Theta_{i}}(\tau, t)-\left(t\left(a_{i+1}-a_{i}\right)+a_{i}\right)=0\right.
$$

Then the convergence in (11.4) is uniform on $\epsilon, p$ and $\ell_{v}$.

The proof of Lemma 11.2 will be postponed until $\S 16$. 
Now we describe the appropriate space of maps where we do the necessary estimates. First recall that to each element $\ell \in U(\mathfrak{t}: \epsilon, \alpha)$ is associated the domain $\Theta\left(\frac{\ell}{\epsilon}\right)$ with the complex structure that is induced from the flat metric (with finitely many singularities), which represent it (see the paragraph right before Theorem 10.4). These will be the spaces where we do all the estimates implicit below. We denote by

$$
M a p_{U(\mathfrak{t}: \epsilon, \alpha)}\left(T^{*} M: \vec{\Lambda}^{\epsilon}, \vec{p}^{\epsilon}\right)=\bigcup_{\ell \in U(\mathfrak{t}: \epsilon, \alpha)} \operatorname{Map}_{\underline{\epsilon}}\left(T^{*} M: \vec{\Lambda}^{\epsilon}, \vec{p}^{\epsilon}\right)
$$

the fiber bundle over $U(\mathfrak{t}: \epsilon, \alpha)$, whose fiber at $\ell$ is given by

$$
\begin{aligned}
\operatorname{Map}_{\underline{\ell}}\left(T^{*} M: \overrightarrow{\Lambda^{\epsilon}}, \overrightarrow{p^{\epsilon}}\right) & =\left\{w: \Theta\left(\frac{\ell}{\epsilon}\right) \rightarrow T^{*} M \mid w\right. \text { is a smooth map } \\
& \text { satisfying (1.3.1) and (1.3.2) }\} .
\end{aligned}
$$

We would like to mention that $w$ in this set does not necessarily satisfy (1.3.3). We denote by $\|\cdot\|_{0, p, \epsilon}$ the obvious weighted Sobolev norm on the space $\operatorname{Map}_{\gamma}\left(T^{*} M\right.$ : $\left.\vec{\Lambda}^{\epsilon}, \vec{p}^{\epsilon}\right)$ in terms of the induced metric on $\Theta(\gamma)$. We would like to point out that the (ordinary) Sobolev constant of the domains $\frac{\ell}{\epsilon}$ are uniform over $\ell \in U(\mathfrak{t}: \epsilon, \alpha)$ and $\epsilon \rightarrow 0$.

We then consider the fibre product $U(\mathfrak{t}: \epsilon, \alpha) \times_{\pi_{\epsilon}} \mathcal{M}_{g}(M: \vec{f}, \vec{p})$ using the map $\pi_{\epsilon}: U(\mathfrak{t}: \epsilon, \alpha) \rightarrow G r_{k}$.

Definition 11.3. $\operatorname{Map}\left(T^{*} M: \vec{\Lambda}^{\epsilon}, \vec{p}^{t}\right)$ be the set of all pairs $\left(\left[z_{1}, \cdots, z_{k}\right], \omega\right)$, such that $\left[z_{1}, \cdots, z_{k}\right] \in \mathfrak{T}_{0, k}$ and $\omega: D^{2} \rightarrow T^{*} M$ is a smooth map satisfying (1.3.1), (1.3.2) but not necessary (1.3.3). Note that it follows that $\operatorname{Map}_{U(\mathfrak{t}: \epsilon, \alpha)}\left(T^{*} M, \overrightarrow{\Lambda^{\epsilon}}, \vec{p}\right)$ is a subset of $\operatorname{Map}\left(T^{*} M: \vec{\Lambda}^{\epsilon}, \vec{p}^{\epsilon}\right)$.

Now our main result of this section is the following.

Proposition 11.4. There exists a constant $C>0$ independent of $\epsilon$, and maps

$$
\Psi_{\mathfrak{t}, \alpha}^{\epsilon}: U(\mathfrak{t}: \epsilon, \alpha) \times_{\pi_{\epsilon}} \mathcal{M}_{g}(M ; \vec{f}, \vec{p}) \rightarrow \operatorname{Map}_{U(\mathfrak{t}: \epsilon, \alpha)}\left(T^{*} M: \vec{\Lambda}^{\epsilon}, \vec{p}^{\epsilon}\right)
$$

such that the followings are satisfied:

(11.5.1) If $\omega$ is in the image of $\Psi_{\mathfrak{t}, \alpha}^{\epsilon}$ then we have

$$
\left\|\bar{\partial}_{J} \omega\right\|_{0, p, \epsilon} \leq C \epsilon^{\frac{2+(p-1) \alpha}{p}} \text {. }
$$

(11.5.2) The following Figure 11.2 commutes.

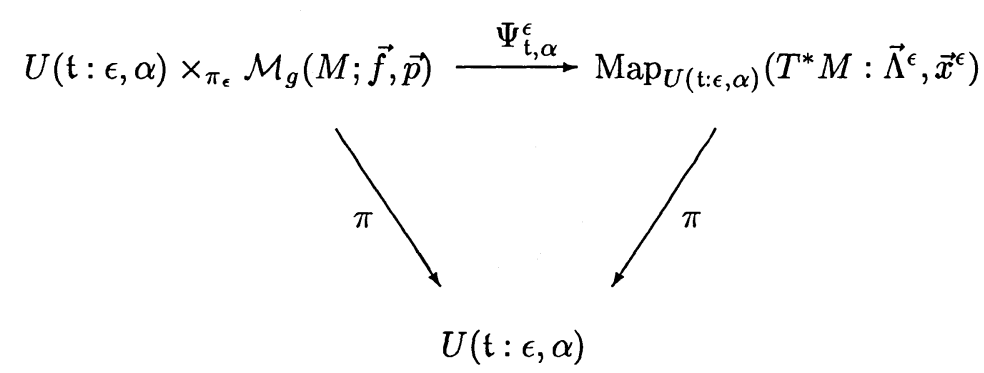

Figure 11.2

Proof. Let $\ell \in U(\mathfrak{t}: \epsilon, \alpha) \cap G r(\mathfrak{t})$ for some $\widehat{\mathfrak{t}} \succ \mathfrak{t}$ and $I \in \mathcal{M}_{g}(M ; \vec{f}, \vec{p})$ such that $\pi_{\epsilon}(\ell)=\pi(I)$. Here $\pi: \mathcal{M}_{g}(M ; \vec{f}, \vec{p}) \rightarrow G r_{k}$ is the projection. We decompose the 
domain $\Theta(\ell)$ as follows. We put

$$
\Theta_{e} \simeq[0, \ell(e)] \times[0,1] \subset \Theta(\ell)
$$

for $e \in C_{\text {int }}^{1}(\mathfrak{t})$ and

$$
\Theta_{e} \simeq(-\infty, 0] \times[0,1] \subset \Theta(\ell)
$$

for $e \in C_{e x t}^{1}(\mathfrak{t})$.

Let $v \in C_{\text {int }}^{0}(\mathfrak{t})$. We take a vertex $v_{0} \in \mathfrak{c}_{v}$ and put $p_{v}=I\left(v_{0}\right) \in M$. (The precise choice will be discussed in more detail in $\S 17$.) By Lemma 11.2, we obtain a map

$$
\widetilde{w}_{v} \rightarrow T_{p_{v}} M \otimes \mathbb{C}
$$

where we identify

$$
T_{p_{v}} M \otimes \mathbb{C}=T_{\left(p_{v}, 0\right)}\left(T^{*} M\right)
$$

Let $e \in C^{1}(\mathfrak{t})$. Let

$$
\chi_{e}:\left[0, \frac{\ell(e)}{\epsilon}\right] \rightarrow M
$$

or

$$
\chi_{e}:(-\infty, 0] \rightarrow M
$$

be the restriction of $I$ to the edge $e . \chi_{e}$ is a gradient line of $-\left(f_{l e f(e)}-f_{r i g(e)}\right)$. We put

$$
\Theta_{e}\left(\frac{C}{\epsilon^{\alpha}}\right)=\left[C \epsilon^{-\alpha}, \frac{\ell(e)}{\epsilon}-C \epsilon^{-\alpha}\right] \times[0,1] \subset \Theta_{e}
$$

when $e \in C_{\text {int }}^{1}(\mathfrak{t})$, and

$$
\Theta_{e}\left(\frac{C}{\epsilon^{\alpha}}\right)=\left(-\infty,-C \epsilon^{-\alpha}\right] \times[0,1] \subset \Theta_{e}
$$

when $e \in C_{e x t}^{1}(\mathfrak{t})$.

Then for each $v \in C_{\text {int }}^{0}(\mathfrak{t})$, we put

$$
w_{v}^{\epsilon}(z)=\exp _{p_{v}}\left(\epsilon \widetilde{w}_{v}(z)\right)
$$

on

$$
z \in \Theta\left(\ell_{v}\right)-\bigcup\left(-\infty,-\epsilon^{-\alpha} / 6\right] \times[0,1] .
$$

For $e \in C^{1}(\mathfrak{t})$, we put

$$
w_{e}^{\epsilon}(\tau, t)=\psi_{\epsilon t}^{f_{\text {leg(e) }}} \circ \psi_{\epsilon(1-t)}^{f_{\text {rig(e) }}}\left(\chi_{e}(\epsilon \tau)\right)
$$

on $\Theta_{e}\left(\frac{1}{3 \epsilon^{\alpha}}\right)$. On $\Theta_{e}\left(\frac{1}{6 \epsilon^{\alpha}}, \frac{1}{3 \epsilon^{\alpha}}\right):=\Theta_{e}\left(\frac{1}{3 \epsilon^{\alpha}}\right)-\Theta_{e}\left(\frac{1}{6 \epsilon^{\alpha}}\right)$ we use a partition of unity in exactly the same way as $\S 4$, to patch (11.6) and (11.7). Thus we have obtained a map :

$$
w^{\epsilon}=w^{\epsilon, I}: \Theta\left(\frac{\ell}{\epsilon}\right) \rightarrow T^{*} M
$$

We put

$$
\Psi_{\mathfrak{t}, \alpha}^{\epsilon}(\ell, I)=w^{\epsilon, I} .
$$

The commutativity of the Figure 11.2 is an immediate consequence of the construction.

To prove the estimates (11.5.1) repeating those in Part I, we need to prove the following lemma which is relevant to prove the analogoues of (5.6) and (5.8) there. 
Once this lemma is proved, (11.5.1) can be proved in the same way as in Part I. We remark that we also need this lemma to show that $C$ in (11.5.1) is independent of $\epsilon$.

LEMMA 11.5. We can choose $\widetilde{w}_{v}$ so that the following holds: Let $(\tau, t) \in \Theta_{e}\left(\frac{1}{6 \epsilon^{\alpha}}\right.$, $\left.\frac{1}{3 \epsilon^{\alpha}}\right)$. Then

$$
\operatorname{dist}\left(w_{v}^{\epsilon}(\tau, t), w_{e}(\tau, t)\right)<C \max \left\{\epsilon, \epsilon^{2(1-\alpha)}\right\}
$$

Here $v$ is the vertex corresponding to $0 \in[0, \ell(e)] \simeq e$.

We postpone the proof of Lemma 11.5 until $\S 17$.

12. Transversality of the graph flows. We constructed in $\S 11$ an approximate solution corresponding to each element of $\mathcal{M}(M: \vec{f}, \vec{p})$. Our next step is to modify it and to find an exact solution nearby of the pseudo-holomorphic map equation (or the (nonlinear) Cauchy-Riemann equation). Roughly speaking, we can carry out this modification, provided the linearized operator is surjective (namely when there exists a right inverse of the operator.) This is the way how we did in the case when $k=3$ in $\S 6,7$. The existence of the right inverse was proved there by making use of the transversality hypothesis in Theorem 3.1 for the unstable manifolds of gradient vector fields.

To generalize the line of ideas in $\S 6,7$ to general $k$, we need to define and verify the transversality of the moduli space $\mathcal{M}_{g}(M: \vec{f}, \vec{p})$ and to understand its relation to the existence of a right inverse of the linearization of the Cauchy-Riemann equation. This is the analogoue to the proof of Theorem 1.4. But we will not complete the proof of this theorem at this stage, since we still have to incorporate and glue the moduli spaces corresponding to different combinatorial types of graphs. Therefore in this section, we fix the combinatorial type of $t$ and consider the subset $G r(t)$ of $G r_{k}$ and study the transversality there. The gluing construction we need to complete the proof of Theorem 1.4 will be explained in $\S 14$ and $\S 15$.

Let $\mathfrak{t}=(T, i, p)$. We fix a vertex $v_{0} \in C_{i n t}^{0}(T)$. For each exterior edge $e_{i}(i=$ $1, \cdots, k$ ), let $v_{i}$ be the interior vertex contained in $e_{i}$. (The other vertex of $e_{i}$ is exterior.) We remark that $v_{i}=v_{j}$ may happen for $i \neq j$. We order them so that

$$
v_{1}=\cdots=v_{i_{1}-1} \neq v_{i_{1}}=\cdots=v_{i_{2}-1} \neq v_{i_{2}}=\cdots \neq v_{i_{m}}=\cdots=v_{k} .
$$

Here $i_{1}<i_{2} \cdots<i_{m}$. We are going to define a map

$$
\operatorname{Exp}=\operatorname{Exp}_{\mathfrak{t}}: M \times G r(\mathfrak{t}) \rightarrow M^{m} .
$$

For $1 \leq h \leq m$ there is a unique minimal path in $T$ joining $v_{0}$ and $v_{i_{h}}$. Let $e_{j_{1}^{h}}, \ldots$, $e_{j_{c_{h}}^{h}}$ be the edges contained in this path in this order, see Figure 12.1.

Let $V_{b}^{h}$ be the vector field such that

$$
V_{b}^{h}=-\left(\operatorname{grad} f_{l e f\left(e_{j_{b}^{h}}\right)}-\operatorname{grad} f_{r i g\left(e_{j_{b}^{h}}\right)}\right)
$$

Let $\operatorname{Exp}\left(t V_{b}^{h}\right): M \rightarrow M$ be the one parameter group of transformations associated to this vector field. We denote

$$
\operatorname{Exp}_{h}(p, \ell)=\operatorname{Exp}\left(\ell\left(e_{j_{1}^{h}}\right) V_{1}^{h}\right) \circ \cdots \circ \operatorname{Exp}\left(\ell\left(e_{j_{c_{h}}^{h}}\right) V_{c_{h}}^{h}\right)(p)
$$

In other words, we define a map

$$
I: T-\bigcup_{v \in C_{e x t}^{0}(T)}\{v\} \rightarrow M
$$




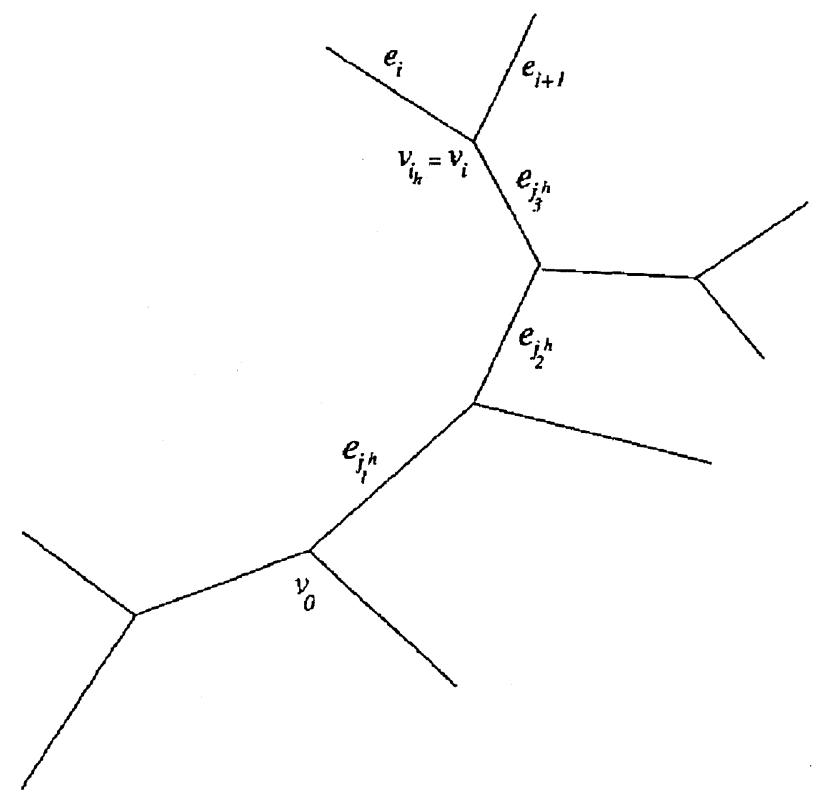

Figure 12.1

such that $I$ satisfies (1.4.2), (1.4.3) and $I\left(v_{0}\right)=p$, and put :

$$
I\left(v_{i_{h}}\right)=\operatorname{Exp}_{h}(p, \ell) .
$$

We set

$$
\operatorname{Exp}(p, \ell)=\left(\operatorname{Exp}_{1}(p, \ell), \cdots, \operatorname{Exp}_{m}(p, \ell)\right) .
$$

We write $\operatorname{Exp}_{\vec{f}}$ in case we need to specify $\vec{f}$.

For a critical point $p_{i}$ of $f_{i+1}-f_{i}$, let $W_{p_{i}}^{-}\left(f_{i+1}-f_{i}\right)$ be the unstable manifold of the gradient vector field of $f_{i+1}-f_{i}$. Let $\pi: \mathcal{M}_{g}(M: \vec{f}, \vec{p}) \rightarrow G r_{k}$ be the natural projection. We denote by $\mathcal{M}_{g}(M: \vec{f}, \vec{p}, \mathfrak{t})$ the inverse image of $\operatorname{Gr}(\mathfrak{t})$ in $\mathcal{M}_{g}(M: \vec{f}, \vec{p})$ under the map $\pi$. Now the following lemma is immediate from the definition.

LEMMA 12.1. There exists a natural one to one correspondence

$$
\operatorname{Exp}^{-1}\left(\prod_{h=1}^{m} \bigcap_{j=i_{h}}^{i_{h+1}-1} W_{p_{j}}^{-}\left(f_{j+1}-f_{j}\right)\right) \simeq \mathcal{M}_{g}(M: \vec{f}, \vec{p}, \mathfrak{t})
$$

Now we would like to formulate the transversality of the moduli space $\mathcal{M}_{g}(M$ : $\vec{f}, \vec{p}, \mathfrak{t})$. To study the transversality property of $\mathcal{M}_{g}(M: \vec{f}, \vec{p})$, the identification in Lemma 12.1 is not suitable enough because the structure of the set $\cap_{j=i_{h}}^{i_{h+1}-1} W_{p_{j}}^{-}\left(f_{j+1}-\right.$ $f_{j}$ ) could be quite complicated. For example there is no simple criterion for the set to be a smooth submanifold unless the intersection is that of a pair of submanifolds. Because of this reason, we consider the transversality in the following way: We assume that all the functions $f_{j+1}-f_{j}$ are of the Morse-Smale type and so all $W_{p_{j}}^{-}\left(f_{j+1}-f_{j}\right)$ 
are smooth submanifolds and intersect one another transversely. We study the map

$$
E X P: M \times G r(\mathfrak{t}) \times \prod_{h=1}^{m}\left(\prod_{j=i_{h}}^{i_{h+1}-1} W_{p_{j}}^{-}\left(f_{j+1}-f_{j}\right)\right) \rightarrow \prod_{h=1}^{m}\left(M \times \prod_{j=i_{h}}^{i_{h+1}-1} M\right)
$$

that is defined by

$$
\operatorname{EXP}\left(p, \ell, \prod_{h=1}^{m} \vec{p}_{h}\right)=\prod_{h=1}^{m}\left(\operatorname{Exp}_{h}(p, \ell), \iota\left(\vec{p}_{h}\right)\right)
$$

where $\vec{p}_{h}=\left(p_{i_{h}}, \cdots, p_{\left(i_{h+1}-1\right)}\right) \in \prod_{j=i_{h}}^{i_{h+1}-1} W_{p_{j}}^{-}\left(f_{j+1}-f_{j}\right)$ and $\iota: \prod_{j=i_{h}}^{i_{h+1}-1} W_{p-j}^{-} \rightarrow$ $\prod_{j=i_{h}}^{i_{h+1}-1} M$ is just the inclusion map. Now the following is easy to show

LEMmA 12.2. There exists a natural one to one correspondence

$$
\operatorname{EXP}^{-1}\left(\prod_{h=1}^{m} \Delta_{h}\right) \simeq \operatorname{Exp} p^{-1}\left(\prod_{h=1}^{m} \bigcap_{j=i_{h}}^{i_{h+1}-1} W_{p_{j}}^{-}\left(f_{j+1}-f_{j}\right)\right)
$$

where $\Delta_{h}$ is the diagonal in $M \times\left(\prod_{j=i_{h}}^{i_{h+1}-1} M\right)=M \times \cdots \times M$.

Now we are ready to formulate our transversality condition.

Definition 12.3. An element of $\mathcal{M}_{g}(M: \vec{f}, \vec{p}, \mathfrak{t})$ is called transversal if the map EXP above is transversal to $\prod_{h=1}^{m} \Delta_{h}$ at the point $\left(p, \ell, \prod_{j=i_{h}}^{i_{h+1}-1} \vec{p}_{h}\right)$ of $M \times$ $\operatorname{Gr}(\mathfrak{t}) \times\left(\prod_{j=i_{h}}^{i_{h+1}-1} W_{p_{j}}^{-}\left(f_{j+1}-f_{j}\right)\right)$ corresponding to the given element in $\mathcal{M}_{g}(M: \vec{f}, \vec{p}, \mathfrak{t})$ through the correspondences in Lemma 12.1 and 12.2. If this is the case at every point in $\mathcal{M}_{g}(M: \vec{f}, \vec{p}, \mathfrak{t})$, then we say that $\mathcal{M}_{g}(M: \vec{f}, \vec{p}, \mathfrak{t})$ is transversal.

For the convenience of the exposition, when the transversality in Definition 12.3 holds, we will often simply say that the map $\operatorname{Exp}: M \times G r(\mathfrak{t}) \rightarrow M^{m}$ is transversal to $\prod_{h=1}^{m} \bigcap_{j=i_{h}}^{i_{h+1}-1} W_{p_{j}}^{-}\left(f_{j+1}-f_{j}\right)$. One always has to appropriately interpret into that of $E X P$ whatever statement on the map Exp appears below.

The following lemma can be proven by a simple dimension counting argument using the transversality of the map EXP formulated in Definition 12.3.

Lemma 12.4. Suppose $\mathcal{M}(M: \vec{f}, \vec{p}, \mathfrak{t})$ is transversal in the sense of Definition 12.3. Then $\mathcal{M}(M: \vec{f}, \vec{p}, \mathfrak{t})$ is a smooth manifold of dimension

$$
\sum_{i=1}^{k} \mu\left(x_{i}\right)-(k-1) n+\operatorname{dim} G r(\mathfrak{t}) .
$$

We remark that it is not difficult to show

$$
\operatorname{dim} G r(\mathfrak{t})=k-3-\sum_{v \in C_{\text {int }}^{0}(T)}\left(k_{v}-3\right),
$$

where $n=\operatorname{dim} M$ and $k_{v}$ is the number of edges containing $v$. (See $\S 14$ for its proof.)

Now we prove the following transversality result.

Proposition 12.5. There exists a residual subset of $\left(C^{\infty}(M)\right)^{m}$ such that for $\vec{f}$ in it, every element of $\mathcal{M}_{g}(M: \vec{f}, \vec{p}, \mathfrak{t})$ is transversal in the sense of Definition 12.3.

Proof. Let $U_{i}$ be a sufficiently small neighborhood of $p_{i}$. We consider the subset of $\left(C^{\infty}(M)\right)_{0}^{m}$ consisting of the functions which coincides to the given $\vec{f}$ at $U_{i}$. We 
define

$$
\mathcal{M}_{g}(M: \vec{p}, \mathfrak{t})=\bigcup_{\vec{f} \in\left(C^{\infty}(M)\right)_{0}^{m}} \mathcal{M}_{g}(M: \vec{f}, \vec{p}, \mathfrak{t})
$$

We first remark that for $\vec{f}$ in a residual subset of $\left(C^{\infty}(M)\right)_{0}^{m}$ all $f_{j+1}-f_{j}$ are of the Morse-Smale type and so the unstable submanifolds of these functions are smooth. Therefore we take a such $\vec{f}_{0}$ and consider $\vec{f}$ 's only in a small neighborhood of $\vec{f}_{0}$.

The next step is to find a perturbation of $\vec{f}$ such that Exp become transversal to $\prod_{h=1}^{m} \cap_{j=i_{h}}^{i_{h+1}-1} W_{p_{j}}^{-}\left(f_{j+1}-f_{j}\right)$. We will choose the perturbation so that it does not change the unstable manifolds involved. For this purpose we choose open subsets $V_{i}$ of $M$ as follows. We write $v_{i} \prec v_{j}$ if the minimal path joining $v_{0}$ and $v_{j}$ contains $v_{i}$. Our open sets $V_{i} \subset M$ satisfies the following. Here we identify $T$ with its image by $I \in \mathcal{M}_{g}(M: \vec{f}, \vec{p}, \mathfrak{t})$.

(12.1.1) The intersection of $V_{i}$ with the minimal path joining $v_{i}$ and $v_{0}$ is nonempty. (12.1.2) If the intersection of $V_{i}$ with the minimal path joining $v_{j}$ and $v_{0}$ is non-empty then $v_{i} \prec v_{j}$.

(12.1.3) $V_{i}$ do not intersect the unstable manifolds $W_{p_{j}}^{-}\left(f_{j+1}-f_{j}\right), j=i_{h}, \cdots, i_{h+1}-1$ unless the dimension of $W_{p_{j}}^{-}\left(f_{j+1}-f_{j}\right)$ is $\operatorname{dim} M$.

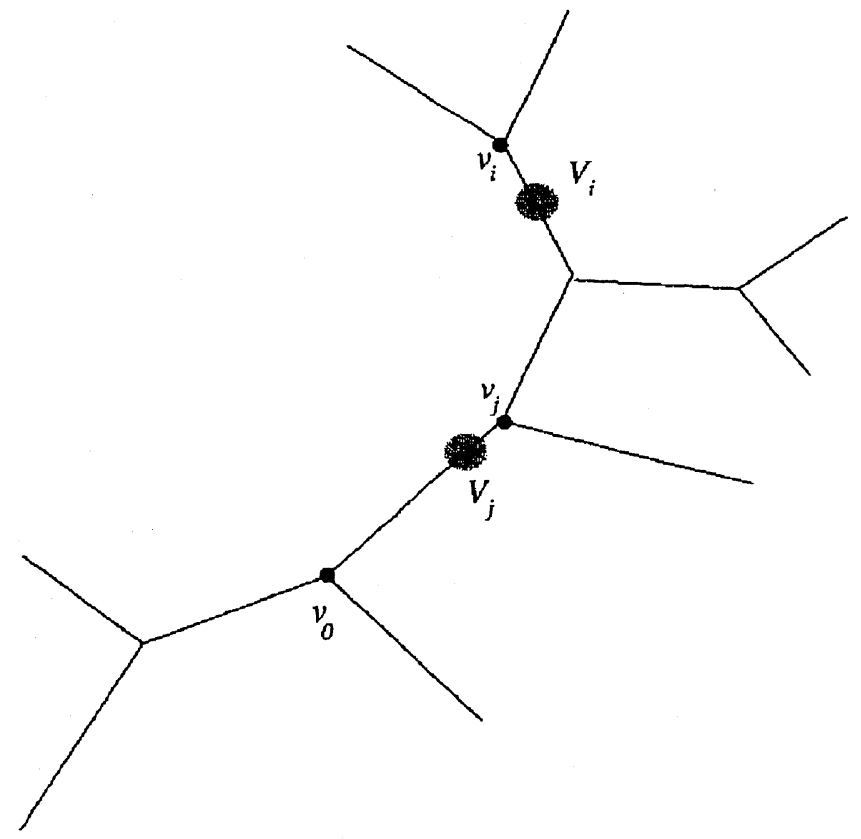

Figure 12.2

(It may happen that some $v_{i}$ coincides $v_{0}$. In that case we do not require (12.1.1) and just take $V_{i}$ empty.) One can find such open subsets by taking $V_{i}$ to be a sufficiently small neighborhood of the point $p$ which is on the path joining $v_{i}$ to $v_{0}$ and which is sufficiently close to $v_{i}$. 
Next we consider the map

$$
\widetilde{\operatorname{Exp}}: M \times G r_{k} \times \prod_{h=1}^{m} C_{0}^{\infty}\left(V_{i_{h}}\right) \rightarrow M^{m}
$$

such that $\widetilde{\operatorname{Exp}}(p, \ell, \vec{g})=\operatorname{Exp}\left(p, \ell, \vec{f}^{\prime}\right)$. Here the $i$ th component of $\vec{f}^{\prime}$ is $f_{i}+g_{h}$ where $i_{h} \leq i<i_{h+1}$. By abuse of notation we write $\vec{f}+\vec{g}$ for this element $\vec{f}^{\prime}$.

We consider

$$
\widetilde{\operatorname{Exp}}^{-1}\left(\prod_{h=1}^{m} \bigcap_{j=i_{h}}^{i_{h+1}-1} W_{p_{i}}^{-}\left(f_{j+1}-f_{j}\right)\right)
$$

Now we show :

LEMMA 12.6. $\widetilde{\operatorname{Exp}}^{-1}\left(\prod_{h=1}^{m} \cap_{j=i_{h}}^{i_{h+1}-1} W_{p_{i}}^{-}\left(f_{j+1}-f_{j}\right)\right)$ is a smooth submanifold of $M \times G r_{k} \times \prod_{h=1}^{m} C_{0}^{\infty}\left(V_{i_{h}}\right)$. Furthermore the restriction map of the projection $\pi$ : $M \times G r_{k} \times \prod_{h=1}^{m=1} C_{0}^{\infty}\left(V_{i_{h}}\right) \rightarrow \prod_{h=1}^{m} C_{0}^{\infty}\left(V_{i_{h}}\right)$

$$
\widetilde{\operatorname{Exp}}^{-1}\left(\prod_{h=1}^{m} \bigcap_{j=i_{h}}^{i_{h+1}-1} W_{p_{j}}^{-}\left(f_{j+1}-f_{j}\right)\right) \rightarrow \prod_{h=1}^{m} C_{0}^{\infty}\left(V_{i_{h}}\right)
$$

is a Fredholm map.

Once we have Lemma 12.6 then Proposition 12.5 is a consequence of the SardSmale transversality theorem [Sm]. (More precisely we replace $C_{0}^{\infty}\left(V_{i}\right)$ by a Banach space contained in it, as in Floer [Fl2].) Now we prove Lemma 12.6. It suffices to show that the map $\widetilde{E x p}$ is transversal to $\prod_{h=1}^{m} \cap_{j=i_{h}}^{i_{h+1}-1} W_{p_{j}}^{-}\left(f_{j+1}-f_{j}\right)$. (The Fredholmness is easy to show in the formulation of Lemma 12.4.) To prove it we are going to show that the differential of $\widetilde{E x p}$ is surjective. Let $(p, \ell, \vec{g}) \in M \times G r(\mathfrak{t}) \times \prod_{h=1}^{m} C_{0}^{\infty}\left(V_{i}\right)$, and $\left(q_{i}\right)=\widetilde{\operatorname{Exp}}(p, \ell, \vec{g})$. Let $X_{i} \in T_{q_{i}} M$. We are going to find $\vec{g}_{i}$ an $V \in T_{p} M$ such that

$$
\left.\frac{d \operatorname{Exp}\left(\exp _{p}(t V), \ell, \vec{f}+t \vec{g}\right)}{d t}\right|_{t=0}=\left(X_{1}, \cdots, X_{m}\right) .
$$

We find such a $g_{i}$ by induction of the order $\prec$ of $v_{i}$. We remark that Condition (12.1.2) implies that $g_{j}$ do not affect the $i$-th component of $\left.\frac{d E x p\left(e x p_{p}(t V), \ell, \vec{f}+t \vec{g}\right)}{d t}\right|_{t=0}$ unless $v_{i} \prec v_{j}$. Hence we can modify $g_{j}$ according to the order $\prec$ of $v_{i}$.

First suppose possibly there exists $v_{i}$ with $v_{0}=v_{i}$. Such an element is necessarily the smallest element among $v_{i}$ 's. Then clearly the $i$-th component of $\operatorname{Exp}(p, \ell, \vec{f})$ is $p$. Hence by putting $V=X_{i},(12.2)$ is satisfied for the $i$-th component.

Next we construct $g_{j}$. Assume we have already chosen $g_{i}$ for $i$ with $v_{i} \prec v_{j}$. Take $\vec{g}^{\prime}$ so that $g_{h}=0$ for those which are not yet chosen. We have already chosen $V$ also. Then denote the $j$-th component of $\left.\frac{d \operatorname{Exp}\left(\ell, \exp p_{p}(t V), \vec{f}+t \vec{g}^{\prime}\right)}{d t}\right|_{t=0}$ by $Y_{j}$. By Condition (12.1.1), we can find $g_{j}$ so that, if $\vec{g}_{j} \in \prod_{h=1}^{m} C_{0}^{\infty}\left(V_{i}\right)$ is the element whose $h$-th component is 0 unless $i=j$ and whose $j$-th component is $g_{j}$, then the $j$-th component of $\left.\frac{d \operatorname{Exp}\left(\exp _{p}(t V), \ell, \vec{f}+t \vec{g}_{j}\right)}{d t}\right|_{t=0}$ becomes $X_{j}-Y_{j}$. Then (for any choice of $g_{h}$ we make later) the $j$-th component of $\left.\frac{d E x p\left(\exp p_{p}(t V), \ell, \vec{f}+t \vec{g}\right)}{d t}\right|_{t=0}$ is $X_{j}$. Thus Lemma 12.6 now 
can be proved by induction. Hence the proof of Lemma 12.6 and so that of Proposition 12.5 .

13. Construction of the right inverse and the exact solutions. In this section, we use the transversality established in $\S 12$ and construct a right inverse of the linearized equation of the Cauchy Riemann equation at the approximate solutions constructed in Proposition 11.4. This construction is parallel to that in $\S 6,7$. So we will explain only the part where arguments are new. The main new point in the current situation appears when rephrasing the transversality condition as we did in the beginning of $\S 6$.

Let $(p, \ell) \in M \times G r_{k}$ be the element corresponding to the map $I: T \rightarrow M$ in $\mathcal{M}_{g}(M: \vec{f}, \vec{p}, \mathfrak{t})$ through Lemma 12.1. We assume that it is transversal in the sense of Definition 12.3.

We identify each interior edge $e$ of $T$ to $[0, \ell(e)]$ and exterior edge to $(-\infty, 0]$. Let $\chi_{e}:[0, \ell(e)] \rightarrow M$ or $\chi_{e}:(-\infty, 0] \rightarrow M$ be the restriction of $I$. We put

$$
W_{I}^{1, p}=\left\{\left(c_{e}\right) \in \prod_{e \in C^{1}(T)} W^{1, p}\left(\chi_{e}^{*} T M\right) \mid c_{e}(v)=c_{e^{\prime}}(v) \text { if } v \in e, v \in e^{\prime}, v \in C_{i n t}^{0}(T)\right\}
$$

There is a map :

$$
L_{I}: W_{I}^{1, p} \rightarrow \prod_{e} L^{p}\left(\chi_{e}^{*} T M\right) .
$$

whose $e$-component is given by

$$
L_{e}=L_{\chi_{e}}:=\nabla_{\dot{\tau}}+\nabla \operatorname{grad}\left(f_{l e f(e)}-f_{r i g(e)}\right)
$$

We have :

LEMMA 13.1. $L_{I}$ is surjective when $I \in \mathcal{M}_{g}(M: \vec{f}, \vec{p}, \mathfrak{t})$ is transversal in the sense of Definition 12.2.

Proof. We consider the space :

$$
W_{I, i n t}^{1, p}=\left\{\left(c_{e}\right) \in \prod_{e \in C_{i n t}^{1}(T)} W^{1, p}\left(\chi_{e}^{*} T M\right) \mid c_{e}(v)=c_{e^{\prime}}(v) \quad \text { if } v \in e \cap e^{\prime}, v \in C_{i n t}^{0}(T)\right\}
$$

We define the map

$$
L_{I}^{i n t}: W_{I, i n t}^{1, p} \rightarrow \prod_{e \in C_{\text {int }}^{1}(T)} L^{p}\left(\chi_{e}^{*} T M\right)
$$

in a similar way. We also define the evaluation map

$$
e v: W_{I, \text { int }}^{1, p} \rightarrow \underset{h=1}{\oplus} T_{I\left(v_{i_{h}}\right)}(M)
$$

by

$$
\left(c_{e}\right) \mapsto\left(c_{e_{h}^{\prime}}\left(v_{i_{h}}\right)\right) .
$$

Here $e_{h}^{\prime}$ is any interior edge containing $v_{i_{h}}$. Then the transversality condition in Definition 12.3 for $(\ell, p)$ is equivalent to the surjectivity of the composition of the map

$$
\operatorname{Ker} L_{I}^{i n t} \stackrel{e v}{\longrightarrow} \oplus_{h=1}^{m} T_{I\left(v_{i_{h}}\right)}(M) \stackrel{\text { proj }}{\longrightarrow} \oplus_{h=1}^{m} N_{I\left(v_{i_{h}}\right)}\left(\bigcap_{j=i_{h}}^{i_{h+1}-1} W_{p_{h}}\left(f_{j+1}-f_{j}\right)\right)
$$


Here $N_{I\left(v_{i_{h}}\right)}(\cdot)$ means the normal bundle of $(\cdot)$. Again the precise formulation of this statement should follow the kinds of Lemma 12.2 and Definition 12.3 in their linearized version. We leave this obvious translation to readers.

Now we take an arbitrary element $\left(b_{e}\right) \in \prod L^{p}\left(\chi_{e}^{*} T M\right)$. First let $e \in C_{\text {int }}^{1}(T)$, $v$ be one of the vertex of $e$ and $V \in T_{I(v)} M$. By the existence of solution of Cauchy problem for ordinary differential equation, we can find $c_{e}(V) \in W^{1, p}\left(\chi_{e}^{*} T M\right)$ such that

$$
\begin{aligned}
L_{e}\left(c_{e}(V)\right) & =b_{e} \\
c_{e}(V)(v) & =V
\end{aligned}
$$

Now we use this fact and the fact that $T$ is a tree to find $\left(c_{e}^{\prime}\right) \in W_{I, \text { int }}^{1, p}$ such that

$$
L_{I}^{i n t}\left(\left(c_{e}^{\prime}\right)\right)=\left(b_{e}\right)
$$

Next we consider an exterior edge $e_{i}$. (We are again using the same notations as in the beginning of $\S 12$.) Then, for each $W_{i} \in T_{I\left(v_{i}\right)}\left(W_{p_{i}}^{-}\left(f_{i+1}-f_{i}\right)\right)$, we have

$$
c\left(e_{i}, W_{i}\right) \in W^{1, p}\left(\chi_{e_{i}}^{*} T M\right)
$$

such that

$$
\begin{aligned}
L_{e_{i}}\left(c\left(e_{i}, W_{i}\right)\right) & =b\left(e_{i}\right) \\
c\left(e_{i}, W_{i}\right)\left(v_{i}\right) & =W_{i}
\end{aligned}
$$

Now we consider $c_{e}^{\prime}\left(v_{i_{h}}\right) \in T_{I\left(v_{i_{h}}\right)} M$. Again the transversality in Definition 12.3 implies in particular that we can find

$$
V_{h} \in N_{I\left(v_{i_{h}}\right)}\left(\bigcap_{j=i_{h}}^{i_{h+1}-1} W_{p_{h}}\left(f_{j+1}-f_{j}\right)\right)
$$

and

$$
W_{h} \in T_{I\left(v_{i_{h}}\right)}\left(\bigcap_{j=i_{h}}^{i_{h+1}-1}\left(W_{p_{h}}^{-}\left(f_{j+1}-j_{j}\right)\right)\right)
$$

such that

$$
W_{h}-V_{h}+c_{e}^{\prime}\left(v_{i_{h}}\right)=0 .
$$

Using the surjectivity of (13.1) we find $\left(d_{e}\right) \in \operatorname{Ker} L_{I}^{\text {int }}$ such that $e v\left(d_{e}\right)=\left(W_{h}\right)$. Now we put

$$
\begin{aligned}
c_{e}=c_{e}^{\prime}+d_{e} & \text { if } e \in C_{i n t}^{1}(T) \\
c_{e_{j}}=c\left(e_{j}, W_{h}\right) & \text { if } e_{j} \in C_{e x t}^{1}(T), i_{h} \leq j<i_{h+1}
\end{aligned}
$$

By (13.2)-(13.4), we have

$$
c_{e}\left(v_{j}\right)=c_{e_{h}}\left(v_{j}\right) \quad j=i_{h}, \cdots, i_{h+1}-1
$$

Hence $c \in W_{I}^{1, p}$. By definition $L_{I}\left(c_{e}\right)=\left(b_{e}\right)$, as required.

Once we establish Lemma 13.1, we can imitate the arguments in the proofs of Propositions 6.1 and 7.1 to obtain a right inverse of the linearization of $\bar{\partial}_{J}$ at the elements defined in Proposition 11.7. Then using the estimates in Proposition 11.4 together with the above discussion on the right inverse, one can repeat the same proof, with obvious modifications, that was carried out for the case $k=3$ in $\S 7-9$. Hence 
we have constructed the required diffeomorphism in a neighborhood of each stratum $G r(\mathfrak{t})$.

Proposition 13.2. For sufficiently small $\epsilon>0$, there exists a constant $C$ and a smooth map

$$
\Xi_{\mathfrak{t}: \alpha}^{\epsilon}: U(\mathfrak{t}: \epsilon, \alpha) \times_{\pi_{\epsilon}} \mathcal{M}_{g}(M, \vec{f}, \vec{p}) \rightarrow \mathcal{M}_{J}\left(T^{*} M: \vec{\Lambda}^{\epsilon}, \vec{x}^{\epsilon}\right) \subset \operatorname{Map}\left(T^{*} M: \vec{\Lambda}^{\epsilon}, \vec{x}^{\epsilon}\right)
$$

such that

(13.7.1) Figure 11.2 commutes and

$$
\left|\Xi_{\mathrm{t}: \alpha}^{\epsilon}-\Psi_{\mathrm{t}: \alpha}^{\epsilon}\right| \leq C \epsilon^{\frac{2+(p-1) \alpha}{p}}
$$

In the next remaining sections, we will patch these diffeomorphism together and obtain the global diffeomorphism asked in the Main Theorem, Theorem 1.7.

14. Gluing moduli spaces (Metric ribbon tress). In this section, we reprove Stasheff's theorem and also we make clearer the relation between moduli spaces of graphs and genus zero open Riemann surfaces.

THEOREM 14.1. [Compare with (Stasheff [St1])] $G r_{k}$ can be given a smooth structure with respect to which it becomes diffeomorphic to $\mathbb{R}^{k-3}$.

We here first recall a gluing construction of graph modulies. Let $\mathfrak{t}=(T, i, p) \in G r_{k}$ and $k_{v} ; v \in C_{\text {int }}^{0}(T)$ be the number of edges containing $v$. We use the map :

$$
\Phi_{\mathfrak{t}}: G r(\mathfrak{t}) \times \prod_{v \in C_{i n t}^{0}(\mathfrak{t})} G r_{k_{v}} \rightarrow G r_{k}
$$

The construction is by gluing and is similar to the proof of Lemma 11.3. More precisely, let

$$
\left(\ell,\left(\ell_{v}\right)\right) \in G r(\mathfrak{t}) \times \prod_{v \in C_{i n t}^{0}(\mathfrak{t})} G r_{k_{v}}
$$

Here $\ell_{v} \in G r\left(\mathfrak{c}_{v}\right) \subset G r_{k_{v}}$ and $\mathfrak{c}_{v}=\left(C_{v}, i_{v}, p_{v}\right)$. We replace the vertex $v \in T$ by the tree $C_{v}$. Namely we identify $k_{v}$ edges containing $v$ and $k_{v}$ exterior edges of $C_{v}$, using their orders. Then we obtain a graph. Together with its ribbon structure and order we denote it by $\widehat{\mathfrak{t}}$. Then $\Phi_{\mathfrak{t}}\left(\ell,\left(\ell_{v}\right)\right)=\ell^{\prime} \in G r(\mathfrak{t})$. Here $\ell^{\prime}(e)=\ell(e)$ if $e \in C_{i n t}^{1}(T)$ and $\ell^{\prime}(e)=\ell_{v}(e)$ if $e \in C_{\text {int }}^{1}\left(C_{v}\right)$.

Let $\mathfrak{c}_{v} \in G_{k_{v}}$ and $\mathfrak{c}_{u, v} \in G_{k_{u, v}}$, where $u \in C_{\text {int }}^{0}\left(\mathfrak{c}_{v}\right)$ and $k_{u, v}$ be the number of edges containing $u$. We then have the following commutative diagram :

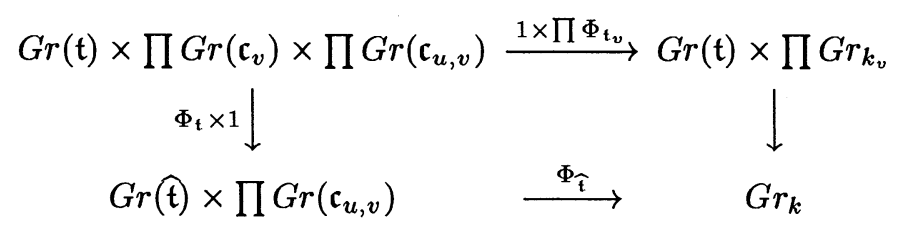

Figure 14.1

Now we start with the proof of Theorem 14.1. We prove this by induction on $k$. Suppose that the theorem holds for $k^{\prime}<k$. Let $\mathfrak{t}_{k} \in G r_{k}$ be the graph which has no interior edge. Then $G r\left(\mathfrak{t}_{k}\right)$ is a point. We first prove that $G r_{k}-G r\left(\mathfrak{t}_{k}\right)$ is a topological manifold. Let $\mathfrak{t} \neq \mathfrak{t}_{k}$. Then $k_{v}<k$ for each $v \in C_{i n t}^{0}(\mathfrak{t})$. Hence, by the induction hypothesis, $G r_{k_{v}}$ is homeomorphic to $\mathbb{R}^{k_{v}-3}$. Using it we find that $\operatorname{Gr}(\mathfrak{t}) \times \prod G r\left(k_{v}\right)$ 
is homeomorphic to $\mathbb{R}^{k-3}$. (Note that $G r(\mathfrak{t})$ is a cell.) Therefore

$$
\Phi_{\mathfrak{t}}: G r(\mathfrak{t}) \times \prod G r_{k_{v}} \rightarrow G r_{k}
$$

is a homeomorphism from $\mathbb{R}^{k-3}$ to a neighborhood of $G r(\mathfrak{t})$. We regard them as a coordinate chart. So this gives a structure of topological manifold to $G r_{k}-G r\left(t_{k}\right)$.

We next prove that these charts give a smooth structure to $G r_{k}-G r\left(\mathfrak{t}_{k}\right)$. The proof of this fact is again by induction. From the induction hypothesis, there exists a smooth structure on $G r_{k^{\prime}}$ with respect to which it is diffeomorphic to $\mathbb{R}^{k^{\prime}-3}$ for $k^{\prime}<k$. Then the $C^{\infty}$ compatibility of the above charts is a consequence of the commutativity of Figure 14.1 and the construction of the diffeomorphism $G r_{k_{v}} \simeq \mathbb{R}^{k_{v}-3}$, which we are going to explain later in this section.

We have thus obtained a smooth structure on $G r_{k}-G r\left(\mathfrak{t}_{k}\right)$. We remark that there is an action of $\mathbb{R}_{+}$on $G r_{k}-G r\left(\mathfrak{t}_{k}\right)$. This action is free and $G r_{k}$ is the cone of the quotient space of this action. Hence to complete the proof of our theorem, we have only to prove that the quotient space is diffeomorphic to the sphere. We will prove this fact by using a relationship of $G r_{k}$ to the other moduli space $\mathfrak{T}_{0, k}$.

Our basic observation to prove Theorem 14.1 is that the natural cell decompositions of the compactifications of $G r_{k}$ and $\mathfrak{T}_{0, k}$ is the dual decomposition to each other. We now explain this point more precisely.

Let $\overline{\mathfrak{T}}_{0, k}$ be the compactification of $\mathfrak{T}_{0, k}$ as we explained in $\S 10$. It has a cell decomposition such that each cell corresponds to an element of $G_{k}$ as follows. We define cells $\Delta(\mathfrak{t})$ for each $\mathfrak{t} \in G_{k}$. An element of $\overline{\mathfrak{T}}_{0, k}$ is identified to $\left[\Sigma ; z_{1}, \cdots, z_{k} ; \tau\right]$ such that $\Sigma$ is a genus zero stable curve $z_{i}$ are regular points of $\Sigma$ and $\tau$ is an anti holomorphic involution of $\Sigma$ such that $\tau\left(z_{i}\right)=z_{i}$. If $\left[\Sigma ; z_{1}, \cdots, z_{k} ; \tau\right]$ is in the interior $\mathfrak{T}_{0, k}$ of our moduli space, namely if $\Sigma$ is nonsingular, we say that $\left[\Sigma ; z_{1}, \cdots, z_{k} ; \tau\right] \in$ $\Delta\left(\mathfrak{t}_{k}\right)$. (Here $\mathfrak{t}_{k}$ is the graph without an interior edge.) Otherwise we obtain a ribbon graph $\mathfrak{t}=(T, i, p)$ as we explained in $\S 10$. We say $\left[\Sigma ; z_{1}, \cdots, z_{k} ; \tau\right] \in \Delta(\mathfrak{t})$. It is easy to prove that $\Delta(\mathfrak{t})$ is a cell. It also follows that their dimensions are given by

$$
\operatorname{dim} \Delta(\mathfrak{t})=\sum_{v \in C_{i n t}^{0}(\mathfrak{t})}\left(k_{v}-3\right)
$$

where $k_{v}$ is the number of edges containing $v$. These cells will turn out to be the dual cells of $G r(t)$ as we will prove later in this section. We now calculate the dimension of $G r(\mathfrak{t})$. We first remark that

$$
\operatorname{dim} G r(\mathfrak{t})=\sharp C_{i n t}^{1}(\mathfrak{t})
$$

On the other hand, by Euler's formula using the fact that our graph is a connected tree, we have

$$
1=\sharp C^{0}(\mathfrak{t})-\sharp C^{1}(\mathfrak{t})
$$

We also have $\sharp C_{e x t}^{1}(\mathfrak{t})=\sharp C_{e x t}^{0}(\mathfrak{t})=k$, and

$$
\begin{aligned}
2 \sharp C^{1}(\mathfrak{t}) & =k+\sum_{v \in C_{i n t}^{0}(\mathfrak{t})} k_{v} \text { and so } \\
2 \sharp C_{i n t}^{1}(\mathfrak{t}) & =-k+\sum_{v \in C_{i n t}^{0}(\mathfrak{t})} k_{v} .
\end{aligned}
$$


Combining these, we have

$$
\begin{aligned}
3 & =3 \sharp C_{\text {int }}^{0}(\mathfrak{t})-3 \sharp C_{\text {int }}^{1}(\mathfrak{t}) \\
& =3 \sum_{v \in C_{i n t}^{0}(\mathfrak{t})} 1-2 \sharp C_{i n t}^{1}(\mathfrak{t})-\sharp C_{i n t}^{1}(\mathfrak{t}) \\
& =-\sum_{v \in C_{\text {int }}^{0}(\mathrm{t})}\left(k_{v}-3\right)+k-\sharp C_{\text {int }}^{1}(\mathfrak{t}) .
\end{aligned}
$$

Hence from (14.1) and (14.2), we conclude

$$
\operatorname{dim} G r(\mathfrak{t})=k-3-\sum_{v \in C_{i n t}^{0}(\mathfrak{t})}\left(k_{v}-3\right)=\operatorname{dim} \mathfrak{T}_{0, k}-\operatorname{dim} \Delta(\mathfrak{t}) .
$$

Next we recall the definition of the dual cell decomposition. First let $X$ be a smooth manifold and $X_{a}, a \in I, I$ being a some indexing set, be smooth submanifolds such that their closures $\overline{X_{a}}$ become smooth submanifolds with corners. We say that they consist of smooth cell decomposition if the followings are satisfied :

(14.5.1) $X_{a}$ are disjoint from one another and $\coprod_{a \in I} X_{a}=X$.

(14.5.2) $X_{a}$ is diffeomorphic to $\mathbb{R}^{|a|}$, and $\overline{X_{a}}$ (after smoothing out their corners) is diffeomorphic to $D^{|a|}$. Here $|a|$ is a positive integer.

(14.5.3) The boundary $\partial D^{|a|}$ of $D^{|a|}$ is a union of some of the $X_{b}$ 's with $|b|<|a|$.

Given such a decomposition we define its dual decomposition as follows. Our definition is given by induction on the dimension $n$ of $X$. Suppose that the dual decomposition is defined for manifolds of dimension $<n$. Let $a \in I$ and $p \in X_{a}$. We consider the normal bundle $N_{p} X_{a}$ and its unit sphere bundle $S N_{p} X_{a}$. For each $X_{b}$ with $\overline{X_{b}} \supset X_{a}$ we consider the intersection $S N_{p} X_{a} \cap T_{p} \overline{X_{b}}$ which is a cell. These cells define a cell decomposition of $S N_{p} X_{a}=S^{n-|a|-1}$. Therefore by the induction hypothesis we obtain a dual decomposition of it. We add one more cell of dimension $n-|a|$ to the dual decomposition of $S N_{p} X_{a}=S^{n-|a|-1}$. The we get a cell decomposition of $D^{n-|a|}$ which we define to be $\overline{Y_{a}}$.

We now glue these cells as follows. For $a, b \in I$ we are going to construct an inclusion $\overline{Y_{a}} \subset \overline{Y_{b}}$. We construct it in case $|a|=|b|+1$. (In the general case we can construct the inclusion by composing inclusions of this case.) Let $q \in X_{b}$. We choose a unit normal vector $v \in S N_{q} X_{b}$ which are tangent to $\overline{X_{a}}$ and $\exp (\epsilon v)$ is contained in $X_{a}$ for small $\epsilon$. Since $|a|=|b|+1$ there is only one such vector. We consider the decomposition of $S N_{q} X_{b}$ induced by the decomposition of $X$. This decomposition induces that of $S N_{v}\left(S N_{q} X_{b}\right)$. One finds easily that the decomposition obtained on $S N_{v}\left(S N_{q} X_{b}\right)$ is the same as the decomposition of $S N_{p} X_{a}$. Hence by construction, the cell of the dual decomposition of $S N_{q} X_{b}$ which corresponds to the point $v \in S N_{q} X_{b}$ is isomorphic to $\overline{Y_{a}}$. We thus obtain an inclusion $\overline{Y_{a}} \subset \overline{Y_{b}}$.

By gluing these cells that we have defined as above $\overline{Y_{a}}$ using the inclusion, we obtain a cell complex $Y$. It is easy to see that it has a smooth structure such that $Y_{a}$ gives its smooth cell decomposition. Then we have

LeMma 14.2. $X$ is diffeomorphic to $Y$.

Proof. The barycentric subdivision of $X$ becomes also a subdivision of $Y$.

Now we can state Theorem 14.1 more precisely in our context.

THEOREM 14.3. Let us compactify $G r_{k}$ using the $\mathbb{R}_{+}$action on $G r_{k}-G r\left(\mathfrak{t}_{k}\right)$ and denote by $\overline{G r}_{k}$ the compactification. Then $\left(\overline{G r}_{k},\{\overline{G r(\mathfrak{t})\}})\right.$ is the dual cell decomposition 
to $\left(\overline{\mathfrak{T}}_{0, k},\{\mathfrak{T}(\mathfrak{t})\}\right)$.

Proof. The proof is again by induction on $k$. Suppose that Theorem 14.3 is true for $k^{\prime}<k$. We consider the cell $G r(\mathfrak{t})$ and take a point $p$ on it. Then $N_{p} G r(\mathfrak{t})$ together with its induced decomposition can be identified with $\prod_{v \in C_{i n t}^{0}(\mathfrak{t})} G r_{\left(k_{v}-3\right)}$. We assume that $t \neq t_{k}$. Then using the dimension formula (14.4) and the induction hypothesis, we derive that $S N_{p} G r(\mathfrak{t})$ is the dual decomposition of the boundary

$$
\partial \prod_{v \in C_{i n t}^{0}(\mathfrak{t})} \overline{\mathfrak{T}}_{\left(k_{v}-3\right)}
$$

We put $X=G r_{k}$ and use the notation in the definition of the dual decomposition. Then we find

$$
\overline{Y_{\mathfrak{t}}}=\prod_{v \in C_{i n t}^{0}(\mathfrak{t})} \overline{\mathfrak{T}}_{\left(k_{v}-3\right)}
$$

Since this holds for arbitrary $\mathfrak{t}$ except possibly $\mathfrak{t}_{k}$ we find that $\partial \overline{G r}_{k}$ and $\partial \overline{\mathfrak{T}}_{(k-3)}$ are dual to each other. It follows that $\partial \overline{G r}_{k} \simeq S^{k-4}$. Thus as we already explained $G r_{k}$ is a smooth manifold. We then conclude from definition that $\left(\overline{G r}_{k},\{\overline{G r(t)}\}\right)$ is the dual cell decomposition to $\left(\overline{\mathfrak{T}}_{0, k},\{\mathfrak{T}(\mathfrak{t})\}\right)$. The proof of Theorem 14.3 and so that of Theorem 14.1 is now complete.

Now we are in the position to prove Theorem 10.4.

We first remark that by construction we can extend the map $\Theta: G r_{k} \rightarrow \mathfrak{T}_{0, k}$ to its compactification $\bar{\Theta}: \overline{G r_{k}} \rightarrow \overline{\mathfrak{T}}_{0, k}$. Here $\partial G r_{k}=\overline{G r}_{k}-G r_{k}$ consists of $(\mathfrak{t}, \ell)$ such that $\ell(e)=\infty$ for some interior edge $e$.

We first prove that $\Theta$ is surjective by induction on $k$. We will prove that it is of degree one (namely induces an isomorphism $\bar{\Theta}^{*}: H^{k-3}\left(\overline{\mathfrak{T}}_{0, k}, \partial \overline{\mathfrak{T}}_{0, k} ; \mathbb{Z}\right) \rightarrow$ $\left.H^{k-3}\left(\overline{G r}_{k}, \partial \overline{G r}_{k} ; \mathbb{Z}\right)\right)$ at the same time. When $k=3$ then $G r_{3}=\mathfrak{T}_{0,3}=$ one point. Hence there is nothing to show. Suppose that Lemma 10.3 is true for $k-1$. Then, we find that the restriction of $\bar{\Theta}: \overline{G r}_{k} \rightarrow \overline{\mathfrak{T}}_{0, k}$ to each cell in $\partial \overline{G r}_{k}$ is surjective and is of degree one to the corresponding dual cell in $\partial \overline{\mathfrak{T}}_{0, k}$. Hence the restriction of $\bar{\Theta}$ to $\partial \overline{G r}_{k}$ is surjective to $\partial \overline{\mathfrak{T}}_{0, k}$ and is of degree one. Since we already proved that $G r_{k} \simeq \mathfrak{T}_{0, k} \simeq \mathbb{R}^{k-3}$, it follows that $\Theta: G r_{k} \rightarrow \mathfrak{T}_{0, k}$ is surjective and of degree one. The proof of the surjectivity of $\Theta$ is complete now.

We next prove that $\Theta$ is a diffeomorphism. We remark that it suffices to show that $\Theta$ is a local diffeomorphism. This is because our map is between two spaces homeomorphic to the disk and is of degree one.

We first consider the top-stratum i.e., the case in which $t \in G_{k}$ is a trivalent graph.

LEMmA 14.4. Let $t \in G r_{k}$ be a trivalent graph. Then the differential of $\Theta$ is invertible at points of $G r(\mathfrak{t})$.

Proof. Let $\ell \in G r(t)$. We consider $\Theta(\ell)$. We take its double $\widehat{\Theta}(\ell)$. It is a Riemann surface of genus 0 with $k$ ends. We also have an explicit diffeomorphism between each of the ends of $\widehat{\Theta}(\ell)$ and $(-\infty, 0] \times S^{1}$. Moreover $\widehat{\Theta}(\ell)$ has an anti-holomorphic involution $\tau$.

By the general theory of deformation of complex structures, the compactly supported Dolbeault cohomology

$$
H_{c p t}^{0,1}(\widehat{\Theta}(\ell), \mathfrak{O}(T \widehat{\Theta}(\ell)))
$$

of the tangent bundle is canonically isomorphic to and so can be identified with the 


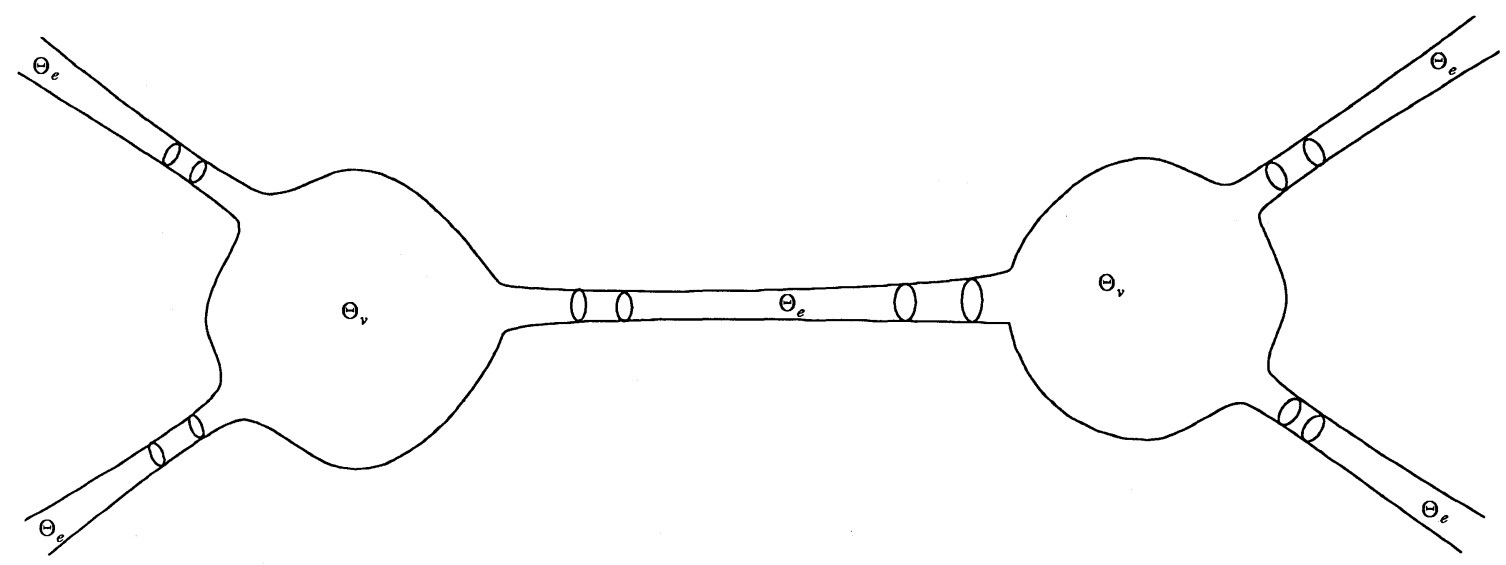

FIGURE 14.2

tangent space of the moduli space, $T_{\widehat{\Theta}(\ell)} \mathfrak{T}_{0, k}^{\mathbb{C}} \simeq \mathbb{C}^{k-3}$. Moreover $\tau$ induces an antiholomorphic involution of the Dolbeault cohomology and so its fixed point set corresponds to $T_{\Theta(\ell)} \mathfrak{T}_{0, k} \simeq \mathbb{R}^{k-3}$ under the identification.

By construction we have $k-3$ annuli $\widehat{\Theta}_{e} \simeq[\epsilon, \ell(e)-\epsilon] \times S^{1}$ embedded in $\widehat{\Theta}(\ell)$, which corresponds each of the element $e$ of $C_{i n t}^{1}(\mathfrak{t})$. We have a homomorphism induced by inclusion ;

$$
\bigoplus_{e} H_{c p t}^{0,1}\left(\widehat{\Theta}_{e}, \mathfrak{O}\left(T \widehat{\Theta}_{e}\right)\right) \rightarrow H_{c p t}^{0,1}(\widehat{\Theta}(\ell), \mathfrak{O}(T \widehat{\Theta}(\ell)))
$$

Using the fact that $\widehat{\Theta}(\ell)-\cup \widehat{\Theta}_{e}$ is a union of $\mathbb{C} P^{1}-\{3$ points $\}$, whose deformation is trivial, we find that (14.5) is an isomorphism.

On the other hand, the real part of $H_{c p t}^{0,1}\left(\widehat{\Theta}_{e}, \mathfrak{O}\left(T \widehat{\Theta}_{e}\right)\right)$ (the fixed point set of $\tau$ ) is $\mathbb{R}$. This group is identified with the real part of the tangent space of the moduli of complex structure on annulus. Thus the differential of $\Theta$ is identified to the real part of the map (14.5) and hence is invertible.

By now we have proved that $\Theta$ is locally a diffeomorphism at each top dimensional strata. We now prove by induction on $k$ that it is a local diffeomorphism everywhere.

If $k=3$, there is nothing to show. We assume that it is proved for $k-1$ and smaller. We are going to prove that $\Theta$ is locally a diffeomorphism for $k$ on $G r_{k}-G r\left(t_{k}\right)$.

Let $\mathfrak{t} \neq \mathfrak{t}_{k}$ and $\ell \in G r(\mathfrak{t})$. We have $\Theta_{e} \subset \Theta(\ell), \Theta_{v} \subset \Theta(\ell)$, for each $e \in C_{\text {int }}^{1}(\mathfrak{t})$ and $v \in C_{i n t}^{0}(\mathfrak{t})$ such that they are disjoint to each other, $\Theta_{e}$ is a rectangle, and $\Theta_{v}$ is obtained by gluing $k_{v}$ rectangles in a way similar to the definition of $\Theta$. Let $\widehat{\Theta}_{e}, \widehat{\Theta}_{v}$ be their double (see Figure 14.2).

LEMMA 14.5. The map

$$
\bigoplus_{e} H_{c p t}^{0,1}\left(\widehat{\Theta}_{e}, \mathfrak{O}\left(T \widehat{\Theta}_{e}\right)\right) \oplus \bigoplus_{v} H_{c p t}^{0,1}\left(\widehat{\Theta}_{v}, \mathfrak{O}\left(T \widehat{\Theta}_{v}\right)\right) \rightarrow H_{c p t}^{0,1}(\widehat{\Theta}(\ell), \mathfrak{O}(T \widehat{\Theta}(\ell)))
$$

is an isomorphism.

Proof. We can find $\Theta_{i} \simeq\left[0, C_{i}\right] \times[0,1] \subset \widehat{\Theta}(\ell), i=1, \cdots, 2 \sharp C_{i n t}^{1}(\mathfrak{t})$ and $\Theta_{i} \simeq$ $(-\infty, 0] \times[0,1] \subset \widehat{\Theta}(\ell), i=2 \sharp C_{i n t}^{1}(\mathfrak{t})+1, \cdots 2 \sharp C_{i n t}^{1}(\mathfrak{t})+\sharp C_{e x t}^{1}(\mathfrak{t})$, such that $\widehat{\Theta}(\ell)-\cup \widehat{\Theta}_{i}$ is a compact subset of $\cup \widehat{\Theta}_{e} \cup \cup \widehat{\Theta}_{v}$. We remark that

$$
H^{0,1}\left(\widehat{\Theta}_{i}, \mathfrak{O}\left(T \widehat{\Theta}_{i}\right)\right)=0 .
$$


(The cohomology here is not one with compact support.) Now let $u \in H_{c p t}^{0,1}(\widehat{\Theta}(\ell)$, $\mathfrak{O}(T \widehat{\Theta}(\ell))$ i.e., $\Gamma\left(\Lambda^{0,1} \otimes T \widehat{\Theta}(\ell)\right)$ with $\bar{\partial} u=0, \tau u=u$. We find $w_{i} \in \Gamma(T \widehat{\Theta}(\ell))$ such that $\bar{\partial} w_{i}=u, \tau w_{i}=w_{i}$. We take $u^{\prime}=u-\sum \bar{\partial}\left(\chi_{i} w_{i}\right)$. Here $\{\chi\}$ is an appropriate partitions of unity. Then we have $[u]=\left[u^{\prime}\right]$, and $u^{\prime}$ is supported in a compact subset of $\cup \widehat{\Theta}_{e} \cup \cup \widehat{\Theta}_{v}$. This proves that the homomorphism in Lemma 14.5 is surjective. Then it follows by dimension counting that it is an isomorphism.

Now we remark that

$$
T_{\ell} G r_{k}=\bigoplus_{e \in C_{i n t}^{1}(t)} \mathbb{R} \oplus \bigoplus_{v \in C_{i n t}^{0}(t)} T_{0} G r_{k_{v}}
$$

Here $0 \in G r_{k_{v}}$ is the unique point in $G r\left(t_{k_{v}}\right)$, where $\mathfrak{t}_{k_{v}}$ is the graph without interior edges. The differential of $\Theta$ at $\ell$ has the component corresponding to $e$ which is identified with the restriction of the isomorphism in Lemma 14.5 to the real point of $H_{c p t}^{0,1}\left(\widehat{\Theta}_{e}, \mathfrak{O}\left(T \widehat{\Theta}_{e}\right)\right)$.

On the other hand by induction hypothesis, we see that the map

$$
T_{0} G r_{k_{v}} \rightarrow H_{c p t}^{0,1}\left(\widehat{\Theta}_{v}, \mathfrak{O}\left(T \widehat{\Theta}_{v}\right)\right)
$$

induced by the differential of $\Theta$ is an isomorphism to the real point. Therefore, by Lemma 14.5, the differential of $\Theta$ is an isomorphism at $\ell \in G r(\mathfrak{t})$ when $t \neq t_{k}$.

Now using the facts that both $G r_{k}$ and $\mathfrak{T}_{0, k}$ is homeomorphic to $\mathbb{R}^{k-3}$ and that $\Theta$ is a diffeomorphism at boundary (which follows from the induction hypothesis), we can prove that $\Theta$ is a homeomorphism. We can then choose a differentiable structure of $G r_{k}$ at $G r\left(t_{k}\right)$ such that $\Theta$ is a diffeomorphism at $G r\left(t_{k}\right)$ also. The proof of Theorem 10.4 is now complete.

15. Gluing moduli spaces (Graph flows). Using the result of the previous sections, we are going to prove Theorem 1.6. Namely we construct a smooth structure on the moduli space $\mathcal{M}_{g}(M: \vec{f}, \vec{p})=\cup_{\mathfrak{t}} \mathcal{M}_{g}(M: \vec{f}, \vec{p}, \mathfrak{t})$. We have already proved in Lemma 12.4 that for each $\mathfrak{t} \in G r_{k}$ and generic $\vec{f}$, the moduli space $\mathcal{M}_{g}(M: \vec{f}, \vec{p}, \mathfrak{t})$ is a smooth manifold of dimension

$$
\operatorname{dim} \mathcal{M}_{g}(M: \vec{f}, \vec{p}, \mathfrak{t})=n-3-\sum_{v \in C_{\text {int }}^{0}(\mathfrak{t})}\left(k_{v}-3\right)+\sum \mu\left(x_{i}\right)-(k-1) n .
$$

Our task in this section is to glue them together to obtain a smooth manifold $\mathcal{M}_{g}(M$ : $\vec{f}, \vec{p})$.

We first recall the definition of

$$
G r(\mathfrak{t})=\left\{\ell: C_{\text {int }}^{1}(\mathfrak{t}) \rightarrow \mathbb{R} \mid \ell(e)>0\right\} .
$$

We put

$$
G r^{+}(\mathfrak{t})=\left\{\ell: C_{\text {int }}^{1}(\mathfrak{t}) \rightarrow \mathbb{R} \mid \ell(e) \geq 0\right\}
$$

We then find that

$$
G r^{+}(\mathfrak{t})=\coprod_{\mathfrak{t}^{\prime}: \mathfrak{t} \succ \mathfrak{t}^{\prime}} G r\left(\mathfrak{t}^{\prime}\right)
$$

We recall here that $t \succ t^{\prime}$ means that $t^{\prime}$ is obtained by shrinking some of the interior edges of $t$. Note that the transversality is also satisfied for $t^{\prime}$ for generic $\vec{f}$. 
We consider the cell $G r\left(t^{\prime}\right)$. Its neighborhood in $G r^{+}(\mathfrak{t})$ is diffeomorphic to $\operatorname{Gr}\left(\mathfrak{t}^{\prime}\right) \times W\left(\mathfrak{t}^{\prime}, \mathfrak{t}\right)$ where $W\left(\mathfrak{t}^{\prime}, \mathfrak{t}\right)$ is a neighborhood of 0 in the set

$$
\left\{V \in N_{q} G r\left(\mathfrak{t}^{\prime}\right) \mid \exp _{q}(t V) \in G r(\mathfrak{t}) \text { for } t \geq 0, t<<1 .\right\}
$$

Here $q$ is a point in $G r\left(\mathfrak{t}^{\prime}\right) . W\left(\mathfrak{t}^{\prime}, \mathfrak{t}\right)$ is a cone of the set $S W\left(\mathfrak{t}^{\prime}, \mathfrak{t}\right)$ which is an intersection of $W\left(\mathfrak{t}^{\prime}, \mathfrak{t}\right)$ with the unit sphere in $N_{q} G r\left(\mathfrak{t}^{\prime}\right)$. SW $\left(\mathfrak{t}^{\prime}, \mathfrak{t}\right)$ is of dimension $\operatorname{dim} G r(\mathfrak{t})$ $\operatorname{dim} G r\left(\mathfrak{t}^{\prime}\right)-1$.

Lemma 15.1. Let $\stackrel{\circ}{W}\left(\mathfrak{t}^{\prime}, \mathfrak{t}\right)=W\left(\mathfrak{t}^{\prime}, \mathfrak{t}\right) \cap G r(\mathfrak{t})$. Then by choosing $W\left(\mathfrak{t}^{\prime}, \mathfrak{t}\right)$ suffciently small, we have a diffeomorphism :

$$
\stackrel{\circ}{W}\left(\mathfrak{t}^{\prime}, \mathfrak{t}\right) \times \mathcal{M}_{g}\left(M: \vec{f}, \vec{p}, \mathfrak{t}^{\prime}\right) \rightarrow \mathcal{M}_{g}(M: \vec{f}, \vec{p}, \mathfrak{t}) \cap \pi^{-1}\left(W\left(\mathfrak{t}^{\prime}, \mathfrak{t}\right)\right)
$$

which is compatible to the projections to $W\left(\mathfrak{t}^{\prime}, \mathfrak{t}\right)$.

Proof. We extend the map

$$
\operatorname{Exp}_{\mathfrak{t}}: M \times G r(\mathfrak{t}) \rightarrow M^{m}
$$

defined in $\S 12$ to a continuous map :

$$
\operatorname{Exp}_{\mathfrak{t}}^{+}: M \times G r^{+}(\mathfrak{t}) \rightarrow M^{m}
$$

We regard $G r\left(\mathfrak{t}^{\prime}\right)$ as a subset of $G r^{+}(\mathfrak{t})$. Then the restriction of $\operatorname{Exp}_{\mathfrak{t}}^{+}$to $G r\left(\mathfrak{t}^{\prime}\right)$ is related to $\operatorname{Exp}_{\mathfrak{t}^{\prime}}: M \times G r\left(\mathfrak{t}^{\prime}\right) \rightarrow M^{m^{\prime}}$ as follows. We recall that $m$ is the number of interior vertices of $\mathfrak{t}$ which are contained in one of the exterior edges, and similar for $m^{\prime}$. Let $\left\{v_{1}, \cdots, v_{m}\right\} \in C_{i n t}^{0}(\mathfrak{t})$ and $\left\{v_{1}^{\prime}, \cdots, v_{m^{\prime}}^{\prime}\right\} \in C_{i n t}^{0}\left(\mathfrak{t}^{\prime}\right)$ be those vertices. Since $\mathfrak{t}^{\prime}$ is obtained by shrinking some of the edges of $\mathfrak{t}$, each of $v_{j}^{\prime}$ corresponds to some of $v_{i}$ 's. We define the map

$$
\alpha:\{1, \cdots, m\} \rightarrow\left\{1, \cdots, m^{\prime}\right\}
$$

such that $v_{\alpha(i)}^{\prime}$ is obtained from $v_{i}$. We then define $s_{\mathfrak{t}^{\prime}, t}: M^{m^{\prime}} \rightarrow M^{m}$ by

$$
s_{\mathfrak{t}^{\prime}, \mathfrak{t}}\left(\left(p_{j}\right)_{1 \leq j \leq m^{\prime}}\right)=\left(q_{i}\right)_{1 \leq i \leq m} \quad \text { where } q_{i}=p_{j} \text { for } j=\alpha(i) .
$$

Then by definition the map

$$
s_{\mathfrak{t}^{\prime}, \mathfrak{t}} \circ \operatorname{Exp}_{\mathfrak{t}^{\prime}}: M \times G r\left(\mathfrak{t}^{\prime}\right) \rightarrow M^{m}
$$

coincides with the restriction of $E x p_{\mathfrak{t}}^{+}$. Therefore by using (12.1.2) for $\mathfrak{t}^{\prime}$, we can prove that restriction of $E x p_{t}^{+}$to $M \times G r\left(t^{\prime}\right)$ is transversal to

$$
\prod_{h=1}^{m} \bigcap_{j=i_{h}}^{i_{h+1}-1} W_{p_{j}}^{-}\left(f_{j+1}-f_{j}\right)
$$

Lemma 15.1 then immediately follows from the implicit function theorem.

Now we are ready to prove Theorem 1.6. For each $\mathfrak{t}$, we consider

$$
\mathcal{M}_{g}^{+}(M: \vec{f}, \vec{p}, \mathfrak{t})=\mathcal{M}_{g}(M: \vec{f}, \vec{p}, \mathfrak{t}) \cup \coprod_{\mathfrak{t} \succ \mathfrak{t}^{\prime}} \mathcal{M}_{g}\left(M: \vec{f}, \vec{p}, \mathfrak{t}^{\prime}\right)
$$

We define a topology and a smooth structure on it so that the map

$$
W\left(\mathfrak{t}^{\prime}, \mathfrak{t}\right) \times \mathcal{M}_{g}\left(M: \vec{f}, \vec{p}, \mathfrak{t}^{\prime}\right) \rightarrow \mathcal{M}_{g}^{+}(M: \vec{f}, \vec{p}, \mathfrak{t})
$$


in Lemma 15.1, gives a coordinate chart. Then by Lemma 15.1 the space $\mathcal{M}_{g}^{+}(M$ : $\vec{f}, \vec{p}, \mathfrak{t})$ becomes a smooth manifold with corners. For $\mathfrak{t} \succ \mathfrak{t}^{\prime}$, there is a natural inclusion

$$
\mathcal{M}_{g}^{+}\left(M: \vec{f}, \vec{p}, \mathfrak{t}^{\prime}\right) \subset \mathcal{M}_{g}^{+}(M: \vec{f}, \vec{p}, \mathfrak{t})
$$

Using these inclusions we can patch $\mathcal{M}_{g}^{+}(M: \vec{f}, \vec{p}, \mathfrak{t})$ 's together to provide a topology on $\mathcal{M}_{g}(M: \vec{f}, \vec{p})$. It remains to define a smooth structure on it.

Let $u \in \mathcal{M}_{g}(M: \vec{f}, \vec{p}, t) \subset \mathcal{M}_{g}(M: \vec{f}, \vec{p})$. Then its neighborhood in $\mathcal{M}_{g}(M: \vec{f}, \vec{p})$ is the union of

$$
\bigcup_{\mathfrak{t}^{\prime} ; \mathfrak{t} \succ \mathfrak{t}^{\prime}} W\left(\mathfrak{t}^{\prime}, \mathfrak{t}\right) \times U
$$

where $U$ is a neighborhood of $u$ in $\mathcal{M}_{g}(M: \vec{f}, \vec{p}, \mathfrak{t})$. We remark that for a given $\mathfrak{t}$ the set

$$
\bigcup_{\mathfrak{t}^{\prime} ; \mathfrak{t} \succ \mathfrak{t}^{\prime}} W\left(\mathfrak{t}^{\prime}, \mathfrak{t}\right)
$$

is identified with a neighborhood of 0 in the fibre of the normal bundle of $G r(\mathfrak{t})$ in $G r_{k}$. Since we have already proved that $G r_{k}$ is a smooth manifold, it follows that $\bigcup_{\mathfrak{t}^{\prime} ; \mathfrak{t} \succ \mathfrak{t}^{\prime}} W\left(\mathfrak{t}^{\prime}, \mathfrak{t}\right)$ becomes a smooth manifold. We thus get a chart of $\mathcal{M}_{g}(M: \vec{f}, \vec{p})$ in a neighborhood of $u$. It is obvious that these charts are compatible and so define a smooth structure of $\mathcal{M}_{g}(M: \vec{f}, \vec{p})$.

One can find the dimension of $\mathcal{M}_{g}(M: \vec{f}, \vec{p})$ by using Lemma 12.4. The smoothness of the projection

$$
\mathcal{M}_{g}(M: \vec{f}, \vec{p}) \rightarrow G r_{k}
$$

is also obvious from the construction. Hence the proof of Theorem 1.6.

16. Behavior of holomorphic disks in $\mathbb{C}^{n}$. To complete the proof of our main theorem, one might try to glue the locally defined diffeomorphisms in $\S 13$. However, it is more efficient to glue the local approximate solutions $\Psi_{\mathrm{t}: \alpha}^{\epsilon}$ and produce global approximate solutions and then to apply the perturbation method used in Part I once and for all, than to glue the diffeomorphisms $\Xi_{t: \alpha}^{\epsilon}$. For this purpose, we will further study the properties of the maps $w_{\Theta(\ell)}$ obtained via Lemma 11.2.

Let $\mathfrak{t} \in G_{k}, \ell \in G r(\mathfrak{t})$. The space $\Theta(\ell)$ contains $k$ ends $\Theta_{i}(\ell), i=1, \cdots, k$, each of which is isometric to $(-\infty, 0] \times[0,1]$. Let $a_{i} \in \mathbb{R}^{n}$. We use the same notation as in Proposition 11.1.

We consider the graph $T$ with metric $\ell$, and remark that there is an isometric embedding of $T$ to $\Theta(\ell)$, such that each vertex of $T$ is mapped to a singular point (with respect to the flat metric mentioned before) of $\Theta(\ell)$. Hereafter we will regard $T$ as a subset of $\Theta(\ell)$. Let $v_{1}, v_{2} \in C_{i n t}^{0}(t)$. We denote by $\overline{v_{1} v_{2}}$ the minimal path joining them in $T$. Let $e_{j_{1}}, \cdots, e_{j_{m}} \in C_{i n t}^{1}(\mathfrak{t})$ be the edges contained in this path in this order. We put

$$
e v\left(v_{2}, v_{1}, \ell,\left(a_{i}\right)\right)=\sum_{i} \ell\left(e_{j_{i}}\right) a_{j_{i}} .
$$

We extend the map ev as follows. Let $\theta_{1}, \theta_{2} \in T$. Suppose that $\theta_{i}$ is on the edge $e_{j(i)}$. We consider the case when $e_{j(i)}$ are interior edges $(i=1,2)$. Let $s_{i} \in\left[0, \ell_{j(i)}\right]$ be the point corresponding to $\theta_{i}$ and $v_{i}$ be the vertex corresponding to $0 \in\left[0, \ell_{j(i)}\right]$. We put

$$
e v\left(\theta_{1}, \theta_{2}, \ell,\left(a_{i}\right)\right)=e v\left(v_{2}, v_{1}, \ell,\left(a_{i}\right)\right)+s_{2} a_{j(2)}+\left(\ell\left(e_{j(1)}\right)-s_{1}\right) a_{j(1)} .
$$


The definition in the case when $e_{j(i)}$ is exterior is similar. It is easy to see that $\theta \mapsto e v\left(\theta, \theta_{2}, \ell,\left(a_{i}\right)\right)$ is a PL map. We remark that this map is a special case of the map Exp used in $\S 12$. (Namely this corresponds to the case when we choose $f_{i}$ to be the quadratic function whose gradient vector fields are constant vector fields $a_{i}$.)

Proposition 16.1. Let $\widetilde{w}_{\Theta(\ell)}$ be the map defined in Proposition 11.1 on $\Theta(\ell)$. Then there exists a constant $C_{1}$ independent of $\ell$ such that

$$
\left|\tilde{w}_{\Theta(\ell)}\left(\theta_{2}\right)-\tilde{w}_{\Theta(\ell)}\left(\theta_{1}\right)-e v\left(\theta_{2}, \theta_{1}, \ell,\left(a_{i}\right)\right)\right|<C_{1}
$$

Proof. We will prove Proposition 16.1 together with Lemma 11.2 simultaneously. We first prove the following.

LEMMA 16.2. Let $\mathfrak{t} \succ \mathfrak{t}^{\prime}$. For each positive constant $C_{2}$, there exists constants $0<C_{3}=C_{3}\left(C_{2}, \mathfrak{t}\right)$ and $0<C_{4}=C_{4}\left(C_{2}, \mathfrak{t}\right)$ depending only on $\mathfrak{t}, C_{2}$, such that the following holds: If $\ell \in G r(\mathfrak{t})$ and satisfies

$$
\begin{aligned}
& \ell(e)>C_{3}, \quad e \in C_{\text {int }}^{1}(\mathfrak{t})-C_{\text {int }}^{1}\left(\mathfrak{t}^{\prime}\right) \\
& \ell(e)<C_{2}, \quad e \in C_{\text {int }}^{1}\left(\mathfrak{t}^{\prime}\right) \subset C_{\text {int }}^{1}(\mathfrak{t})
\end{aligned}
$$

then the conclusion of Proposition 16.1 holds for $C_{1}=C_{4}$ and Lemma 11.2 holds for such $\mathbf{t}$.

Postponing the proof of this lemma to the end of this section, we first prove that Lemma 16.2 implies Proposition 16.1. (This is rather a technical part of the proof.) Let

$$
\begin{aligned}
& C_{3}\left(C_{2}, n, m\right)=\sup \left\{C_{3}\left(C_{2}, \mathfrak{t}\right) \mid \sharp C_{i n t}^{0}(\mathfrak{t})=n, \sharp C_{i n t}^{0}\left(\mathfrak{t}^{\prime}\right)=m\right\} \\
& C_{4}\left(C_{2}, n, m\right)=\sup \left\{C_{4}\left(C_{2}, \mathfrak{t}\right) \mid \sharp C_{\text {int }}^{0}(\mathfrak{t})=n, \sharp C_{\text {int }}^{0}\left(\mathfrak{t}^{\prime}\right)=m\right\}
\end{aligned}
$$

We put $B_{1}=C_{3}(1, k, 1), B_{1}^{\prime}=C_{4}(1, k, 1)$. Suppose $\ell(e)>B_{1}$ for each $e \in C_{i n t}^{1}(\mathfrak{t})$. In this case, Lemma 16.2 implies that Proposition 16.1 holds for such $\ell$ with $C_{1}=B_{1}^{\prime}$. (We remark that if $\sharp C_{\text {int }}^{0}\left(\mathrm{t}^{\prime}\right)=1$ then $C_{\text {int }}^{1}\left(\mathrm{t}^{\prime}\right)$ is empty.)

We next consider the case where $\ell(e) \leq B_{1}$ for some $e$. We collapse all such edges to obtain $\mathfrak{t}^{\prime}$. If we also assume that

$$
\ell(e)>C_{3}\left(B_{1}, \mathfrak{t}\right)
$$

for each edge $e \in C_{i n t}^{1}(\mathfrak{t})-C_{i n t}^{1}\left(\mathfrak{t}^{\prime}\right)$, then we can apply Lemma 16.2 and derive that Proposition 16.1 holds with $C_{1}=C_{4}\left(B_{1}, \mathfrak{t}\right)$.

In other words, we have proved the following: Let $B_{2}=\sup _{m} C_{3}\left(B_{1}, k, m\right), B_{2}^{\prime}=$ $\sup _{m} C_{4}\left(B_{1}, k, m\right)$. If Proposition 16.1 is false for $C_{1}=C_{4}\left(B_{1}, \mathfrak{t}, \mathrm{t}^{\prime}\right)$, then there exists $e \in C_{\text {int }}^{1}(\mathfrak{t})-C_{\text {int }}^{1}\left(\mathfrak{t}^{\prime}\right)$ such that $\ell(e)<B_{2}$. It follows in particular that there exists at least two edges $e$ in $t$ such $\ell(e)<B_{2}$.

We repeat this procedure inductively and can prove the following: Define $B_{u+1}=$ $\sup _{m} C_{3}\left(B_{u}, k, m\right), B_{u+1}^{\prime}=\sup _{m} C_{4}\left(B_{u}, k, m\right)$. If Proposition 16.1 is false with $C_{1}=$ $B_{u+1}^{\prime}$, then there exists at least $u+1$ edges $e$ such that $\ell(e)<B_{u+1}$. On the other hand, the number of edges is smaller than $k-3$. Therefore Proposition 16.1 holds for $C_{1}=\sup _{j \leq k-2} B_{j}^{\prime}$.

Now it remains to prove Lemma 16.2. Let $v \in C_{i n t}^{0}\left(\mathfrak{t}^{\prime}\right)$. We put $\overline{\mathfrak{c}}_{v}=\pi^{-1}(v) \subset \mathfrak{t}$ and define $\mathfrak{c}_{v}$ as in $\S 10$. Restricting $\ell: C_{\text {int }}^{1}(\mathfrak{t}) \rightarrow \mathbb{R}_{+}$to $\mathfrak{c}_{v}$, we obtain $\ell_{v} \in \operatorname{Gr}\left(\mathfrak{c}_{v}\right)$. Using Lemma 11.1, we obtain

$$
\widetilde{w}_{v}: \Theta\left(\ell_{v}\right) \rightarrow \mathbb{C}^{n}
$$


(which will be normalized as in (17.1)). For each $e \in C^{1}\left(\mathfrak{t}^{\prime}\right)$ with $v \in \partial e$, we have a subset in $\Theta\left(\ell_{v}\right)$

$$
\Theta_{e}\left(\ell_{v}\right) \simeq(-\infty, 0] \times[0,1] \subset \Theta\left(\ell_{v}\right) .
$$

Using the coordinates $(\tau, t)$ there, we have

$$
\mid \widetilde{w}_{v}(\tau, t)+\text { const }_{e}-\left(\tau\left(a_{\text {leg }(e)}-a_{\text {rig }(e)}\right)+\sqrt{-1} t\left(a_{l e g(e)}-a_{\text {rig }(e)}\right) \mid<o(\tau)\right.
$$

Here $o(\tau)$ is a function going to 0 as $\tau$ goes to infinity. We remark that we can choose $o(\tau)$ depending only on $C_{2}$ and $a_{i}$. This is a consequence of the fact that $\Theta\left(\ell_{v}\right)$ from its definition consists of a compact family because we imposed that $\ell(e) \leq C_{2}$ for all $e \in C_{\text {int }}^{1}\left(\mathfrak{c}_{v}\right)$. by

For $e \in C^{1}\left(\mathfrak{t}^{\prime}\right)$ we take rectangles $[0, \ell(e)] \times[0,1]$ or $(-\infty, 0] \times[0,1]$ and define $\widetilde{w}_{e}$

$$
\widetilde{w}_{e}(\tau, t)=\tau\left(a_{l e g(e)}-a_{\text {rig }(e)}\right)+\sqrt{-1}\left(t\left(a_{l e g(e)}-a_{\text {rig }(e)}\right)+a_{\text {rig }(e)}\right)
$$

We take $\widetilde{w}_{v}+$ const $_{v}$ and $\widetilde{w}_{e}+$ const $_{e}$ with appropriate constants and glue them in a way similar to $\S 11$ along the intermediate rectangles shown as in (16.2.3) below for a sufficiently large constant $C_{3}$ which be chosen later. We then obtain a map

$$
w_{0}: \Theta(\ell) \rightarrow \mathbb{C}^{n} .
$$

By construction, it will have the following properties:

(16.2.1) $w_{0}\left(\partial_{i}(\Theta(\ell))\right) \subset \Lambda_{i}$.

(16.2.2) $\left|w_{0}\left(\theta_{2}\right)-w_{\Theta(\ell)}\left(\theta_{1}\right)-e v\left(\theta_{2}, \theta_{1}, \ell,\left(a_{i}\right)\right)\right|<C_{5}\left(C_{2}, \mathfrak{t}, \mathfrak{t}^{\prime}\right)$

(16.2.3) $\bar{\partial} w_{0}$ is supported on

$$
\begin{gathered}
\left(\bigcup_{e \in C_{i n t}^{1}\left(\mathfrak{t}^{\prime}\right)}\left(\left[2 C_{3} / 7,3 C_{3} / 7\right] \cup\left[\ell(e)-3 C_{3} / 7, \ell(e)-2 C_{3} / 7\right]\right) \times[0,1]\right) \\
\cup\left(\bigcup_{e \in C_{e x t}^{1}\left(\mathfrak{t}^{\prime}\right)}\left[-3 C_{3} / 7,-2 C_{3} / 7\right] \times[0,1]\right)
\end{gathered}
$$

(16.2.4) $\left|\sup \bar{\partial} w_{0}\right|<o(\tau)$

(16.2.5) (11.1.2) holds for $w_{0}$ uniformly over $\ell$ that satisfies (16.1.1) and (16.1.2).

Therefore to complete the proof of Lemma 16.2, it will be enough to prove the following:

LEMMA 16.3. There exists $h_{\ell}: \Theta(\ell) \rightarrow \mathbb{C}$ such that $\bar{\partial} h_{\ell}=\bar{\partial} w_{0}, h_{\ell}$ is bounded, $\left.h_{\ell}\right|_{\partial \Theta(\ell)}$ is real valued, and $h_{\ell}$ goes to zero uniformly over $\ell$ at their ends, provided $\ell$ satisfies (16.1) for a sufficiently large $C_{3}=C_{3}\left(C_{2}, \mathfrak{t}, \mathfrak{t}^{\prime}\right)$.

Proof. We prove Lemma 16.3 by iteration. First we solve

$$
\left\{\begin{array}{c}
\bar{\partial} h_{1}^{e}=\bar{\partial} w_{0} \\
h_{1}^{e}(0, t)=h_{1}^{e}(\ell(e), t)=0 \\
h_{1}^{e}(\tau, 0), h^{e}(\tau, 1) \in \mathbb{R}^{n}
\end{array}\right.
$$

on the rectangle $\Theta_{e}(\ell)=[0, \ell(e)] \times[0,1]$ for each $e \in C_{i n t}^{1}\left(\mathfrak{t}^{\prime}\right)$ and on $\Theta_{e}(\ell)=(-\infty, 0] \times$ $[0,1]$ for each $e \in C_{e x t}^{1}\left(\mathfrak{t}^{\prime}\right)$. Note that this equation has the unique solution $h_{1}^{e}=$ $G^{e}\left(\bar{\partial} w_{0}\right)$ where $G^{e}$ is the Green's operator for the rectangle $\Theta_{e}(\ell)$. Then by using 
(16.2.3) and some elementary properties of the Green function of the rectangle, we have, for each $e \in C_{\text {int }}^{1}\left(\mathfrak{t}^{\prime}\right)$

$$
\begin{gathered}
\left|h_{1}^{e}\right|_{\left[0, C_{3} / 7\right] \times[0,1]} \mid<a e^{-b C_{3}} . \\
\left|h_{1}^{e}\right|_{\left[\ell(e)-C_{3} / 7, \ell(e)\right] \times[0,1]} \mid<a e^{-b C_{3}} .
\end{gathered}
$$

for positive constants $a, b$. We obtain similar inequalities for $e \in C_{e x t}^{1}\left(\mathfrak{t}^{\prime}\right)$ considering $\left[-C_{3} / 7,0\right]$.

We then take a cut-off function $\chi_{e}$ such that

$$
\chi_{e}(\tau)= \begin{cases}0 & \text { in a neighborhood of } 0 \text { and } \ell(e) \\ 1 & \text { for } \tau \in\left[C_{3} / 7, \ell(e)-C_{3} / 7\right]\end{cases}
$$

for $e \in C_{\text {int }}^{1}\left(\mathfrak{t}^{\prime}\right)$ and similarly for each $e \in C_{e x t}^{1}\left(\mathfrak{t}^{\prime}\right)$. We put

$$
w_{0}^{\prime}=w_{0}-\sum \chi_{e} h_{1}^{e} \text {. }
$$

It follows that $w_{0}^{\prime}$ has the following properties:

(16.3.1) $\bar{\partial} w_{0}^{\prime}$ is supported on

$$
\bigcup_{e \in C_{i n t}^{1}\left(t^{\prime}\right)}\left[0, C_{3} / 7\right] \cup\left[\ell(e)-C_{3} / 7, \ell(e)\right] \times[0,1] \cup \bigcup_{e \in C_{e x t}^{1}\left(t^{\prime}\right)}\left[-C_{3} / 7,0\right] \times[0,1]
$$

(16.3.2) $\sup \left|\bar{\partial} w_{0}^{\prime}\right|<a e^{-b C_{3}}$.

We next consider

$$
\Theta^{\prime}\left(\ell_{v}\right)=\Theta\left(\ell_{v}\right)-\bigcup_{e \in C_{e x t}^{1}\left(\mathfrak{c}_{v}\right)}\left(-\infty,-3 C_{3} / 7\right] \times[0,1]
$$

and solve the equation there

$$
\left\{\begin{aligned}
\bar{\partial} h_{2}^{v} & =\bar{\partial} w_{0}^{\prime} \\
\left.h_{2}^{v}\right|_{\left\{-3 C_{3} / 7\right\} \times[0,1]} & =0 \\
\left.h_{2}^{v}\right|_{\partial \Theta\left(\ell_{v}\right) \cap \Theta^{\prime}\left(\ell_{v}\right)} & \in \mathbb{R}^{n}
\end{aligned}\right.
$$

Again the unique solution of this is given by

$$
h_{2}^{v}=G_{C_{3}}^{v}\left(\bar{\partial} w_{0}^{\prime}\right)=G_{C_{3}}^{v}\left(\bar{\partial}\left(w_{0}-\sum_{e} \chi_{e} h_{1}^{e}\right)\right)
$$

where $G_{C_{3}}^{v}$ is the Green's operator of $\Theta^{\prime}\left(\ell_{v}\right)$. Using an estimate of the Green function on $\Theta^{\prime}\left(\ell_{v}\right)$, we find that

$$
\sup \left|h_{2}^{v}\right| \leq C C_{3}^{N} \sup \left|\bar{\partial} w_{0}^{\prime}\right|
$$

for some constants $C$ and $N$. We take $\chi_{e}^{\prime}$ for each $e \in C_{e x t}^{1}\left(\mathfrak{c}_{v}\right)$ such that

$$
\chi_{e}^{\prime}(\tau)= \begin{cases}0 & \text { in a neighborhood of }-3 C_{3} / 7 \\ 1 & \tau \in\left[-C_{3} / 7,0\right]\end{cases}
$$

We extend this to $\Theta^{\prime}\left(\ell_{v}\right)$ by setting to be equal to 1 at the point corresponding to the vertex $v$ and denote by $\chi_{v}^{\prime}$ the function so obtained. We put

$$
w_{1}=w_{0}^{\prime}-\sum \chi_{v}^{\prime} h_{2}^{v}
$$


We can repeat the construction above by using $w_{1}$ in place of $w_{0}$ and obtain $w_{2}, w_{3}$, $w_{4}, \cdots$. These will satisfy the recursion forumla

$$
w_{n+1}=w_{n}-\sum_{e} \chi_{e} G^{e}\left(\bar{\partial} w_{n}\right)-\sum_{v} \chi_{v}^{\prime} G_{C_{3}}^{v}\left(\bar{\partial}\left(w_{n}-\sum_{e} \chi_{e} G^{e}\left(\bar{\partial} w_{n}\right)\right)\right)
$$

for $n \geq 0$. We remark that $a C C_{3}^{N} e^{-b C_{3}}<<1$, if $C_{3}$ is sufficiently large. In this case, one can easily check from (16.4) that $w_{i}$ converges to $w_{\infty}$ as $i \rightarrow \infty$, that $\bar{\partial} w_{\infty}=0$ and that $h=w_{0}-w_{\infty}$ satisfies the required properties.

The proof of Lemma 16.2 and hence of Proposition 16.1 is now complete. Furthermore we have also finished the proof of Lemma 11.2 noting that both $\widetilde{w}_{\Theta(\ell)}$ and $w_{\infty}$ satisfy (11.1.1) and (11.1.2) and so the difference $\widetilde{w}_{\Theta(\ell)}-w_{\infty}$ is constant by the uniqueness statement in Proposition 11.1.

17. Gluing diffeomorphisms. Now we come to the final step of the proof of our main theorem. We need to modify our map $\Psi_{\mathrm{t}, \alpha}^{\epsilon}$ in order to glue them. Suppose $\mathfrak{t} \succ \mathfrak{t}^{\prime}$. Then for $v \in C_{\text {int }}^{0}\left(\mathfrak{t}^{\prime}\right)$, the trees $\mathfrak{c}_{v}(\mathfrak{t}), \overline{\mathfrak{c}}_{v}(\mathfrak{t})$ are defined as in Definition 10.5. For each $v \in C_{\text {int }}^{0}(\mathfrak{t})$ and $\ell \in U\left(\mathfrak{t}^{\prime} ; \epsilon, \alpha\right) \cap G r(\mathfrak{t})$, we fix a point

$$
\theta\left(\mathfrak{c}_{v}(\mathfrak{t}), \ell_{v}\right) \in \overline{\mathfrak{c}}_{v}(\mathfrak{t})
$$

such that, if $\left(\mathfrak{t}_{i}, \ell_{v_{i}}\right)$ converges to $\left(\mathfrak{t}, \ell_{v}\right)$ then $\theta\left(\mathfrak{c}_{v}\left(\mathfrak{t}_{i}\right), \ell_{v_{i}}\right)$ converges to $\theta\left(\mathfrak{c}_{v}(\mathfrak{t}), \ell_{v}\right)$. Now we are going to construct

$$
\Psi_{\mathfrak{t}^{\prime}, \alpha}^{\prime, \epsilon}: U\left(\mathfrak{t}^{\prime}: \epsilon, \alpha\left(\mathfrak{t}^{\prime}\right)\right) \times_{\pi_{\epsilon}} \mathcal{M}_{g}(M: \vec{f}, \vec{p}) \rightarrow \operatorname{Map}\left(T^{*} M: \vec{\Lambda}^{\epsilon}, \vec{p}^{\epsilon}\right)
$$

Let $\ell \in U\left(\mathfrak{t}^{\prime}: \epsilon, \alpha\right) \cap G r(\mathfrak{t}), I \in \mathcal{M}_{g}(M: \vec{f}, \vec{p})$ such that $\pi_{\epsilon}(\ell)=\pi(I)$. For each $v \in C_{\text {int }}^{0}\left(\mathfrak{t}^{\prime}\right)$, we have $\theta\left(\mathfrak{c}_{v}(\mathfrak{t}), \ell_{v}\right) \in \overline{\mathfrak{c}}_{v} \subset \mathfrak{t}$. We put

$$
p_{v}=I\left(\theta\left(\mathfrak{c}_{v}(\mathfrak{t}), \ell_{v}\right)\right) \in M .
$$

By Lemma 11.2, we find

$$
\widetilde{w}_{v}: \Theta\left(\ell_{v}\right) \rightarrow \mathbb{C}^{n} \cong T_{\left(p_{v}, 0\right)}\left(T^{*} M\right),
$$

such that

$$
\operatorname{Re} \widetilde{w}_{v}\left(\theta\left(\mathfrak{c}_{v}(\mathfrak{t}), \ell_{v}\right)\right)=0 .
$$

We use (17.1) to remove the ambiguity in Lemma 11.2 (or Proposition 11.1).

Let $\theta \in \mathfrak{c}_{v}(\mathfrak{t}) \cap \mathfrak{t}$. (We identify a part $[-\ell(e), 0]$ of the exterior edge $e$ of $\mathfrak{c}_{v}$ to the corresponding edge of $\mathfrak{t}^{\prime}$ that contains $v$.)

Lemma 17.1. For any $\theta \in \mathfrak{c}_{v}(\mathfrak{t}) \cap \mathfrak{t}\left(\subset \Theta_{v}\right)$, there exists some constant $C>0$ such that

$$
\operatorname{dist}\left(\exp _{p_{v}}\left(\epsilon \widetilde{w}_{v}(\theta)\right), I(\theta)\right)<C \max \left\{\epsilon, \epsilon^{2(1-\alpha)}\right\} .
$$

(We remark that $\theta \in \mathfrak{c}_{v} \subset \Theta_{v}$ and also $\theta \in \mathfrak{t}$ by our construction.)

Proof. Lemma 17.1 will be a consequence of Proposition 16.1. Let $e$ an edge containing $\theta\left(\mathfrak{c}_{v}(\mathfrak{t}), \ell_{v}\right)$. We identify $e=[0, \ell(e)]$ where 0 corresponds to a vertex $v_{1}$ and $\ell(e)$ corresponds to another vertex $v_{2}$. Let $s \in[0, \ell(e)] \simeq e$ be the coordinate of the point $\theta\left(\mathfrak{c}_{v}(\mathfrak{t}), \ell_{v}\right)$ in $e$. By Proposition 16.1, we have

$$
\left|\widetilde{w}_{v}(\theta)-\widetilde{w}_{v}\left(\theta\left(\mathfrak{c}_{v}(\mathfrak{t}), \ell_{v}\right)\right)-e v\left(\theta, \theta\left(\mathfrak{c}_{v}(\mathfrak{t}), \ell_{v}\right), \ell_{v} ;\left(-\operatorname{grad} f_{j(i)}\right)\right)\right|<C_{1}
$$


On the other hand, it follows from (11.4) that

$$
\mid \operatorname{Im} \widetilde{w}_{v}\left(\theta\left(\mathfrak{c}_{v}(\mathfrak{t}), \ell_{v}\right) \mid<C\right.
$$

uniformly over $\ell_{v}$. Combining this with the normalization condition (17.1), we have

$$
\mid \widetilde{w}\left(\theta\left(\mathfrak{c}_{v}(\mathfrak{t}), \ell_{v}\right) \mid<C .\right.
$$

This together with (17.2) implies

$$
\left|\widetilde{w}_{v}(\theta)-e v\left(\theta, \theta\left(\mathfrak{c}_{v}(\mathfrak{t}), \ell_{v}\right), \ell_{v} ;\left(-\operatorname{grad} f_{j(i)}\right)\right)\right|<C .
$$

Now let $e_{j_{1}}, \cdots, e_{j_{m}} \in C_{i n t}^{1}\left(\boldsymbol{c}_{v}\right)$ be the edges joining $v_{2}$ to $\theta$ in this order. And let $\theta_{2}$ be the point in $e_{j_{m}}$ corresponding to $s^{\prime} \in\left[0, \ell\left(e_{j_{m}}\right)\right]$. By definition

$$
\begin{aligned}
I(\theta) & =\exp \left(-\epsilon s^{\prime}\left(f_{l e g\left(e_{j_{m}}\right)}-f_{\text {rig }\left(e_{j_{m}}\right)}\right) \circ \exp \left(-\epsilon \ell\left(e_{j_{m-1}}\right)\left(f_{l e g\left(e_{j_{m-1}}\right)}-f_{\text {rig }\left(e_{j_{m-1}}\right)}\right) \circ \cdots\right.\right. \\
& \circ \exp \left(-\epsilon \ell\left(e_{j_{1}}\right)\left(f_{l e g\left(e_{j_{1}}\right)}-f_{r i g\left(e_{j_{1}}\right)}\right) \circ \exp \left(-\epsilon(\ell(e)-s)\left(f_{l e g(e)}-f_{r i g(e)}\right)\right)\left(p_{v}\right)\right.
\end{aligned}
$$

We remark that

$$
\operatorname{dist}\left(\exp \left(V_{1}\right) \circ \cdots \circ \exp \left(V_{n}\right)(p), \exp \left(V_{1}+\cdots+V_{n}\right)(p)\right)<C\left(\left|V_{1}\right|+\cdots+\left|V_{n}\right|\right)^{2}
$$

for vector fields $V_{i}$, and recall that $\ell(e)<\epsilon^{-\alpha}$. Therefore we have

$$
\operatorname{dist}\left(\exp _{p_{v}}\left(\epsilon \operatorname{ev}\left(\theta, \theta\left(\mathfrak{c}_{v}(\mathfrak{t}), \ell_{v}\right), \ell_{v} ;\left(-\operatorname{grad} f_{j(i)}\right)\right), I(\theta)\right)<C \epsilon^{2(1-\alpha)} .\right.
$$

On the other hand note that if $\left|\xi_{1}-\xi_{2}\right|<C$ for $\xi_{i} \in T_{\left(p_{v}, 0\right)}\left(T^{*} M\right) \cong \mathbb{C}^{n}$, then

$$
\operatorname{dist}\left(\exp _{p_{v}} \epsilon \xi_{1}, \exp _{p_{v}} \epsilon \xi_{2}\right)<C \epsilon .
$$

It follows by using (17.3) and applying this inequality to

$$
\xi_{1}=\widetilde{w}_{v}(\theta), \quad \xi_{2}=e v\left(\theta, \theta\left(\mathfrak{c}_{v}(\mathfrak{t}), \ell_{v}\right), \ell_{v} ;\left(-\operatorname{grad} f_{j(i)}\right)\right)
$$

that we have

$$
\operatorname{dist}\left(\exp _{p_{v}} \epsilon\left(\widetilde{w}_{v}(\theta)\right), \quad \exp _{p_{v}}\left(\epsilon e v\left(\theta, \theta\left(\mathfrak{c}_{v}(\mathfrak{t}), \ell_{v}\right), \ell_{v} ;\left(-\operatorname{grad} f_{j(i)}\right)\right)\right)<C \epsilon\right.
$$

Combining (17.4) and (17.5), we have finished the proof.

We remark that Lemma 11.5 is an immediate consequence of Lemma 17.1.

Using Lemma 17.1, we glue $\widetilde{w}_{v}$ and maps obtained from $\left.I\right|_{e}\left(e \in C^{1}(\mathfrak{t})\right)$, in the same way as in the proof of Proposition 11.4 and obtain

$$
\Psi_{\mathfrak{t}^{\prime}, \alpha}^{\prime, \epsilon}: U\left(\mathfrak{t}^{\prime}: \epsilon, \alpha\left(\mathfrak{t}^{\prime}\right)\right) \times_{\pi_{\epsilon}} \mathcal{M}_{g}(M: \vec{f}, \vec{p}) \rightarrow \operatorname{Map}\left(M: \vec{\Lambda}^{\epsilon}, \vec{p}^{\epsilon}\right) .
$$

Now using Lemma 17.1 again, we repeat the proof of $\S 5$ to show the following :

LEMMA 17.2. Let $\ell \in U\left(\mathfrak{t}_{1}: \epsilon, \alpha\left(\mathfrak{t}_{1}\right)\right) \cap U\left(\mathfrak{t}_{2}: \epsilon, \alpha\left(\mathfrak{t}_{2}\right)\right), I \in \mathcal{M}_{g}(M: \vec{f}, \vec{p})$ such that $\pi_{\epsilon}(\ell)=\pi(I)$. We have $\mathfrak{t}_{1} \succ \mathfrak{t}_{2}$ or $\mathfrak{t}_{2} \succ \mathfrak{t}_{1}$. Suppose $\mathfrak{t}_{1} \succ \mathfrak{t}_{2}$. We then have

$$
\sup \left(\operatorname{dist}\left(\Psi_{\mathfrak{t}_{1}, \alpha\left(\mathfrak{t}_{1}\right)}^{\prime, \epsilon}(\ell, I)(z), \Psi_{\mathfrak{t}_{2}, \alpha\left(\mathfrak{t}_{2}\right)}^{\prime, \epsilon}(\ell, I)(z)\right)\right)<C \max \left\{\epsilon, \epsilon^{2\left(1-\alpha\left(\mathfrak{t}_{2}\right)\right)}\right\} .
$$

for $z \in \Theta(\ell)$.

We next glue these maps using the technique of center of mass. We choose a partition of unity $\left\{\chi_{\mathfrak{t}}\right\}_{\mathfrak{t} \in G_{k}}$ of $G r_{k}$ subordinate to the covering $\{U(\mathfrak{t}: \epsilon, \alpha(\mathfrak{t}))\}_{\mathfrak{t} \in G_{k}}$. Let $(\ell, I) \in G r_{k} \times_{\pi_{\epsilon}} \mathcal{M}_{g}(M: \vec{f}, \vec{p})$ and define

$$
\widetilde{\Phi}^{\epsilon}(\ell, I): \Theta\left(\frac{\ell}{\epsilon}\right) \times T^{*} M \rightarrow \mathbb{R}
$$


by

$$
\tilde{\Phi}^{\epsilon}(\ell, I)(z, x)=\sum_{t} \chi_{\mathfrak{t}} \operatorname{dist}\left(\Psi_{\mathfrak{t}, \alpha(\mathrm{t})}^{\prime, \epsilon}(\ell, I)(z), x\right) .
$$

Using Lemma 17.2 and the standard results on center of mass technique (e.g., see [GK]), the map $x \mapsto \widetilde{\Phi}^{\epsilon}(\ell, I)(z, x)$ has a unique minimum for each $(\ell, I)$ and $z$ for each given $x \in T^{*} M$, which we denote

$$
\widetilde{\Phi}^{\epsilon}(\ell, I)(z) \in T^{*} M .
$$

Thus we have defined :

$$
\widetilde{\Phi}^{\epsilon}: G r_{k} \times_{\pi_{\epsilon}} \mathcal{M}_{g}(M: \vec{f}, \vec{p}) \rightarrow \operatorname{Map}\left(T^{*} M: \vec{\Lambda}^{\epsilon}, \vec{p}^{\epsilon}\right) .
$$

Proposition 17.3. If $w$ is in the image of $\tilde{\Phi}^{\epsilon}$, then we have :

$$
\left\|\bar{\partial}_{J} w\right\|_{0, p, \epsilon} \leq C \epsilon^{\frac{2+(p-1) \alpha}{p}} .
$$

Once we have proved Lemma 17.2, the proof of Proposition 17.3 is similar to that of Proposition 5.2 and so we omit the details.

We can now repeat the arguments in Part I and in $\S 13$ to perturb $\widetilde{\Phi}^{\epsilon}$ to produce a smooth map

$$
\Phi^{\epsilon}: G r_{k} \times_{\pi_{\epsilon}} \mathcal{M}_{g}(M: \vec{f}, \vec{p}) \rightarrow \mathcal{M}_{J}\left(T^{*} M: \vec{\Lambda}^{\epsilon}, \vec{p}^{\epsilon}\right) .
$$

We recall that $\pi_{\epsilon}: G r_{k} \rightarrow G r_{k}$ is a diffeomorphism. Hence $G r_{k} \times_{\pi_{\epsilon}} \mathcal{M}_{g}(M: \vec{f}, \vec{p})$ is diffeomorphic to $\mathcal{M}_{g}(M: \vec{f}, \vec{p})$. Finally we can prove in the way similar to Part I that $\Phi^{\epsilon}$ becomes a diffeomorphism for sufficiently small $\epsilon$. The proof of our main theorem is now finally complete.

\section{REFERENCES}

[Ad] J. F. AdAms, Infinite Loop Spaces, Ann. Math. Studies 90, Princeton University Press.

[AS] S. Axelrod ANd I. Singer, Chern Simons perturbation theory II, J. Diff. Geom., 39 (1994), pp. 173-213.

[Ba] D. Bar-natan, Perturbative aspects of the Chern-Simons topological field theory, PhD thesis, Princeton University, 1991.

[BC] M. BETZ AND R. Cohen, Graph moduli spaces and cohomology operations, Turkish J. Math., 18 (1995), pp. 23-41.

[Co] A. Connes, Noncommutative Geometry, Academic Press, London, 1994.

[D] S. Donaldson, A lecture at University of Warwick, 1992.

[DM] P. DELIGNE AND D. MUMFORD, The irreducibility of the space of curves of given genus, Publ. Math. IHES, 36 (1969), pp. 75-110.

[EG] Y. Eliashberg and M. Gromov, (in preparation).

[F11] A. Floer, Morse theory for Lagrangian intersections, J. Diff. Geom., 28 (1988), pp. 513-547.

[F12] - The unregularized gradient flow of the symplectic action, Comm. Pure Appl. Math., 43 (1988), pp. 576-611.

[F13] — Witten's complex and infinite dimensional Morse theory, J. Diff. Geom., 30 (1989), pp. 207-221.

[F14] — Cup length estimate for Lagrangian intersections, Comm. Pure Appl. Math., 47 (1989), pp. 335-356..

[F15] _ Symplectic fixed point and holomorphic spheres, Commun. Math. Phys., 120 (1989), pp. 575-611.

[Fu1] K. FUKAYA, Floer homology for 3-manifolds with boundary -abstract-, in Topology Geometry and Field theory, ed by Fukaya, Furuta, Khono and Kotchick, 1 - 22, World Scientific, Singapore, 1994. 
[Fu2] — Morse homotopy, $A^{\infty}$-category and Floer homologies, in Proceedings of GARC Workshop on Geometry and Topology, ed. by H. J. Kim, Seoul National University, Korea, 1993.

[Fu3] - Morse homotopy and its quantization, in AMS/IP Studies in Advanced Mathematics, International Press, 1997, pp. 409-440.

[Fu4] - Gauge theory on 4-manifolds with corners, (preprint).

[Fu5] - Morse theory and topological field theory, Suugaku Expositions, to appear.

[Fu6] - Morse homotopy and Chern-Simons perturbation theory, Commun. Math. Phys., 181 (1996), pp. 37-90.

[FM] W. Fulton And R. McPherson, A compactification of configuration space, Ann. of Math., 139 (1994), pp. 183-225.

[Ge] E. GetzleR, Operad and moduli space of genus 0 Riemann surface, (preprint).

[Gr] M. Gromov, Pseudo holomorphic curves in symplectic manifolds, Invent. Math., 82 (1985), pp. $307-347$.

[GK] K. Grove AND H. KARCher, How to conjugate $C^{1}$-close actions, Math. Z., 132 (1973), pp. $11-20$.

[GMM] E. Guadagnini, M. Martinelli, and M. Mintchev, Perturbative aspects of ChernSimons field theory, Phys. Lett. B, 227 (1989), pp. 111.

[H] H. HARER, The cohomology of the moduli space of curves, Lecture note in Math., 1337, pp. $138-221$.

[HL] Y. HUANG AND J. LePOWSKI, Vertex operator algebra and operads, (preprint).

[Ka] H. KARChER, Riemannian center of mass and mollifier smoothing, Comm. Pure and Appl. Math., 30 (1977), pp. 509-541.

[Ko1] M. Kontsevitch, Feynman diagram and low dimensional topology, in Proceedings of First European Congress of Mathematics, Birkhäuser, Boston, 1994, pp. 97-122.

[Ko2] - $A^{\infty}$-algebras in mirror symmetry, (preprint).

[KM] M. KONTSEVITCH AND Y. MANIN, Gromov-Witten classes, quantum cohomology, and enumerative geometry, 164 (1994), pp. 525-562.

[M] D. MUMForD, Toward an enumerative geometry of moduli spaces of curves, in Arithmetic and Geometry, Birkhäuser, Basel, 1983, pp. 271-326.

[MS] D. MCDUfF AND D. SAlamon, J-holomorphic Curves and Quantum Cohomology, Univ. Lec. Series, AMS, 6 (1994).

[O1] Y.-G. Он, Removal of boundary singularities of pseudo-holomorphic curves, Comm. Pure Appl. Math., 45 (1992), pp. 121-139.

[O2] - Floer cohomology of Lagrangian intersections and pseudo-holomorphic disks, I $\mathcal{G}$ II, Comm. Pure Appl. Math., 46 (1993), pp. 949-994 \& 995-1012 \& Addenda, ibid, 48, (1995), 1299-1302.

[O3] — Floer cohomology of Lagrangian intersections and pseudo-holomorphic disks, III, in Floer Memorial Volume, Birkhäuser, Basel, 1995, pp. 555-573.

[O4] - Fredholm theory of pseudo-holomorphic discs under the perturbation of boundary conditions, Math. Z., 222 (1996), pp. 505-520.

[O5] - On the structure of pseudo-holomorphic discs with totally real boundary conditions, J. Geom. Anal., to appear.

[O6] - Floer cohomology, spectral sequence and the Maslov class of Lagrangian embeddings, Intern. Math. Res. Notices, No.7 (1996), pp. 305-346.

[O7] - Relative Floer and quantum cohomology and the symplectic topology of Lagrangian submanifolds, in Newton institute proceedings for the 1994-Symplectic Topology program, C.B. Thomas, ed., Cambridge University Press, Cambridge, England.

[O8] - Symplectic topology as the geometry of action functional, I, J. Differ. Geom., to appear.

[O9] - Symplectic topology as the geometry of action functional, II, (submitted).

[P] R. PENNER, The decorated Teichmüller space of punctured surface, Commun. Math. Phys., 113 (1987), pp. 299-339.

[PSS] S. Piunikhin, D. Salamon, AND M. Schwarz, Symplectic Floer-Donaldson theory and quantum cohomology, (preprint).

[Q] D. Quillen, Rational homotopy theory, Ann. of Math., 90 (1969), pp. 205-295.

[R] Y. RUAN, Topological sigma model and Donaldson type invariant in Gromov theory, Duke Math. J., to appear.

[RT1] Y. RUAN And G. Tían, Mathematical theory of quantum cohomology, J. Differ. Geom., 42 
(1995), pp. 259-367.

[RT2] Y. RUAN AND G. TIAN, (in preparation).

[Sc] M. Schwarz, Morse homology, in Progress in Mathematics 111, Birkhäuser, Basel, 1993.

[Sm] S. SMALE, An infinite dimensional version of Sard's Theorem, Amer. J. Math., 87 (1968), pp. 861-866.

[St1] J. StASHefF, Homotopy associativity of H-spaces I, Trans. Amer. Math. Soc., 108 (1963), pp. 275-292, II, ibid. 293-312.

[St2] J. STASHeFF, Towards a closed string field theory, topological and convolution algebra, (preprint).

[Str] K. Strebel, Quadratic Differentials, Springer-Verlag, Berlin, 1985.

[Su] D. Sullivan, Infinitesimal calculations in topology, Publ. IHES, 74 (1978), pp. 269-331.

[V] C. Viterbo, Some remarks on Massey products, tied cohomology classes and LusternikSchnirelman category, (preprint) (1994).

[W1] E. Witten, Supersymmetry and Morse theory, J. Differ. Geom., 17 (1982), pp. 661-692.

[W2] - Non commutative geometry and string field theory, Nucl. Phys. B, 268 (1986), pp. 253.

[W3] - Topological sigma model, Commun. Math. Phys., 118 (1988), pp. 441.

[W4] - Chern-Simons gauge theory as a string theory, in Floer Memorial Volume, Birkhäuser, Basel, 1995, pp. 637-678. 